$$
\begin{gathered}
\text { University of Szeged } \\
\text { Faculty of Pharmacy } \\
\text { Department of Pharmaceutical Technology } \\
\text { PhD. Thesis }
\end{gathered}
$$

\title{
Development and characterization of matrix pellets prepared by extrusion and spheronization of Atenolol
}

by

\section{Elnazeer Ibrahim Mohamed Hamedelniel Pharmacist}

\author{
Supervisor \\ Prof. Dr. Klára Pintye-Hódi \\ and \\ Dr. János Bajdik
}

Szeged

2011 


\section{PUBLICATIONS}

1. Elnazeer I. Hamedelniel, János Bajdik, Klára Pintye-Hódi: Optimization of preparation of matrix pellets containing ethylcellulose, J. Chem. Eng Proc.(2009) 88, 1033-1036

2. Elnazeer I. Hamedelniel, János Bajdik, Péter Kása Jr, Klára Pintye-Hódi: Study of the influence of alkalizing components on matrix pellets prepared by extrusion/spheronization, Pharm. Dev. Techn., DOI: 10.3109/10837450.2010.531734

3. Elnazeer I. Hamedelniel, János Bajdik, Tamás Sovány, Klára Pintye-Hódi: Delayed release matrix pellet preparation containing an alkalizing pore-former agent, Chem. Eng. Des. (2011) 89, 1006-1010

4. Elnazeer I. Hamedelniel, J. Bajdik, T. Sovány, P. Kása Jr., K. Pintye-Hódi: Effects of the wetting liquid and ethylcellulose on the properties of atenolol-containing pellets. JDDST (2011) 21, 195-200

\section{ABSTRACTS}

1. Bajdik János; Elnazeer I. Hamedelniel; Klára Pintye-Hódi: Nedvesítő folyadék hatása az extrudálással és szferonizálással előállított pelletek tulajdonságaira, Congressus Pharmaceuticus Hungaricus XIV. Budapest, Kongresszusi Központ 2009. november 13-15.

2. Elnazeer I. Hamedelniel, Bajdik János, Klára Pintye-Hódi: Optimization of matrix pellets with factorial design, 7th World Meeting on Pharmaceutics, Biopharmaceutics and Pharmceutical Technology, Malta 8-11. March 2010.

3. Elnazeer I. Hamedelniel. Bajdik János, Klára Pintye-Hódi: Development of matrix pellets prepared by extrusion and spheronization of Atenolol and alkalizing component to enhance bioavailability. PharmSciFair, Prague, 13-17, June, 2011.

4. Elnazeer I. Hamedelniel, Péter Kása Jr., Klára Pintye-Hódi, István Zupkó: Study the bioavailability of Atenolol with alkalizing component in rats, 4th BBBB-Bled International Conference on Pharmaceutical Science, Bled, 29 September-1 October, 2011. accepted 


\section{Contents}

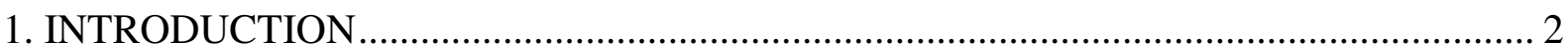

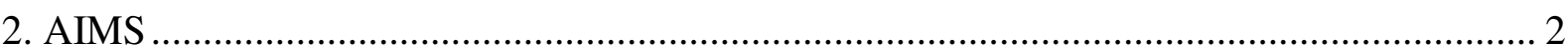

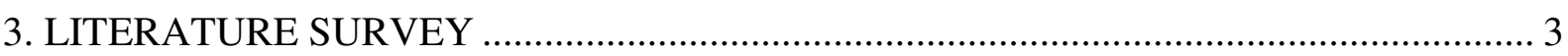

3.1. Pellets as a multiparticulate dosage form .............................................................. 3

3.2. Extrusion/spheronization pelletization process ...................................................... 4

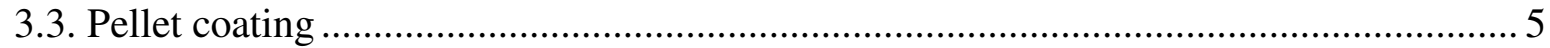

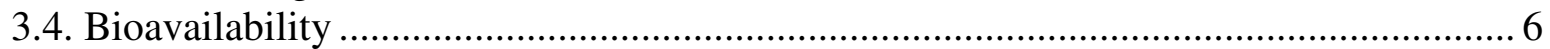

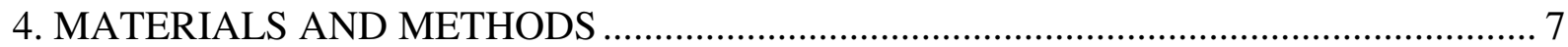

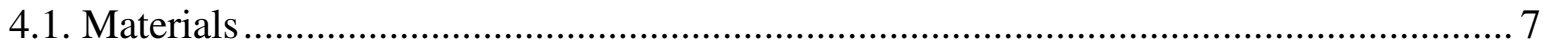

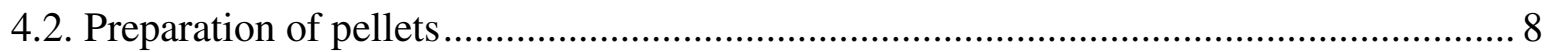

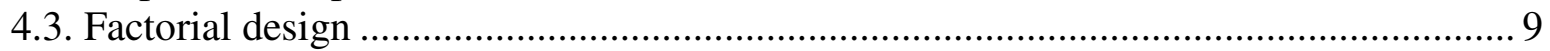

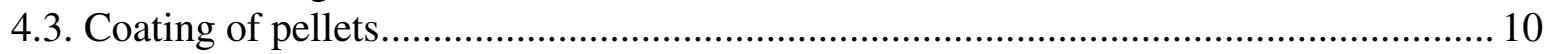

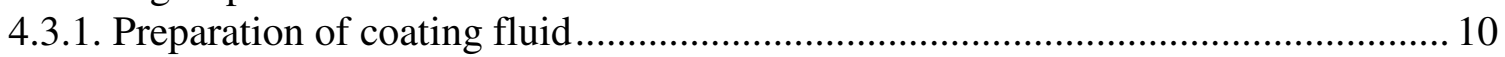

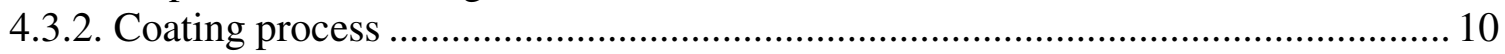

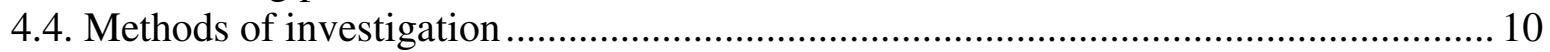

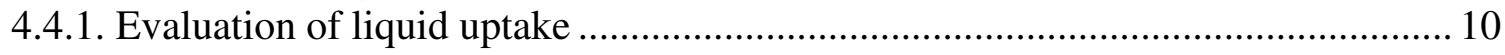

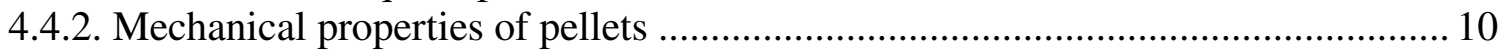

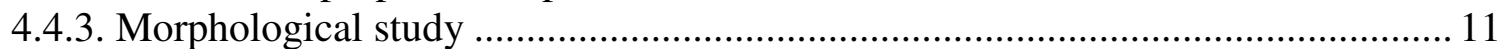

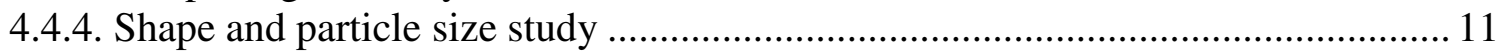

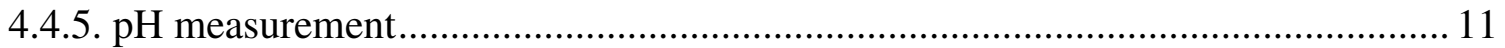

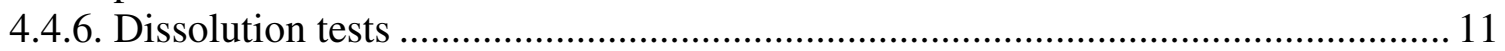

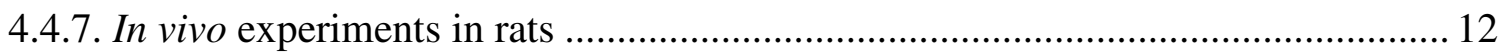

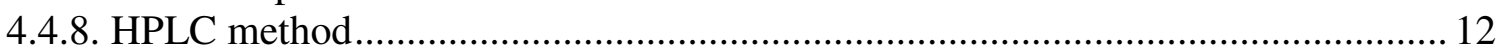

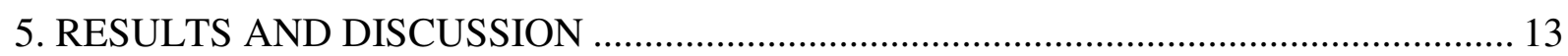

5.1. Determination of appropriate type of ethylcellulose as matrix-former ........................ 13

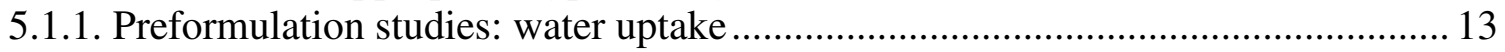

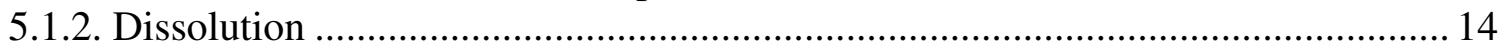

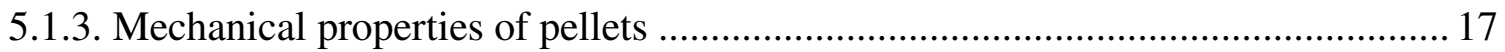

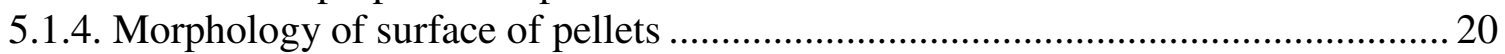

5.2. Determination of optimum wetting liquid and operational parameters ........................ 22

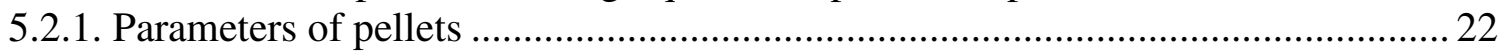

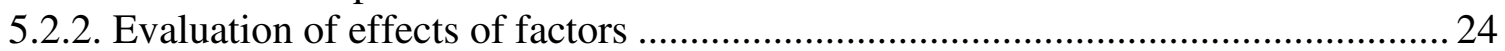

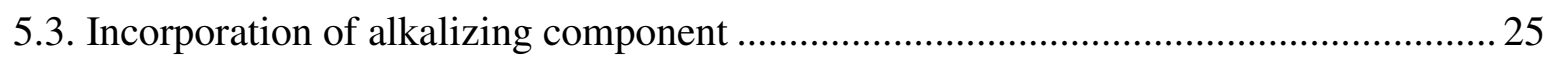

5.4. Preparation of pellets with optimum parameters and their coating .............................. 34

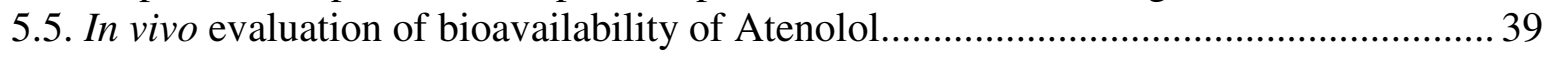

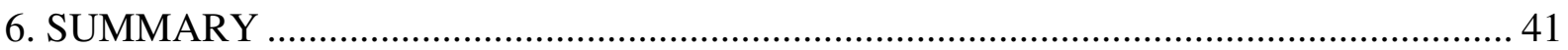

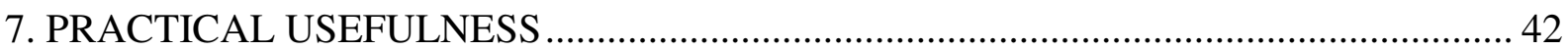

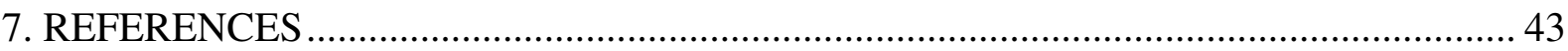




\section{INTRODUCTION}

Multiparticulate drug delivery systems are mainly oral dosage forms consisting of a multiplicity of small discrete units, each exhibiting some desired characteristics. Thus, multiparticulate dosage forms are pharmaceutical formulations in which the active pharmaceutical ingredient (API) is present as a number of small independent subunits. To deliver the recommended total dose, these subunits are filled into a sachet and encapsulated or compressed into a tablet [1].

The individual pellets in a multiparticulate system can sometimes be divided into fractions according to their size, coating, release properties, drug content, etc., offering a wide range of possibilities for drug development. Multiparticulate drug delivery systems are increasingly gaining favour on the market, as their multiple-unit nature furnishes several benefits over the more traditional single-unit dosage forms [2,3]. These include a lower irritative effect due to the decreased local concentration, less individual differences in plasma concentration than with tablets, a reduced risk of dumping, improved bioavailability, a large scale of products to be covered (in terms of both dosage forms and release kinetics) and an easy-to-solve approach to interactions [4-6].

\section{AIMS}

The primary aim of this study was the formulation of matrix pellets containing Atenolol (Atn) by means of extrusion/spheronization with a view to increasing its bioavailability. Pellets which undergo linear, but not too slow dissolution $(80 \%$ in the first hour) and with appropriate mechanical properties (because of the subsequent enteric soluble coating) are necessary for this purpose. Ethanol and water were used in various combinations as wetting liquid, and their effects on the properties of the pellets formed (breaking hardness and dissolution), on the formation of the matrix and on the interactions of the components were investigated. The Matrix-former polymer was ethylcellulose (EC).

I set out to investigate the effects of the parameters of spheronization on the properties of pellets containing Atn, microcrystalline cellulose (MCC) and EC without alkalizing components, and to determine the main factors which can influence the preparation of pellets.

During the processes, it is very important to ensure the best rheological properties for the wet mass. Water is generally used as wetting liquid. Since EC dissolves in ethanol, it was interesting to study the effects of ethanol or an ethanol-water mixture on the rheological properties of the wet mass and the final product. For this reason, one goal of the present study 
was to investigate the influence of an alkalizing component as pore-former and of the wetting liquid on the formulation of the pellets, and on their dissolution.

The fundamental aim of the present work was to study the delayed effects of matrix pellets coated with a gastric-resistant polymer on the release of Atn from pellets containing an alkalizing pore-former agent to ensure an appropriately alkaline micromilieu, and to improve the absorption of Atn from the intestine and therefore its bioavailability.

\section{LITERATURE SURVEY}

\subsection{Pellets as a multiparticulate dosage form}

Pellets for pharmaceutical applications are defined as spherical, free-flowing granules with a narrow size distribution, typically varying in diameter between 500 and $1500 \mu \mathrm{m}$. Pellets are a popular multiparticulate pharmaceutical dosage form that are utilized for both immediate release and a number of different controlled or special release applications [7-9]. In recent years, great efforts have been made to develop controlled drug release systems via which to achieve the optimum therapeutic effect of drugs; the drug concentration is maintained in the therapeutic window for a period of time, thereby ensuring sustained therapeutic action. In several diseases (such as bronchial asthma, hypertension, rheumatic disease and myocardial infarction) and for the control of body functions (blood pressure, and the levels of many hormones, e.g. aldosterone, renin and cortisol) influenced by circadian rhythms, delayed or pulsatile drug release could be the optimum approach [10-13]. Pellets offer biopharmaceutical advantages in terms of more even and predictable distribution and transportation in the gastrointestinal tract, which is fairly independent of the nutritional state [4]. The interest in pellets as a dosage form (filled into hard capsules) has increased continuously, for their multiparticulate nature offers important pharmacological and technological advantages over conventional single-unit solid dosage forms [14].

Particle morphology is a key determinant of the behaviour of bulk solids and multiparticulate systems: many of the physical and chemical properties of such systems depend on the particle shape and surface geometry [15]. Thus, the morphological characterization of particles is of great importance in pharmaceutical technology. In the field of granulation and pelletization, this characterization is critical for production steps such as capsule filling, and especially the coating of pellets. The morphological characterization of a particle basically requires three different aspects to be taken into account. First, the particle projection must be assigned to that geometric shape category (e.g. a circle) which best describes it. Second, the 
morphological analysis should include an assessment of the roundness of the particle, and the sharpness/roundness of the vertices, edges and sides of the particle. Third, the surface texture must be evaluated [16].

\subsection{Extrusion/spheronization pelletization process}

Pellets can be produced in different ways: spraying a solution or a suspension of a binder and API onto an inert core, a layering technique [17,18], spraying a melt of fats and waxes from the top into a cold tower (spray-congealing), forming pellets due to the hardening of the molten droplets [5], or spray-drying a solution or a suspension of the API forming pellets due to the evaporation of the fluid phase [5] spraying a binder solution into the whirling powder using a fluidized bed $[19,20]$. The popular method of producing pellets is by the extrusion/spheronization technique [5, 17-20].

The pelletization process consists of the agglomeration of fine powders of the APIs and excipients into small spherical units. The extrusion/spheronization pelletization process comprises five unit operations: blending, wet massing, extrusion, spheronization and drying. The extrusion of a wet powder mass leads to an intermediate spaghetti-like product, which is promptly spheronized to yield a final spherical product $[5,21]$. Since these phases are strongly related to each other [22], the quality of the end-product (pellets) is also strongly dependent on the process factors [23].

The physical characteristics of the pellets, which are directly affected by the process and formulation variables [24-27], highly influence the further manufacturing processes, such as film coating, capsule filling or tableting and consequently the properties of the final dosage form and its biological performance. It is very important to bear in mind that not all moistened powder mixtures can be successfully extruded and spheronized [28-30]. Newton defined the specific requirements for a wetted mass to be suitable for extrusion and spheronization. For a successful process, MCC is incorporated in most formulations, since it provides the wetted mass with the appropriate rheological properties [31, 32]. MCC may be regarded as the standard as a structure-forming material; it has good binding properties that provide cohesiveness to a wetted mass, in this way aiding extrusion/spheronization [33-37].

Moreover, in consequence of its large surface area and high internal porosity, it is able to absorb and retain a large quantity of water thereby facilitating extrusion, improving the wetted mass plasticity and enhancing spheronization [38]. Moreover, control of the movement of water through the plastic mass prevents phase separation during extrusion or spheronization [39]. 
Various pharmaceutical excipients can be used to modify the release of an API from pellets formulated by extrusion and spheronization. These components form a matrix system, which ensures appropriate liberation. Different types of polymers can be used to form soluble or insoluble systems. Their properties and the interactions between the components influence the dissolution of the API. EC is a highly suitable polymer for film coating [40-42] and a variety of types of Ethylcellulose are nowadays available as matrix-formers [43, 44]. EC has been widely used in oral pharmaceutical formulations for various purposes, including moisture protection, taste masking and controlled release. It is non-toxic, non-allergenic and non-irritant and has good film-forming properties [45, 46].

\subsection{Pellet coating}

Pellets are frequently coated in order to achieve sustained API release or to deliver an API to the specific absorption site in the gastrointestinal tract (e.g. enteric-coated or colon targeted API delivery) [5]. Enteric-coated pellets as dosage forms are especially suited for the administration of APIs which are not stable in the gastric fluids or which can cause irritation of the gastric mucosa and which are absorbed in the duodenum or upper intestine [47]. Several commercially available polymers are suitable for the coating of pharmaceutical dosage forms [48], and some can be used to control the API release kinetics. However, it is often difficult to adjust a particular release profile to the pharmacokinetic characteristics of the API. Different formulation and processing parameters can be varied in order to optimize the drug release patterns, e.g. coating level, type polymers, etc., but these variations are often restricted because reasonable film properties must be provided and production on a large scale must be feasible. To overcome these restrictions, polymer blends can be used as coating materials controlling API release [49-51].

The composition of the film coating and the nature of the coating technique (the use of aqueous dispersions or organic solutions) can affect the properties of the resulting polymeric membranes, including their permeability for water and API, mechanical resistance and dissolution behaviour [52-54]. Thus, for this purpose, various polymers are available, such as the generally accepted enteric polymer Eudragit L100-55 ${ }^{\circledR}$, which is soluble from pH 6.0 due to hydration of the ionized carboxylate groups [55]. There are organic solvent-based systems and aqueous dispersions.

The aqueous coating systems have numerous advantages over the organic solvent-based systems, for example with respect to ecological, toxicological and manufacturing safety concerns. However, the major limitation of many aqueous enteric coating formulations is the 
risk of premature API release (permeation) through the enteric coat in the stomach. This can be due to an increased permeability of aqueous film coatings [56, 57] or to a high watersolubility of the API [58]. Subcoating materials have been widely used in combination with enteric polymers to promote adhesion of the functional polymer [59], to function as a moisture barrier [60], and to prevent interactions between an API and an enteric coating [61]. Other researchers have described an increased gastric resistance of enteric-coated dosage forms in the presence of a polymeric sub-coat $[60,62,63]$. Our formula contained a high amount of a strong alkalizing agent, which influenced the dissolution of the Atenolol earlier than required. Hence, it was necessary to separate the core from the functional coating layer.

\subsection{Bioavailability}

Pharmacologically, bioavailability is a subcategory of absorption and is used to describe the fraction of an administered unchanged API that reaches the blood stream. When a medication is administered intravenously its bioavailability is $100 \%$ [64]. However, when a medication is administered via other routes eg, orally, its bioavailability generally decreases (due to incomplete absorption) and it may vary from patient to patient. Bioavailability is one of the essential tools in pharmacokinetics, as bioavailability must be considered when dosages are calculated for non-intravenous routes of administration.

For herbs and other nutrients in which the route of administration is nearly always oral, bioavailability generally designates simply the quantity or fraction of the ingested dose that is absorbed [65]. The pharmacological definition cannot apply to these substances because utilization and absorption is a function of the nutritional status and physiological state of the subject, resulting in even greater differences from individual to individual (interindividual variation) [66]. Therefore, bioavailability for dietary supplements can be defined as the proportion of the substance capable of being absorbed and available for use or storage [67].

The absolute bioavailability of an API, when administered by an extravascular route, is usually less than 1 (i.e. $<100 \%$ ). Various physiological factors reduce the availability of APIs prior to their entry into the systemic circulation. Whether an API is taken with or without food will also affect its absorption, other APIs taken concurrently may alter the absorption and first-pass metabolism, intestinal motility alters the dissolution of the API and may affect the degree of its chemical degradation by the intestinal microflora. Disease states affecting the liver metabolism or gastrointestinal function will also have an effect [68]. Other factors related to the API itself include: its physical properties, the formulation, whether the 
formulation is administered in a fed or a fasting state, the gastric emptying, circadian differences, and interaction with other APIs.

\section{MATERIALS AND METHODS}

\subsection{Materials}

The model API investigated was Atn (Ariane Organochem Private Ltd, Mumbai,India). Chemically, Atn is a phenylacetamide [(4-2-hydroxy-3-isopropylaminopropoxy) phenylacetamide]. It is a relatively polar, hydrophilic compound.

Atn is a cardioselective $\beta$-adrenoreceptor blocking agent used for the treatment of hypertension, including hypertension in pregnancy $[69,70]$. The absorption of the drug following oral administration in humans and most laboratory animal species is rapid, but incomplete (50-60\%). When given intravenously, Atn is almost completely excreted in the urine. Upon oral administration, between $40 \%$ and $50 \%$ of the unchanged compound is recovered in the urine and $50 \%$ is recovered unchanged in the faeces, due to incomplete intestinal absorption in the human and in most experimental species, except for the dog. There is no evidence that there is an alternative biliary route for excretion in either humans or experimental animals [71, 72]. Atn is well absorbed at $\mathrm{pH}>7.5$ [73]. The normal $\mathrm{pH}$ of the human ileum is 7.2-7.6, and thus total absorption is impossible. Co-administration of this Atn with an alkalizing component in a multiparticulate system is therefore reasonable in order to ensure an appropriately alkaline micromilieu. The good water solubility of Atn means that a polymer is required to control the parallel release of Atn and the alkalizing component.

Atn is incompletely absorbed from the human gastrointestinal tract with an absorption ranging from $40 \%$ to $50 \%$, and a bioavailability of just $36 \%$ of the whole dose [74]. The high polarity dictates its fast renal elimination with no significant metabolism. Moreover, the hypotensive effect of cardiovascular APIs will be brought into full play only if the blood pressure is reduced steadily [75]. Therefore, many efforts have been made to improve pharmaceutical formulation in order to optimize the therapy. These efforts have been focused on the development of oral sustained-release preparations, which could increase the time available for API absorption, leading to an increase in its bioavailability, and a decrease in fluctuation of its plasma concentration, which reduces the side-effects. Accordingly, studies have been reported on the regulation of Atn release through the use of diverse controlled release systems such as osmotic pumps [76-79], mucoadhesive microspheres [80], 
mucoadhesive tablets [81], transdermal delivery systems [82, 83] and floating controlled delivery systems $[84,85]$.

In adult non-pregnant subjects, the disposition of Atn has been studied in humans and in several animal species: rats, mice, rabbits, dogs, and rhesus monkeys [86, 87]. The absorption of Atn upon oral administration in humans and most laboratory animal species is rapid but incomplete. Due to its incomplete intestinal absorption, the systemic bioavailability is about $50 \%$ to $60 \%$ in the human [88], rat, mouse, rabbit, and monkey [71]. In contrast, in the dog, absorption from the gut is almost complete: $98 \%$ of the total dose [86].

EC (Ethocel standard 4, 10 and 45 premium, Colorcon Ltd. Dartford, England) was used as pharmaceutical matrix-former.

MCC (Vivapur 103, Rettenmaier\&Söhne GmbH, Rosenberg, Germany) was used as pharmaceutical excipients structure-former.

Ethanol 96\% (Spectrum 3D, Debrecen, Hungary) and water were applied as wetting components.

Trisodium phosphate dodecahydrate $\left(\mathrm{Na}_{3} \mathrm{PO}_{4} * 12 \mathrm{H}_{2} \mathrm{O}\right)$ (VWR International, Belgium) and disodium phosphate anhydrous $\left(\mathrm{Na}_{2} \mathrm{HPO}_{4}\right)$ (Spektrum 3D, Debrecen, Hungary) were used as alkalizing and pore-former agents.

Opadry clear (hydroxylpropyl methylcellulose (HPMC) and Acryl EZE MP (Colorcon Ltd., Dartford, England) were utilized as coating dispersions. The latter contains methacrylic acid copolymer (Eudragit L100-55) ${ }^{\circledR}$ as enteric coated material and plasticizer, which are necessary during coating. Dimethicone (Silfar E 1049, Wacker Chemie AG, supplier: Brenntag Hungaria Kereskedelmi Kft, Hungary) was used as anti-foaming agent.

Ariavit sunset yellow CI 15985 (Sensient Food Colors Hungary Kft, Hungary), Erythrosin 6560199 (Sicopharm BASF Germany) and Indigo carmine E132 were used as dyes.

\subsection{Preparation of pellets}

$150 \mathrm{~g}$ of powder mixture was prepared from Atn and excipients (EC, MCC and alkalizing component). To obtain a uniform mixture, the powder was blended at $50 \mathrm{rpm}$ for 10 min with a Turbula mixer (W.A. Bachofen, Basel, Switzerland).

Samples were prepared in a high-shear granulator (ProCepT 4M8 granulator, ProCepT nv, Zelzate, Belgium) with a sufficient amount of granulating liquid, i.e. either water alone, or a combination of water and ethanol $(80 \mathrm{ml} / 15 \mathrm{ml}$, respectively). The kneading parameters 
were as following. Impeller speed: $1500 \mathrm{rpm}$, Chopper speed: $2000 \mathrm{rpm}$ and Dosing speed: 5 $\mathrm{ml} / \mathrm{min}$.

The wet mass obtained was extruded by a mini screw extruder (Caleva Ltd. Sturminster Newton, Dorset, UK) equipped with an axial screen with dies $1 \mathrm{~mm}$ in diameter and $4 \mathrm{~mm}$ in length, operating at $90 \mathrm{rpm}$. The jacked barrel of the extruder was cooled by water at $25 \pm 2{ }^{\circ} \mathrm{C}$. Each extrudate was collected in a container before it was spheronized. About $40 \mathrm{~g}$ of extrudate was spheronized at a time, on a $12 \mathrm{~cm}$ diameter spheronizer (Model120, G.B. Caleva Ltd. Sturminster Newton, Dorset, UK) fitted with a cross-hatch grooved plate, for $3 \mathrm{~min}$ and $10 \mathrm{~min}$ at $750 \mathrm{rpm}$ and $1000 \mathrm{rpm}$. The pellets were dried under the same conditions at $40 \pm 2{ }^{\circ} \mathrm{C}$ for $24 \mathrm{~h}$.

\subsection{Factorial design}

The factorial design is a method often used to accelerate the solution of problems. This method has been utilized in various branches of science and industry, e.g. food research [86], environmental management [89], chemistry [90] and pharmaceutical technology [91-96]. The mathematically determined effects of different factors are compared by means of this technique, this information being very useful for the application of process analytical technology, at the heart of which is the acquisition of a deep understanding of the manufacturing process [97]. A $2^{3}$ full factorial design was applied to choose the relevant factors (granulation liquid, water alone or with ethanol, spheronization speed and duration of spheronization) influencing three operational parameters: the dissolution, the breaking strength of the pellets and the shape of the pellets (sphericity). The levels of the factors are to be found in section 5.2.1. The experiments were performed in randomized sequence. The samples are also designated in section 5.2.1. The following approach, containing the interactions of the factors, was used to determine the response surface and the relative effects of the factors (b):

$$
y=b_{0}+b_{1} x_{1}+b_{2} x_{2}+b_{3} x_{3}+b_{12} x_{1} x_{2}+b_{13} x_{1} x_{3}+b_{23} x_{2} x_{3}
$$

Statistica for Windows 8.1 AGA software (StatSoft, Inc. Tulsa, USA) was used for the calculations. 500 pellets of each sample were checked. During the mathematical evaluations, the confidence interval was $95 \%$, i.e. the differences were significant if $p<0.05$. 


\subsection{Coating of pellets}

\subsubsection{Preparation of coating fluid}

a) Opadry solution for subcoating

Our formula contained an alkalizing component which may interact with the functional coating film, a protecting layer was therefore necessary and was prepared as follows:

$600 \mathrm{ml}$ of distilled water was stirred at $400 \mathrm{rpm}$ for $5 \mathrm{~min}$, during which $5 \%$ of Opadry clear was added in portions, and the resulting mixture was shaken gently and stirred at $200 \mathrm{rpm}$ for $1 \mathrm{~h}$, and then filtered.

\section{b) Acryl EZE MP dispersion liquid for coating}

$120 \mathrm{~g}$ of Acryl EZE MP and $1 \mathrm{~g}$ of dimethicone were weighed and added in portions to $479 \mathrm{~g}$ of distilled water in a beaker during stirring at $400 \mathrm{rpm}$ for at least $2 \mathrm{~h}$, and the dispersed liquid was then passed through a $0.25 \mathrm{~mm}$ sieve to obtain a dispersion system with uniform particle size with a suitable dye as colouring agent.

\subsubsection{Coating process}

$200 \mathrm{~g}$ of pellet cores was coated in a fluidized bed coater equipped with a Wurster column (Strea 1; Aeromatic-Fielder, Bubendorf, Switzerland). The process parameters were as follows: inlet temperature $40 \pm 2{ }^{\circ} \mathrm{C}$, product temperature $50 \pm 2{ }^{\circ} \mathrm{C}$, spray rate $2.5-3 \mathrm{~g} / \mathrm{min}$, atomization pressure 2 bar, nozzle diameter $1 \mathrm{~mm}$, and air volume $95 \mathrm{~m}^{3} / \mathrm{h}$. After coating, the beads were further fluidized for $10 \mathrm{~min}$ and subsequently cured in an oven for $24 \mathrm{~h}$ at $40{ }^{\circ} \mathrm{C}$.

\subsection{Methods of investigation}

\subsubsection{Evaluation of liquid uptake}

The Enslin number is a simple semiquantitative measure of the liquid (here water and ethanol) uptake of a powder and is equal to the amount of fluid absorbed by $1 \mathrm{~g}$ of powder (ml/g). An Enslin apparatus with a glass filter and a pipette with $0.01 \mathrm{ml}$ accuracy were used for these experiments [98]. A monolayer of particles took up the maximum quantity of liquid possible through a filter paper under these conditions. $0.5 \mathrm{~g}$ of each powder was tested; three parallel experiments were performed.

\subsubsection{Mechanical properties of pellets}

The breaking strength of pellets was tested. The strength tester and the software were developed in our institute. The tester contains a special specimen holder and a jowl, and is 
connected to a computer via an interface. Thus, not only can the ultimate deformation force be measured, but the process (force-time and force-displacement curves) can be followed. The specimen is located horizontally on a special plate and the jowl moves vertically.

The measurement range was 0-200 N, the speed of the stamp was $20 \mathrm{~mm} / \mathrm{min}$, and the output was $0-5 \mathrm{~V}$. The sensor was a Unicell force- measuring instrument, calibrated with the C9B $200 \mathrm{~N}$ cell.

\subsubsection{Morphological study}

The surfaces of various samples before and after dissolution were tested with a scanning electron microscope (SEM) (Hitachi S4700, Hitachi Scientific Instruments Ltd., Tokyo, Japan). A sputter coating unit (Polaron E5100, VG Microtech, UK) was used to charge the surfaces for the SEM measurements. The air pressure during the analyses was 1.3$13 \mathrm{mPa}$.

\subsubsection{Shape and particle size study}

The particle size and the shape of the pellet surface were studied by using a system consisting of a stereomicroscope (Zeiss Stemi2000-C, Carl Zeiss GmbH, Vienna, Austria) and a ring light with a cold light source (Leica KL 1500, Cambridge, UK). A Quantimet 500 (Q500MC) image processing and analysis system (Leica Cambridge Ltd., Cambridge, UK) was used. The aspect ratio was utilized for the evaluation of the shape of the particles.

\subsection{5. $\mathrm{pH}$ measurement}

$0.1 \mathrm{~g}$ of pellets was weighed and placed in a beaker containing $50 \mathrm{ml}$ of distilled water or buffer ( $\mathrm{pH}=6.8$ ), which was agitated magnetically. A small amount of liquid was used for the testing to determine the effect on the micromilieu. After each $10 \mathrm{~min}$ of stirring $(120 \mathrm{rpm})$, the pellets were filtered off and the $\mathrm{pH}$ of the solution was measured. To measure the changes in $\mathrm{pH}$ with time, the filtered pellets were again placed in $50 \mathrm{~mL}$ of distilled water or buffer. This process was repeated until the $\mathrm{pH}$ remained unchanged. Each test was repeated three times.

\subsubsection{Dissolution tests}

Pellets $(100 \mathrm{mg})$ were filled into capsules, which were placed into the basket of a dissolution tester (Erweka DT 700, Heusenstamm, Germany). The dissolution medium consisted of $900 \mathrm{ml}$ of phosphate buffer $\left(\mathrm{pH}\right.$ 6.8) kept at $37.0 \pm 0.5{ }^{\circ} \mathrm{C}$. The rotational speed of the baskets was set at $100 \mathrm{rpm}$. The dissolution system was combined with an automatic sampling station. Samples of $5 \mathrm{ml}$ were withdrawn at 10, 20, 30, 40, 50 and $60 \mathrm{~min}$. Atn was 
measured spectrophotometrically (Unicam He $\lambda$ ios Alpha, Spectronic Unicam, Cambridge, $\mathrm{UK}$ ) at $\lambda_{\max }=224 \mathrm{~nm}$. For coated pellets filled into HPMC (hydroxyl propyl methylcellusose) capsules, dissolution studies $(n=3)$ were carried out in $900 \mathrm{ml}$ of $\mathrm{HCl} / \mathrm{NaCl}$ solution ( $\mathrm{pH}$ 1.2). After $2 \mathrm{~h}$, the $\mathrm{pH}$ of the medium was changed from 1.2, to 6.8 to simulate the gastric transition, according to the European Pharmacopoeia [27]. Samples of $5 \mathrm{ml}$ were withdrawn at 10, 30, 60, 90 and $120 \mathrm{~min}$ from the $\mathrm{HCl}$ solution at $\mathrm{pH} 1.2$ and at 10, 20, 30, 40, 50, 60, 90, 120 and $180 \mathrm{~min}$ from the phosphate buffer at $\mathrm{pH}$ 6.8. The content of Atn was measured spectrophotometrically (Unicam He $\lambda$ ios Alpha, Spectronic Unicam, Cambridge, UK) at $\lambda_{\max }=202 \mathrm{~nm}(\mathrm{HCl} / \mathrm{NaCl} \mathrm{pH} 1.2)$ and $224 \mathrm{~nm}$ (phosphate buffer $\mathrm{pH}$ 6.8).

\subsubsection{In vivo experiments in rats}

Male SPRD rats (190-210 g) were fasted for $16 \mathrm{~h}$ and pellet-filled capsules were then administered orally at $30 \mathrm{mg} / \mathrm{kg}$ Atn. After 2, 4, 6 and $8 \mathrm{~h}$, animals were sacrificed and serum samples were prepared for analysis by HPLC.

\subsubsection{HPLC method}

Stock solution of Atenolol was prepared in $\mathrm{MeOH}(1 \mathrm{mg} / \mathrm{ml})$. Solutions of 1, 10 and $100 \mu \mathrm{g} / \mathrm{ml}$ were prepared by diluting the stock solution in $\mathrm{MeOH}$ as standard solution. To 500 $\mu \mathrm{l}$ of standard solution or test serum sample, $200 \mu \mathrm{l}$ of $\mathrm{NaOH}(0.25 \mathrm{M})$ was added. After a brief vortex mixing, $3.5 \mathrm{ml}$ of the extraction solvent (hexane: $n$-butanol, 1:1) was added, and the compounds of interest were extracted by vortex mixing for $20 \mathrm{~s}$. The tubes were then centrifuged $\left(14000 \mathrm{rpm}\right.$ at $25{ }^{\circ} \mathrm{C}$ for $\left.10 \mathrm{~min}\right)$ and the organic layer was transferred to another set of clean tubes to be back-extracted with $250 \mu \mathrm{l}$ of $0.1 \mathrm{M}$ hydrochloric acid (vortex mixing for $20 \mathrm{~s})$. The tubes were then centrifuged $\left(14000 \mathrm{rpm}\right.$ at $25^{\circ} \mathrm{C}$ for $\left.10 \mathrm{~min}\right)$, the organic layer was discarded, and the aqueous phase was transferred to a clean tube to be evaporated to dryness at $40{ }^{\circ} \mathrm{C}$, under an $\mathrm{N}_{2}$ stream. The residue was dissolved with $100 \mu \mathrm{l}$ of the mobile phase and transferred to an injection vial, and a $20 \mu \mathrm{l}$ aliquot was injected into the chromatographic system. The mobile phase consisted of a mixture of ACNMeOH $0.01 \mathrm{M}$ phosphate buffer with the $\mathrm{pH}$ adjusted to 6.0 with $\mathrm{NaOH}$, containing $0.1 \%$ SDS (15:60:25 vol $/$ volvol), pumped at a flow rate of $1.2 \mathrm{ml} / \mathrm{min}$ through the column (Lichrosorb, $10 \mu \mathrm{m} \mathrm{RP}$ 18-250×4.6 mm (Merck) with a guard column Security-guard, Phenomenex, CA) at room temperature. Peaks were monitored by fluoresce $\left(\lambda_{\mathrm{EX}}=258 \mathrm{~nm}, \lambda_{\mathrm{EM}}=300 \mathrm{~nm}\right)$, in high sensitivity mode at $23{ }^{\circ} \mathrm{C}$. Atn was Quantitated by plotting Atn to internal standard peak height ratios as a function of the concentrations [98]. 


\section{RESULTS AND DISCUSSION}

\subsection{Determination of appropriate type of ethylcellulose as matrix-former}

\subsubsection{Preformulation studies: water uptake}

The uptake of liquids applied during pelletization is a very important parameter for the preparation of beads. The different components were tested. The sample compositions and granulation liquids are shown in the Table 1, and the liquid uptakes in Table 2.

Table 1. Sample compositions

\begin{tabular}{|c|c|c|c|c|c|c|c|c|c|}
\hline \multicolumn{2}{|c|}{ Sample } & \multicolumn{3}{|c|}{ Powder } & \multicolumn{4}{|c|}{ Binder liquid } & \multirow{3}{*}{$\begin{array}{c}\text { Total } \\
\text { liquid } \\
\text { amount } \\
(\mathrm{ml})\end{array}$} \\
\hline & & \multirow{2}{*}{$\begin{array}{c}\text { At } \\
(\%)\end{array}$} & \multirow{2}{*}{$\begin{array}{c}\text { MCC } \\
(\%)\end{array}$} & \multirow{2}{*}{$\begin{array}{l}\mathrm{EC} \\
(\%)\end{array}$} & \multicolumn{2}{|c|}{ Water/ml } & \multicolumn{2}{|c|}{ Ethanol/ml } & \\
\hline & & & & & Pre & Post & Pre & Post & \\
\hline \multirow[t]{3}{*}{$\mathrm{S} 1$} & A & 60 & 20 & 20 (EC4) & 95 & & - & - & 95 \\
\hline & B & 60 & 20 & 20 (EC10) & 95 & & & & 95 \\
\hline & $\mathrm{C}$ & 60 & 20 & 20 (EC45) & 95 & & & & 95 \\
\hline \multirow[t]{3}{*}{ S2 } & $\mathrm{A}$ & 60 & 20 & 20 (EC4) & & 80 & 15 & & 95 \\
\hline & B & 60 & 20 & 20 (EC10) & & 80 & 15 & & 95 \\
\hline & $\mathrm{C}$ & 60 & 20 & $20(\mathrm{EC} 45)$ & & 80 & 15 & & 95 \\
\hline \multirow[t]{3}{*}{ S3 } & A & 60 & 20 & 20 (EC4) & 80 & & & 15 & 95 \\
\hline & B & 60 & 20 & 20 (EC10) & 80 & & & 15 & 95 \\
\hline & $\mathrm{C}$ & 60 & 20 & 20 (EC45) & 80 & & & 15 & 95 \\
\hline \multirow[t]{3}{*}{ S4 } & $\mathrm{A}$ & 60 & 20 & 20 (EC4) & \multirow{3}{*}{\multicolumn{4}{|c|}{$\begin{array}{l}\text { Mixture of } 80 \mathrm{ml} \text { water } \\
+15 \mathrm{ml} \text { ethanol }\end{array}$}} & 95 \\
\hline & $\mathrm{B}$ & 60 & 20 & $20(\mathrm{EC} 10)$ & & & & & 95 \\
\hline & $\mathrm{C}$ & 60 & 20 & 20 (EC45) & & & & & 95 \\
\hline
\end{tabular}

Table 2. Liquid uptakes of different samples, as Enslin numbers ( $\mathrm{ml} / \mathrm{g})$

\begin{tabular}{|l|c|c|c|c|c|}
\hline \multirow{2}{*}{ Liquid } & \multicolumn{5}{|c|}{ Type of powder } \\
\cline { 2 - 6 } & EC4 & EC10 & EC45 & MCC & Atn \\
\hline Water & $0.05 \pm 0.01$ & $0.07 \pm 0.02$ & $0.15 \pm 0.01$ & $2.76 \pm 0.04$ & $0.07 \pm 0.02$ \\
\hline Ethanol & $0.25 \pm 0.02$ & $0.29 \pm 0.02$ & $0.61 \pm 0.01$ & $1.88 \pm 0.14$ & $1.09 \pm 0.05$ \\
\hline Water+ethanol* & $0.11 \pm 0.01$ & $0.23 \pm 0.01$ & $0.41 \pm 0.01$ & $2.71 \pm 0.02$ & $0.64 \pm 0.14$ \\
\hline
\end{tabular}

$* 80 \mathrm{ml}$ water $+15 \mathrm{ml}$ ethanol

The highest liquid uptake for both water and ethanol was detected for MCC and the lowest for EC4 (Table 2). MCC has been described as a 'molecular sponge' [16]. The MCC particles are able to absorb and retain water in a manner similar to a sponge. The uptake of the mixture of water and ethanol applied was very similar to the water uptake for MCC. The uptake of ethanol by Atn was higher than that of water. As concerns the different grades of $\mathrm{EC}$, it is clear that, independently of the composition of the liquid, EC45 took up the highest 
amount of liquid. A gel-like layer formed around the particles in the ethanol. The viscosity of this mucous layer is known to depend on the type of EC (Colorcon brochure).

\subsubsection{Dissolution}

On the basis of the liquids uptake results, pellets were prepared and tested (Table 1). The drug release from the pellets was studied with different dissolution kinetic models (firstorder, Higuchi, Hopfenberg, RRSBW and Langenbucher). The results showed that the dissolution profile of the samples could be fitted best $\left(R^{2}>0.95\right)$ with first-order kinetics (Eq. 1 ), as is expected for the dissolution of water-soluble drugs from porous matrices.

$$
M_{t}=M_{0}\left(1-e^{-k t}\right)
$$

where $M_{t}$ is the amount of API released from the preparation in time $t, M_{0}$ is the total amount of the drug, and $k$ is the dissolution rate of the process. However, even this model was unable to handle the presence of a lag time, which can be observed especially in the case of samples prepared with ethanol. The dissolution was fitted with the Chapman-Richards growth model (Eq. 2), which contains a shape parameter of the sigmoid-shaped curve (Figs 1-3), and can describe the lag time:

$$
M_{t}=M_{0}\left(1-e^{-k t}\right)^{c}
$$

where $M_{t}$ is the amount of API released from the preparation in time $t, M_{0}$ is the total amount of the drug, $k$ is the dissolution rate of the process, and $c$ is the shape parameter of the curve, which refers to the observed lag time of the dissolution. The dissolution rates and shape parameters are displayed in Table 3.

The results were analysed with a two-way ANOVA model. The results showed significant differences in the rates of dissolution of the samples. On the basis of these statistical differences, the samples can be divided into three groups. The highest dissolution rates were observed for samples S2B and S3C, those for samples S3B and S4C were onetenth less, and the other samples displayed even lower dissolution rate constants (Table 3). 


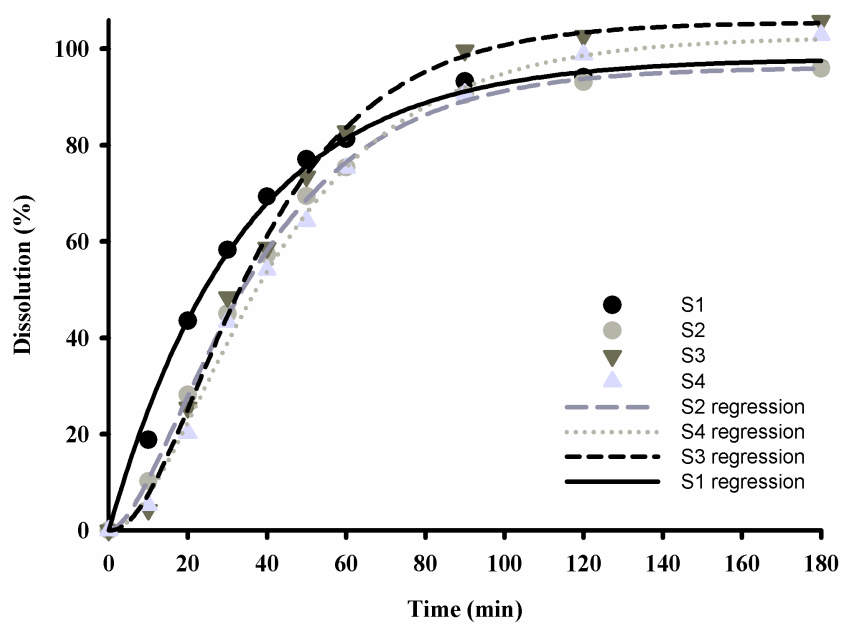

Fig. 1. Dissolution of Atn from pellets containing EC4

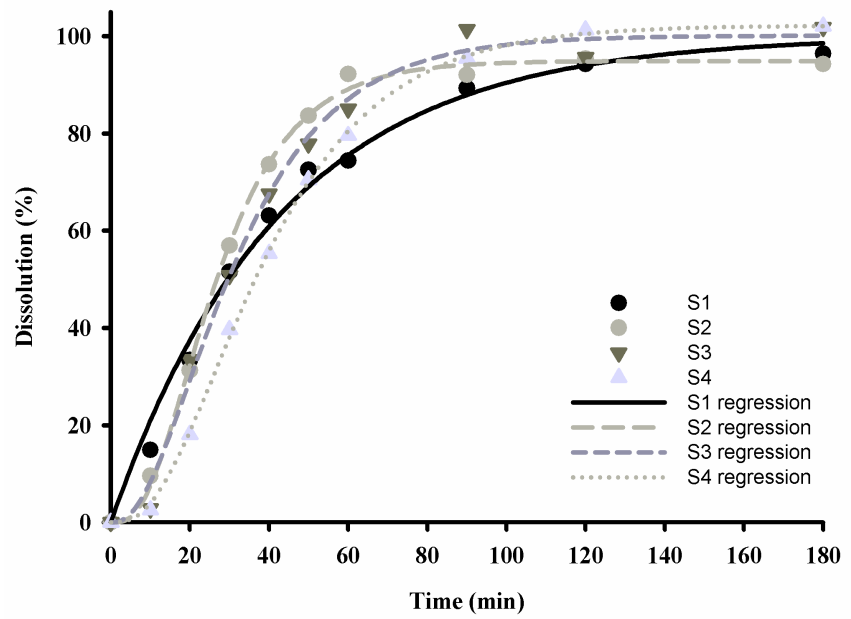

Fig. 2. Dissolution of Atn from pellets containing EC10

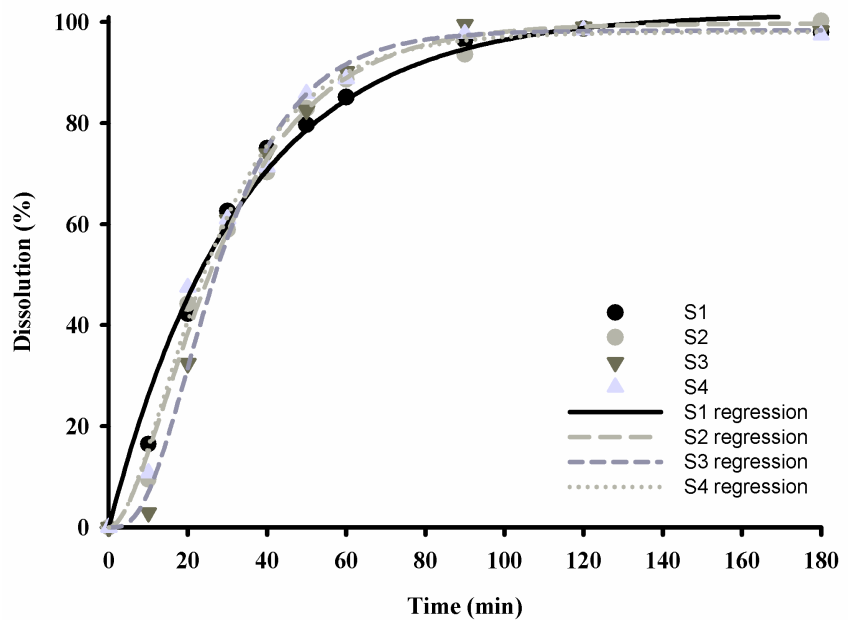

Fig. 3. Dissolution of Atn from pellets containing EC45 

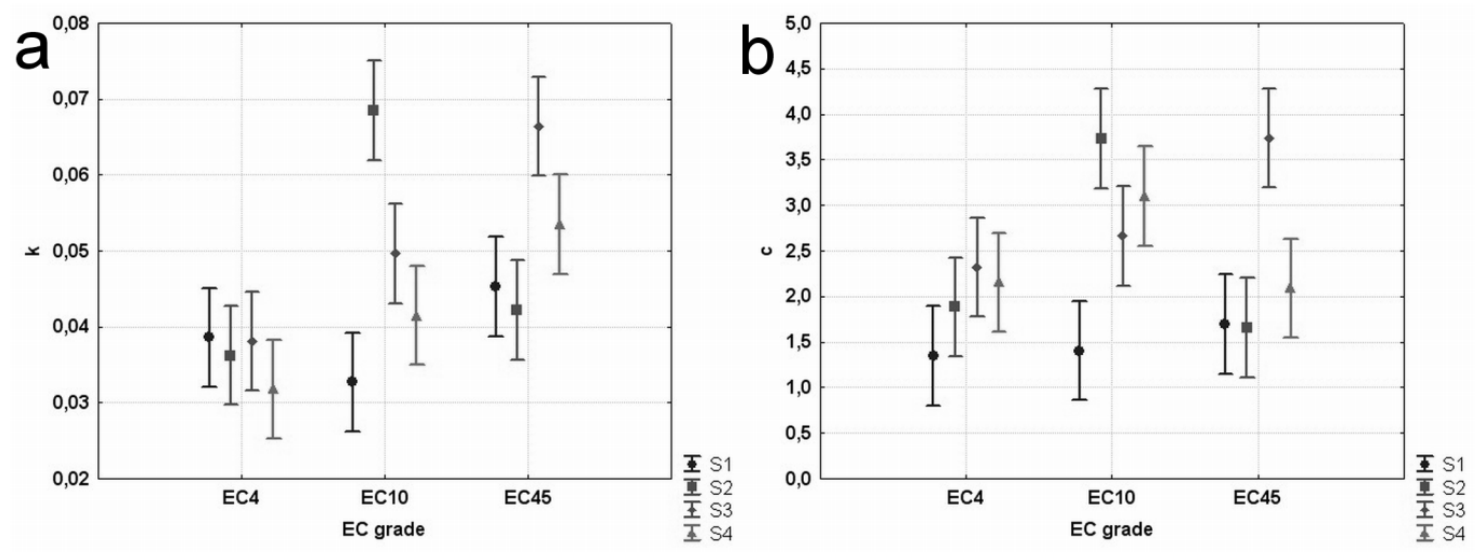

Fig. 4. Box-plots of the statistical analysis: effects of the EC grade and the method of preparation for the dissolution rate constants (a) and lag-time (b)

A similar tendency was seen in the shape of the dissolution curves. There was a considerable lag time for samples with higher dissolution rates. The lag time was significantly shorter (Fig. 4b) for samples prepared with water than for samples prepared with water and ethanol, with the exception of sample S2C. A possible explanation of the manifestation of the lag time, therefore, is that the ethanol dissolves EC, and during the drying period an EC film is formed around the drug particles, this layer delaying the dissolution. Nevertheless, the mechanism and degree of film formation differed for the different grades of EC. These differences are well revealed by the shifts in the maximum dissolution rates (Fig. 4a) and lag times (Fig. 4b). A possible explanation of this phenomenon is that the quality of the films is sensitive to the solvent mixture applied, depending on the chain-length of the EC. In the case of short chain-length ECs (EC4), the films formed are rigid and break easily, and are not able to influence the drug dissolution. The quality of the films improves significantly with the lengthening of the polymer chains, and the film formation can then modify the rate of dissolution of the drug. On the basis of these results, the samples prepared with water were chosen for further investigation. 
Table 3. Dissolution rate constants and correlation coefficients of dissolution curves

\begin{tabular}{|l|l|c|c|c|}
\hline \multicolumn{2}{|l|}{ Sample } & $R^{2}$ values & $\begin{array}{c}\text { Dissolution rate } \\
\text { constants }\end{array}$ & Shape parameter \\
\hline S1 & A & 0.9968 & 0.04 & 1.35 \\
\hline & B & 0.9919 & 0.03 & 1.40 \\
\hline & C & 0.9956 & 0.05 & 1.70 \\
\hline S2 & A & 0.9951 & 0.04 & 1.88 \\
\hline & B & 0.9928 & 0.07 & 3.73 \\
\hline & C & 0.9870 & 0.04 & 1.66 \\
\hline S3 & A & 0.9871 & 0.04 & 2.32 \\
\hline & B & 0.9875 & 0.05 & 2.66 \\
\hline & C & 0.9929 & 0.07 & 3.74 \\
\hline S4 & A & 0.9903 & 0.03 & 2.16 \\
\hline & B & 0.9968 & 0.04 & 3.10 \\
\hline & C & 0.9895 & 0.05 & 2.09 \\
\hline
\end{tabular}

\subsubsection{Mechanical properties of pellets}

The evaluation of the process of pellet deformation involved not only determination of the breaking hardness, but also study of the deformation curve. The shapes of the breaking curves of the pellets were very similar for the samples prepared with water (Fig. 5). They mainly comprised three phases: The first section (Fig. 5A:1) is indicative of elastic deformation. The pellet behaves as a Kelvin body, in which the Hooke component dominates. The relationship between the loading and the stress can be written as follows:

$$
t=E(d-\delta)
$$

where $t=$ the Cauchy stress tensor, $E=$ the elasticity modulus, $d=$ the tensor of deformation and $\delta=$ unit tensor.

In the second section (Fig. 5A:(2), the pellet behaves as a Saint-Venant body, exhibiting a permanent deformation after reaching the breaking stress (plastoelastic section), which leads to breaking of the crystal structure: third section (Fig. 5A: 3). During the total deformation process, the behaviour of the binder film as a Hooke body predominates until the total breaking of the pellet. The samples containing ethanol gave curves with more peaks. This phenomenon revealed that the internal structure of these samples was inhomogeneous.

A probable liquid-independent factor determining the pellet structure could be the plastic deformation of the particles containing EC during the extrusion. However, other liquid-dependent structure-determining components cause changes in the shape of the deformation curve. 

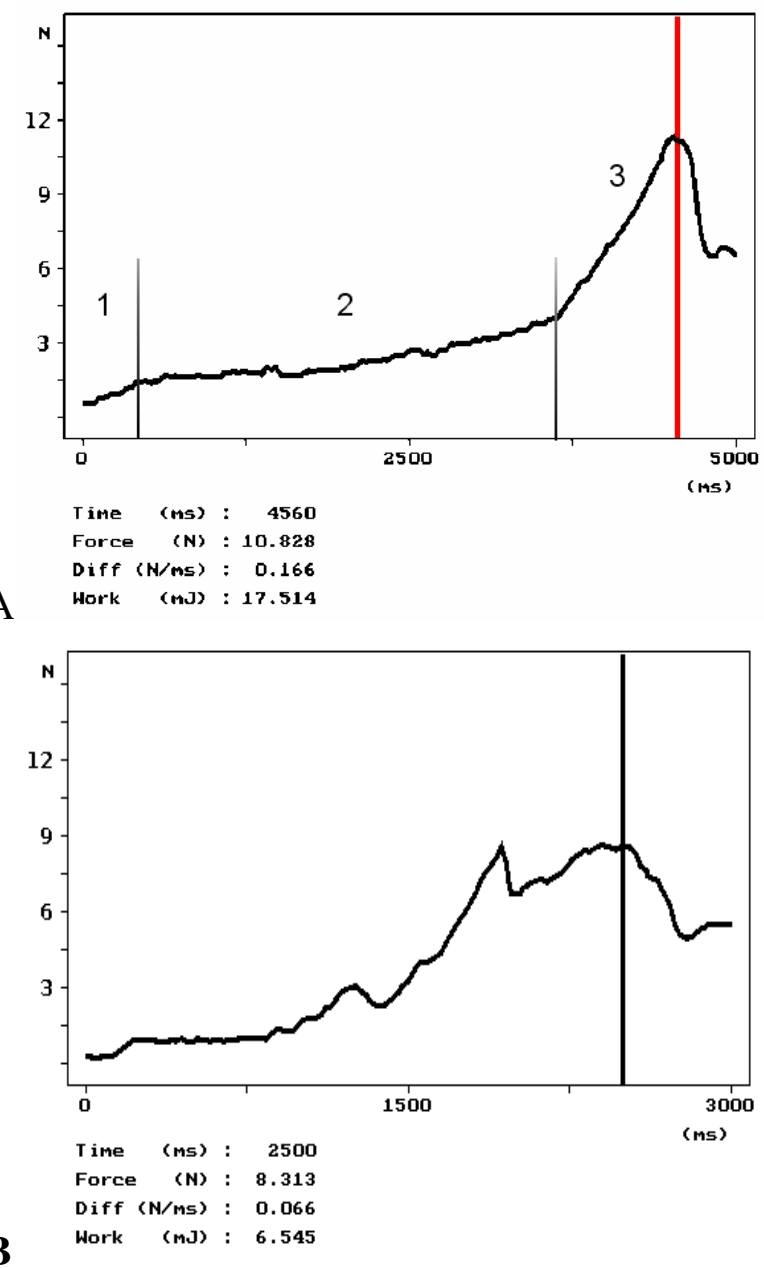

Fig. 5. Deformation curves of pellets containing EC45 (A: S1C., B: S3C)

For the samples prepared in water, the main binder in the pellet was the MCC. It is known that, according to the 'crystallite-gel model' [31], MCC particles are broken down into smaller units and even partly into single crystals of colloidal size during granulation and extrusion in the presence of water. The resulting crystallites and porous particles form a coherent gel-like network (with a high fraction of an insoluble solid phase) and immobilize the liquid. Over a particular range of water content, which relates to acceptable gel strength, extrusion and spheronization become possible. This effect improves the plasticity of the wetted mass and enhances the spheronization, and is therefore a pellet structure-forming parameter which can determine the mechanical properties. In this case, other possible structure-forming phenomena could include the recrystallization of Atn and the partial melting of EC during the extrusion. The presence of ethanol in the wetting process relevantly influenced the gel formation and solubility of Atn. The film-forming mucous solution of EC could change the structure of the "MCC gel". The viscosity of this ethanolic solution exerted a disturbing effect. The inhomogeneity in the "MCC gel-like" matrix and the film formation of 
EC had relevant effects on the properties of the pellets. A lower breaking hardness of samples prepared with ethanolic solution could be detected in the case of ECs with higher viscosity grades (Table 4). The difference appeared to be significant in the two-way ANOVA test. There was no significant effect of the wetting liquid on the mechanical properties of the pellets prepared with EC4. This can be explained by the low viscosity of its ethanolic solution. Its effect on the plasticity of the mass was therefore negligible. In the case of watercontaining samples, there was not a significant decreasing tendency with increasing water uptake of the EC. The effect of the hydrated EC therefore increased, and accordingly the breaking hardness for S1C decreased. For each liquid, the lowest breaking force was detected for the sample containing EC45. The samples prepared with this polymer exhibited decreases in breaking force in the sequence water $>$ aqueous ethanol solution. The extent of the alteration was similar in every case.

A reduction in breaking hardness was also detected for EC10, but it exhibited a less considerable change for S4B. In this case, the aqueous ethanol did not give rise to a more dramatic change in the "crystallite-gel model" of MCC. The liquid uptake of MCC was very similar for water and aqueous ethanol, and the presence of an EC gel can therefore disturb the formation of the particles. The hydration of EC10 induced a lower extent of alteration than that for EC45, the hydration of which is better in aqueous ethanol.

Table 4. Breaking hardness of pellets

\begin{tabular}{|l|l|c|}
\hline \multicolumn{2}{|l|}{ Sample } & $\begin{array}{c}\text { Breaking force } \\
(\mathrm{N})\end{array}$ \\
\hline \multirow{3}{*}{ S1 } & A & $13.7 \pm 2.5$ \\
\cline { 2 - 3 } & B & $11.9 \pm 2.2$ \\
\cline { 2 - 3 } & C & $11.2 \pm 1.3$ \\
\hline S2 & A & $13.5 \pm 1.6$ \\
\cline { 2 - 3 } & B & $8.8 \pm 1.4$ \\
\cline { 2 - 3 } & C & $7.8 \pm 1.0$ \\
\hline \multirow{5}{*}{ S3 } & A & $12.4 \pm 1.9$ \\
\cline { 2 - 3 } & B & $8.8 \pm 1.5$ \\
\cline { 2 - 3 } & C & $7.3 \pm 1.1$ \\
\hline \multirow{3}{*}{ S4 } & A & $13.2 \pm 2.3$ \\
\cline { 2 - 3 } & B & $10.1 \pm 1.4$ \\
\cline { 2 - 3 } & C & $7.2 \pm 0.9$ \\
\hline
\end{tabular}




\subsubsection{Morphology of surface of pellets}

The surface morphology of the nearly spherical pellet before dissolution testing for samples S1A and S1C is presented in Fig. 6. For both, the surface was slightly rough; the MCC fibres, small crystals of Atn and some pieces of EC film (Fig. 6A, B $\rightarrow$ ) could be detected.

On contact with the dissolution medium, the particles that are immediately wetted change phase and then diffuse through the outer boundary layer exposed to the medium. The liquid gradually penetrates the porous network and the dissolved molecules then have to make an additional diffusional displacement within the porous pellets [32]. Dissolution leads to an increase in the size of the wetted pores (Fig. 6D $\rightarrow$ ), and the disparity of the wet zones generates a porosity gradient between the core and the periphery of the pellet, leading to changes in the structure and shape of the pellet. In the deeper layer, a piece of the EC film (Fig. 6G,I $\rightarrow$ ) or split film shreds can be seen (Fig. 6J $\rightarrow$ ). After dissolution, the surface was very rough and exhibited numerous cracks and pores caused by the diffusion of Atn in sample S1C rather than in sample S1A. Sample S1C displayed the formation of a film-like layer of MCC and partial melting of the EC at the temperature of production during extrusion. The jacket of the extruder was cooled, but the local increase of temperature was unavoidable.

In contrast, a smoother surface was observed on sample S3 than on sample S1. MCC and Atn particles were also seen for the untreated samples. After dissolution, large cracks and pores were formed, due to the liberation of the active agent. The film formation on sample S3 was more significant than that on sample S1; this may be due to the use of ethanol, in which EC is soluble. When ethanol was used in the second step of the wetting, the possibility of film formation on the surface of the particles was higher than when ethanol was applied initially. Of course, not only the selective appearance of the EC film formed from the solution, but also the "MCC film" and the film formed after hot melting must be considered. The presence of the additional film because of the application of ethanol explained the delayed dissolution of these samples. Its integrity (determined by the hardness) was therefore a very important ratelimiting parameter. 

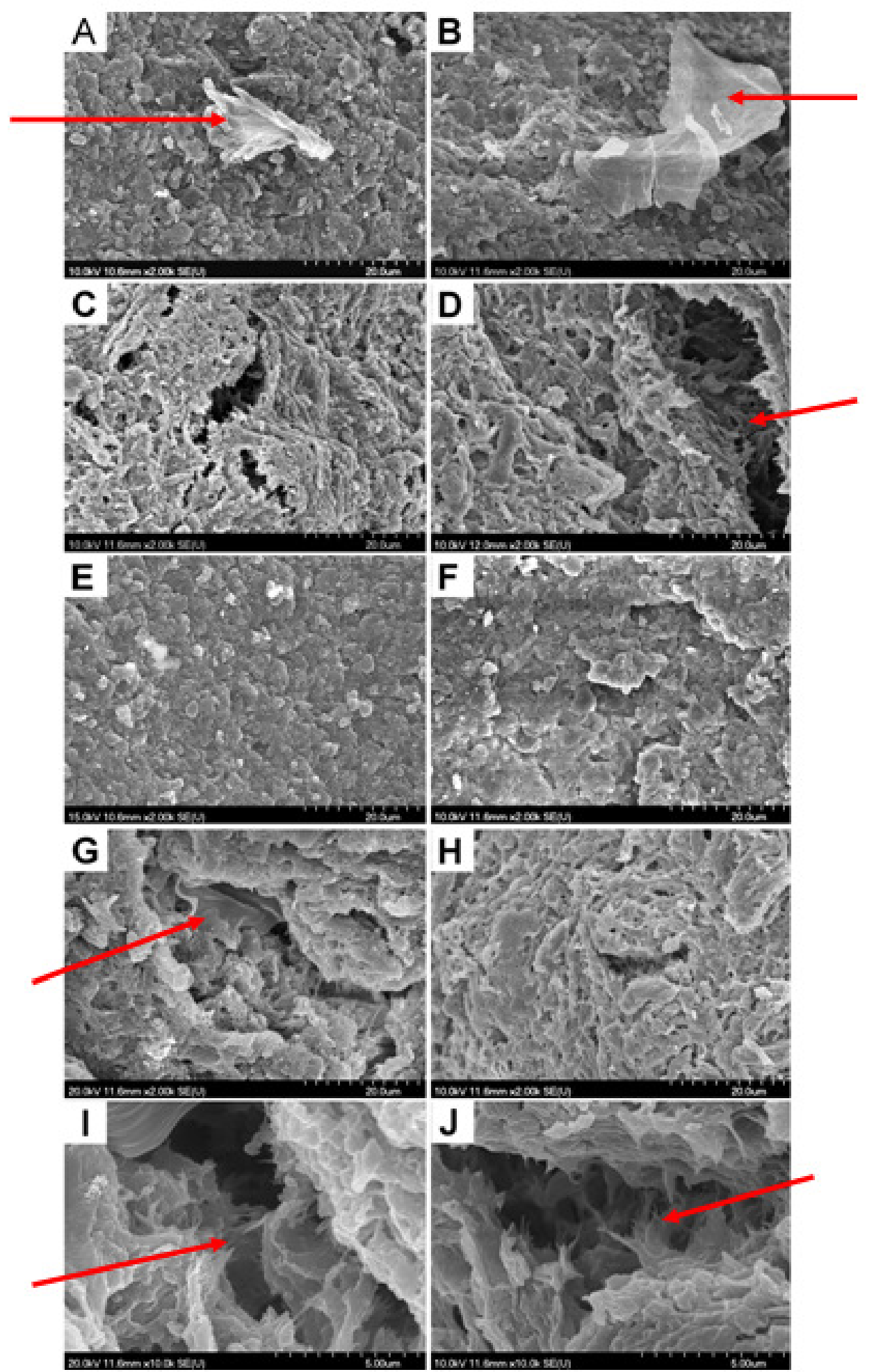

Fig. 6. Morphology of surface of pellets

A: S1A before dissolution. B: S1C before dissolution. C: S1A after dissolution. D: S1C after dissolution. E: S3A before dissolution. F: S3C before dissolution. G: S3A after dissolution. H: S1C after dissolution. I: S3A after dissolution. $\mathrm{J}$ : S1C after dissolution 


\subsection{Determination of optimum wetting liquid and operational parameters}

\subsubsection{Parameters of pellets}

During the process of extrusion/spheronization, determination of the main factors which can influence the preparation of pellets is very important. A $2^{3}$ full factorial design was utilized to optimize the circumstances applied during pelletization. The effects of the parameters of spheronization on the shape of the pellets and on the dissolution were tested. The effects of the nature of the wetting liquid applied for pelletization on the parameters of the final pellets were also examined. The spheronization was carried out according to the factorial design $\left(2^{3}\right)$ (Tables 5 and 6 ).

Table 5. Designation of samples

\begin{tabular}{|l|c|c|c|}
\hline Sample & $\begin{array}{c}X 1 \\
(\%)\end{array}$ & $\begin{array}{c}X 2 \\
(\mathrm{rpm})\end{array}$ & $\begin{array}{c}X 3 \\
(\mathrm{~min})\end{array}$ \\
\hline S1 & 16.3 & 750 & 10 \\
\hline S2 & 16.3 & 750 & 3 \\
\hline S3 & 16.3 & 1000 & 3 \\
\hline S4 & 16.3 & 1000 & 10 \\
\hline S5 & 0 & 750 & 10 \\
\hline S6 & 0 & 750 & 3 \\
\hline S7 & 0 & 1000 & 3 \\
\hline S8 & 0 & 1000 & 10 \\
\hline
\end{tabular}

Table 6. Values of factors

\begin{tabular}{|l|c|c|}
\hline Factor & Low level (-) & High level (+) \\
\hline Amount of ethanol $(X 1)$ & $0 \%$ & $16.3 \%$ \\
\hline Speed of spheronization $(X 2)$ & $750 \mathrm{rpm}$ & $1000 \mathrm{rpm}$ \\
\hline Duration of spheronization $(X 3)$ & $3 \mathrm{~min}$ & $10 \mathrm{~min}$ \\
\hline
\end{tabular}

The shapes of the pellets were very different for the different samples (Table 7 and Figs 7 and 8). Better results (lower values of the aspect ratio), i.e. nearly spherical products, were observed for the particles prepared with water. 


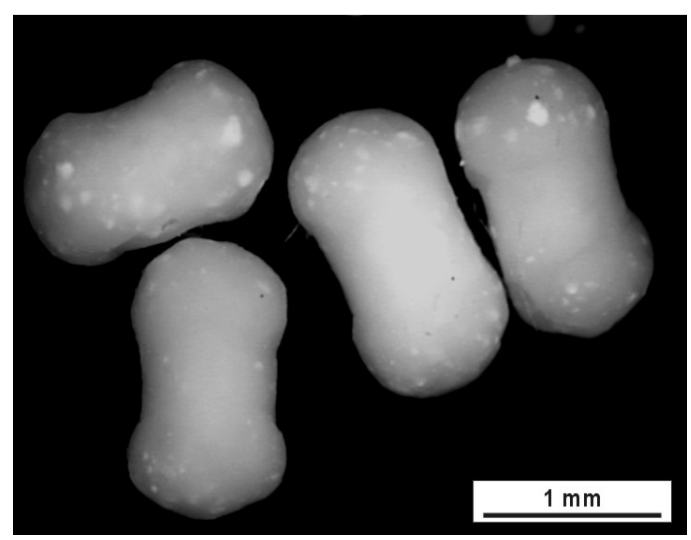

Fig 7. S2 particles

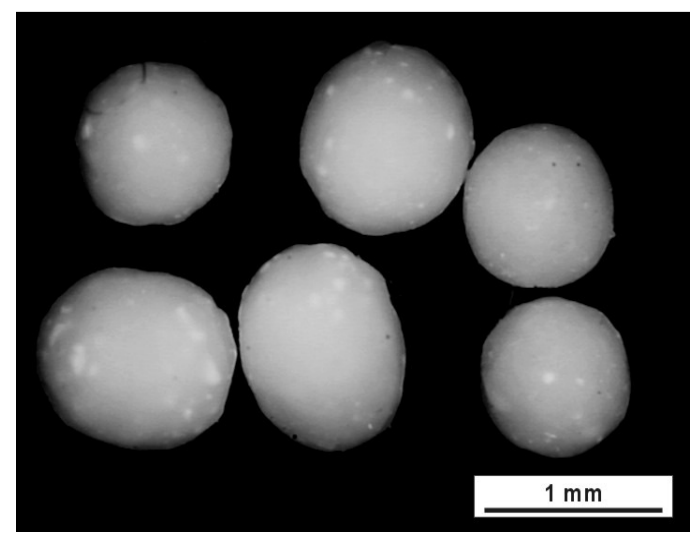

Fig 8. S8 particles

The situation was similar for the breaking hardness of the samples (Table 7). The evaluation of the process of pellet deformation involved not only determination of the breaking hardness, but also study of the deformation curve. The effect of the residual solvent on the mechanical properties of the spheres was neglected, because it was less than $0.5 \%$ for every sample.

The release of the active agent was sensitive to the nature of the kneading liquid. It was slower for the samples prepared with the mixture than for the samples prepared only with water (Table 7 samples S2 and S8, respectively). The curves were nearly linear, which is suitable for our aim, but a few samples exhibited a step in the first phase (burst effect) which must be avoided (Figure 9).

Table 7. Parameters of different pellets

\begin{tabular}{|l|c|c|c|c|c|c|}
\hline Sample & $\begin{array}{c}\text { Aspect } \\
\text { ratio }\end{array}$ & $\begin{array}{c}\text { Breaking } \\
\text { hardness } \\
(\mathrm{N})\end{array}$ & $\begin{array}{c}\text { Dissolution } \\
\text { at } 30 \mathrm{~min} \\
(\%)\end{array}$ & $\begin{array}{c}\text { Breadth } \\
(\mu \mathrm{m})\end{array}$ & $\begin{array}{c}\text { Length } \\
(\mu \mathrm{m})\end{array}$ & $\begin{array}{c}\text { Water } \\
\text { content } \\
(\%)\end{array}$ \\
\hline S1 & $1.54 \pm 0.28$ & $12.00 \pm 1.80$ & $37.55 \pm 2.54$ & $1491.4 \pm 142.6$ & $2303.6 \pm 484.1$ & 0.23 \\
\hline S2 & $1.75 \pm 0.40$ & $13.84 \pm 2.00$ & $31.46 \pm 5.30$ & $1430.3 \pm 118.2$ & $2519.1 \pm 643.3$ & 0.27 \\
\hline S3 & $1.61 \pm 0.32$ & $12.20 \pm 1.76$ & $36.91 \pm 3.72$ & $1439.6 \pm 93.0$ & $2326.9 \pm 502.1$ & 0.30 \\
\hline S4 & $1.38 \pm 0.19$ & $11.70 \pm 1.89$ & $51.06 \pm 2.43$ & $1478.8 \pm 69.2$ & $2035.8 \pm 281.9$ & 0.23 \\
\hline S5 & $1.33 \pm 0.16$ & $12.68 \pm 1.19$ & $66.70 \pm 5.64$ & $1450.7 \pm 64.7$ & $1928.3 \pm 274.8$ & 0.27 \\
\hline S6 & $1.55 \pm 0.27$ & $15.53 \pm 1.71$ & $67.97 \pm 2.50$ & $1430.3 \pm 102.5$ & $2235.3 \pm 465.1$ & 0.40 \\
\hline S7 & $1.49 \pm 0.25$ & $13.55 \pm 1.69$ & $62.27 \pm 6.81$ & $1429.3 \pm 88.7$ & $2138.1 \pm 339.8$ & 0.23 \\
\hline S8 & $1.17 \pm 0.09$ & $12.45 \pm 1.38$ & $54.13 \pm 5.66$ & $1455.7 \pm 50.2$ & $1705.3 \pm 155.9$ & 0.23 \\
\hline
\end{tabular}




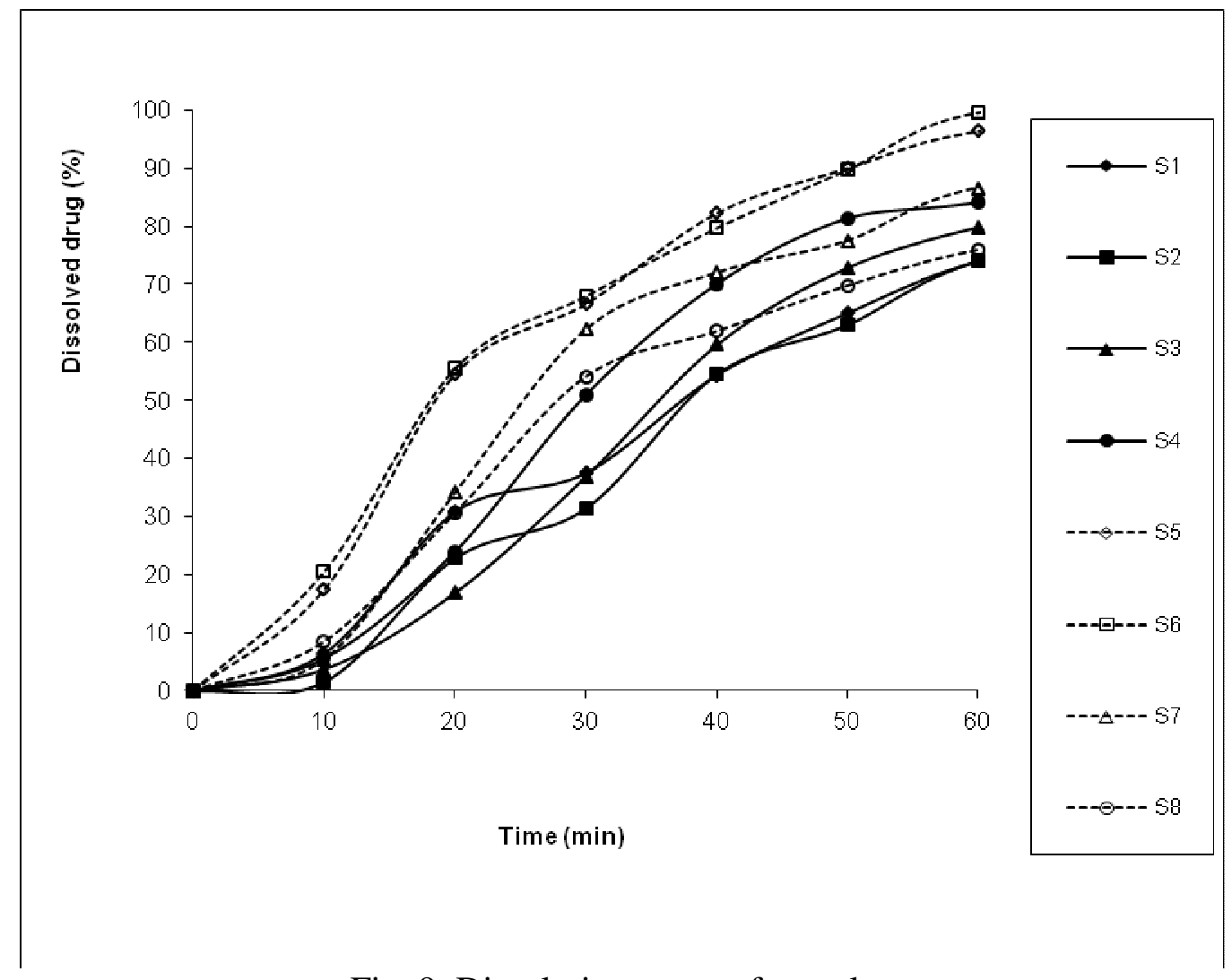

Fig. 9. Dissolution curve of samples

\subsubsection{Evaluation of effects of factors}

A linear approach was applied for the fitting, and the correlation coefficient $\left(R^{2}\right)$ was very good in every case (Table 8). The duration of spheronization was found to be a significant factor as concerns the shape of the pellets. Its negative value indicated that increase of the duration enhanced the shape of the particles. The presence of ethanol in the wetting liquid destroyed the efficiency of the spheronization process.

The duration of spheronization was also significant for the breaking hardness of the spheres. The two other factors were similarly important. Increase of the value of each of the factors caused a decrease in this parameter. It is known that the shape can modify the breaking hardness of different systems (mainly for tablets) [99,100]. In the present case, there was no obvious connection between the relevance of the factors for shape and breaking. Modification of the internal structure of the spheres must therefore be responsible for this phenomenon. Formation of a mass with plasticity appropriate for spheronization was not possible with the liquid containing ethanol. Suitable wetting of MCC could not be achieved with the liquid with this composition. The squeezing of this liquid during spheronization can also be quicker, and thus the formation of an adequate structure, which is formed by MCC, 
was also disturbed. This process was enhanced by high speed and the duration of spheronization, which can be the explanation of the negative sign of these factors.

Table 8. Values of coefficients

\begin{tabular}{|l|c|c|c|}
\hline Factor & $\begin{array}{c}\text { Coefficient for } \\
\text { aspect ratio }\end{array}$ & $\begin{array}{c}\text { Coefficient for } \\
\text { breaking hardness }\end{array}$ & $\begin{array}{c}\text { Coefficient for } \\
\text { dissolution }\end{array}$ \\
\hline Intercept & 1.4763 & 12.9913 & 50.6838 \\
\hline (1) Ethanol & 0.0913 & -0.5588 & -11.4413 \\
\hline (2) Speed & -0.0663 & -0.5188 & -0.2363 \\
\hline (3) Duration & $-0.1238^{*}$ & $-0.7888^{*}$ & 1.0313 \\
\hline 1 by 2 & -0.0113 & 0.0363 & 4.9738 \\
\hline 1 by 3 & 0.0113 & 0.2013 & 4.0263 \\
\hline 2 by 3 & -0.0163 & 0.3863 & -0.1738 \\
\hline $\mathrm{R}^{2}$ & 0.9973 & 0.9983 & 0.9731 \\
\hline
\end{tabular}

The presence of ethanol was most important for the dissolution at $30 \mathrm{~min}$. The effects of the other two factors were then negligible. In this case, the change in the shape can not be the sole explanation of this alteration. This observation can again be explained by the changes induced in the internal structure by the different wetting liquids. The wetting of EC was different, so its different matrix-forming behaviour was induced by the ethanol. The presence of this type of matrix generated a greater alteration in the dissolution than in the breaking hardness. The explanation of this phenomenon was that the relevance of MCC in the modification of dissolution from an inert matrix is less than that in the mechanical properties of spheres. The effect of EC can therefore predominate.

\subsection{Incorporation of alkalizing component}

Introduction of an alkalizing component is important to increase the $\mathrm{pH}$ at the site of absorption during parallel release of Atn from the matrix pellet in the micromilieu, to act as pore former, and also to lead to bridge formation during drying (crystal formation). In this work, $\mathrm{Na}_{2} \mathrm{HPO}_{4}$ or $\mathrm{Na}_{3} \mathrm{PO}_{4}$ was used as alkalizing component. 
Table 9. Compositions of samples

\begin{tabular}{|l|c|c|c|c|c|c|c|c|}
\hline \multirow{3}{*}{ Samples } & \multicolumn{9}{|c|}{ Powder } & \multicolumn{3}{|c|}{ Binding liquid } \\
\cline { 2 - 10 } & $\begin{array}{c}\text { Atn } \\
\%\end{array}$ & $\begin{array}{c}\text { MCC } \\
\%\end{array}$ & $\begin{array}{c}\mathrm{EC} \\
\%\end{array}$ & $\begin{array}{c}\mathrm{Na}_{2} \mathrm{HPO}_{4} \\
\%\end{array}$ & $\begin{array}{c}\mathrm{Na}_{3} \mathrm{PO}_{4} \\
\%\end{array}$ & $\begin{array}{c}\text { Water } \\
\%\end{array}$ & $\begin{array}{c}\text { Ethanol } \\
\%\end{array}$ & $\begin{array}{c}\text { Total } \\
\mathrm{ml}\end{array}$ \\
\hline P1 & 25 & 30 & 20 & 25 & - & 100 & - & 85 \\
\hline P2 & 25 & 30 & 20 & - & 25 & 100 & - & 85 \\
\hline P3 & 25 & 30 & 20 & 25 & - & 83.7 & 16.3 & 85 \\
\hline P4 & 25 & 30 & 20 & - & 25 & 83.7 & 16.3 & 85 \\
\hline P5 & 25 & 30 & 20 & 25 & - & 100 & - & 75 \\
\hline P6 & 25 & 30 & 20 & - & 25 & 100 & - & 75 \\
\hline P7 & 25 & 30 & 20 & 25 & - & 83.7 & 16.3 & 75 \\
\hline P8 & 25 & 30 & 20 & - & 25 & 83.7 & 16.3 & 75 \\
\hline
\end{tabular}

The shape parameters of the pellets were tested (Table 10). It can be seen from the data that the shapes of the different pellets were very different. The P2 Sample had the best aspect ratio (near to 1). This means that this sample, which was prepared with a higher amount of water and contained $\mathrm{Na}_{2} \mathrm{PO}_{4}$ had the best spherical form (Fig. 12). However, for the pellets that contained anhydrous $\mathrm{Na}_{2} \mathrm{HPO}_{4}$ the shape was not round and the surface was very rough (Fig. 10). The aspect ratio was a little better with a higher amount of wetting liquid.

Table 10. Shape parameters of pellets

\begin{tabular}{|l|c|c|c|}
\hline Sample & $\begin{array}{c}\text { Breadth } \\
(\mathrm{mm})\end{array}$ & $\begin{array}{c}\text { Length } \\
(\mathrm{mm})\end{array}$ & Aspect ratio \\
\hline P1 & $1.24 \pm 0.19$ & $1.44 \pm 0.42$ & $1.32 \pm 0.54$ \\
\hline P2 & $1.72 \pm 0.15$ & $1.88 \pm 0.16$ & $1.14 \pm 0.04$ \\
\hline P3 & $1.55 \pm 0.16$ & $1.95 \pm 0.37$ & $1.22 \pm 0.36$ \\
\hline P4 & $1.54 \pm 0.14$ & $1.68 \pm 0.22$ & $1.20 \pm 0.25$ \\
\hline P5 & $1.59 \pm 0.22$ & $2.73 \pm 0.70$ & $1.43 \pm 0.36$ \\
\hline P6 & $1.62 \pm 0.16$ & $1.99 \pm 0.33$ & $1.19 \pm 0.24$ \\
\hline P7 & $1.67 \pm 0.26$ & $3.40 \pm 0.94$ & $1.61 \pm 0.34$ \\
\hline P8 & $1.54 \pm 0.96$ & $1.92 \pm 0.24$ & $1.18 \pm 0.14$ \\
\hline
\end{tabular}

Figure 10. demonstrates the non-spherical form and the roughness of the surface of sample P1 and the higher magnification shows the sponge-like texture with many pores (Fig. 11). 


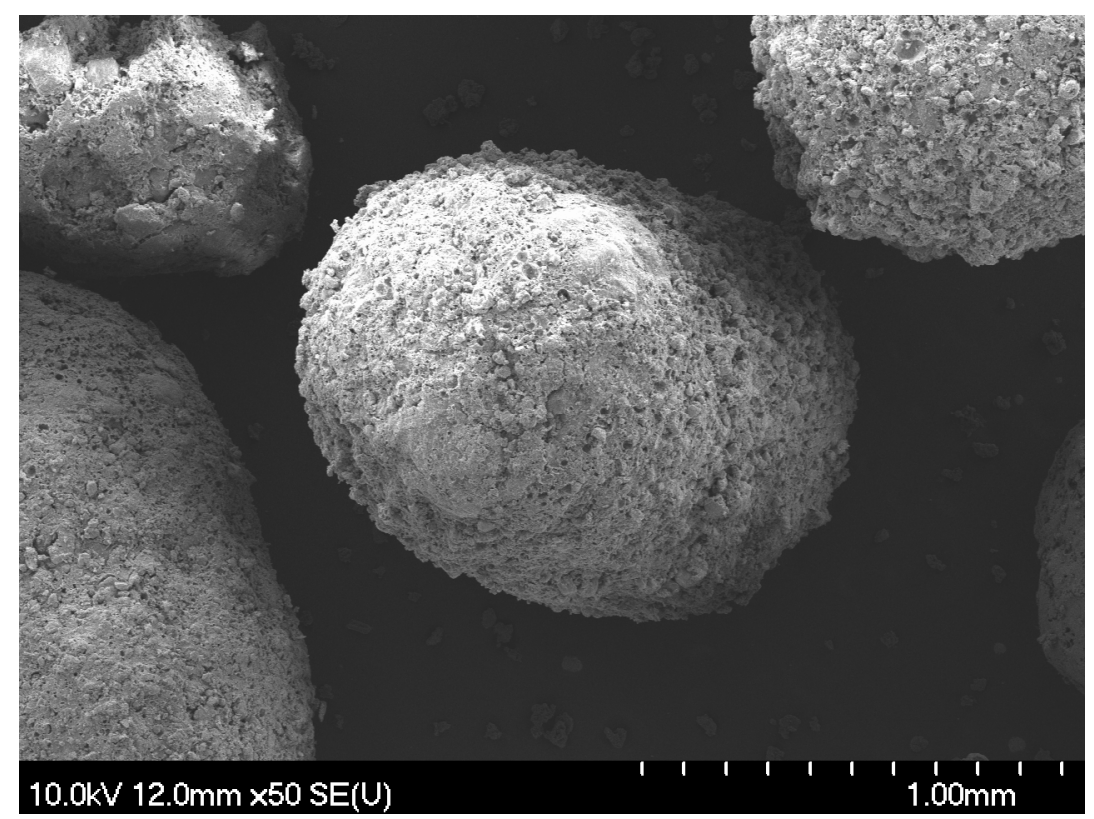

Fig. 10. Shape of sample P1 (SEM)

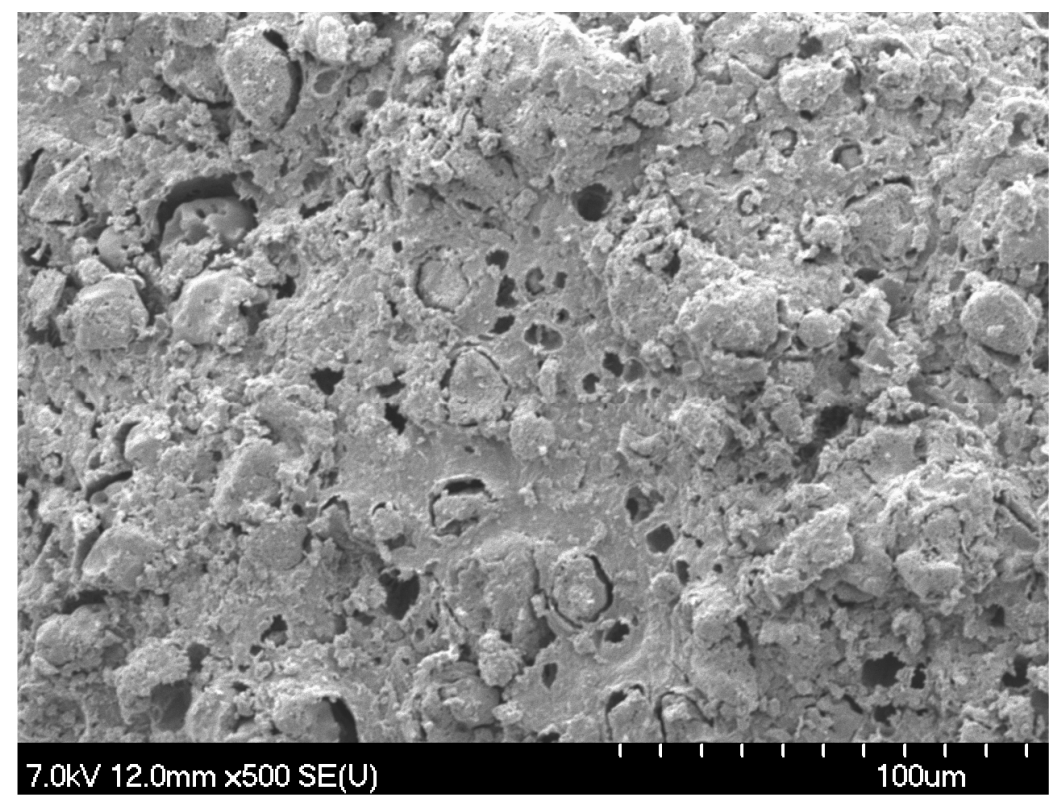

Fig. 11. Detail of surface of sample P1 (SEM)

The shape and surface of the pellets depend on the consistency of the mass. This phenomenon can influence the solubility of the components. Atn dissolves sparingly in water, but is soluble in alcohol. The water solubility of anhydrous $\mathrm{Na}_{2} \mathrm{HPO}_{4}$ and $\mathrm{Na}_{3} \mathrm{PO}_{4}$ is very good, but the latter dissolves in better water. These components are insoluble in alcohol. It is well known that the solubility of EC in alcohol is very good.

For the pellet containing MCC, the binding forces can be assigned to its 'crystallite-gel model' [97], MCC particles are broken down into smaller units and even partly into single crystals of colloidal size during granulation and extrusion in the presence of water. The resulting crystallites and porous particles form a coherent gel-like network (with a high 
fraction of an insoluble solid phase) and immobilize the liquid. Over a particular range of water, which relates to acceptable gel strength, extrusion and spheronization become possible.

In the samples which contained $\mathrm{Na}_{3} \mathrm{PO}_{4}$ and the wetting liquid was water alone in higher amount, the alkalizing component could take up the water from the powder mixture. This effect improves the plasticity of the wetted mass and enhances the spheronization, and is therefore a pellet structure- forming parameter which can determine not only the shape, but also the mechanical properties. During drying, rather fast recrystallization of $\mathrm{Na}_{3} \mathrm{PO}_{4}$ resulting in strong solid bridges between the other particles, e.g. MCC (P2, Fig. 12). The shape of the pellets was spherical, and the surface was smooth with some narrow or round pores, formed during drying. It could be seen at higher magnification that the texture of the pellet was very compact (Fig. 13) the hardness of these pellets was very high. On reduction of the water amount, the degree of recrystallization also decreased, but in spite of this the hardness was very good (Table 11).

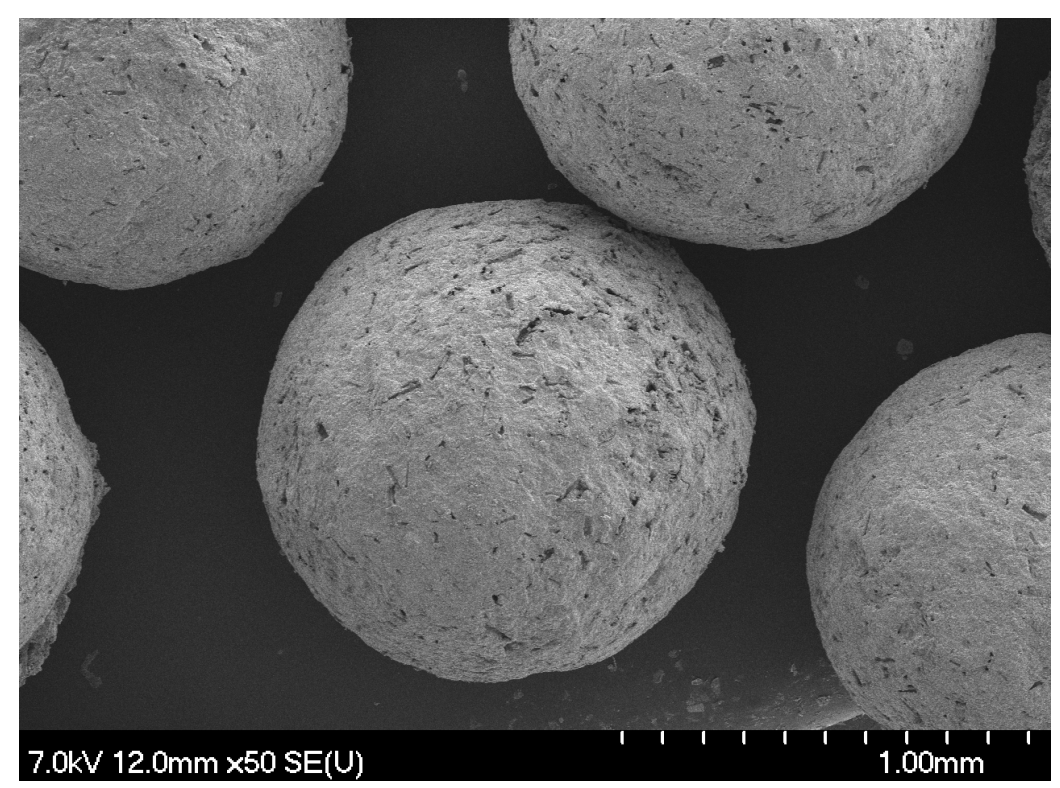

Fig. 12. Shape of P2 pellets (SEM) 


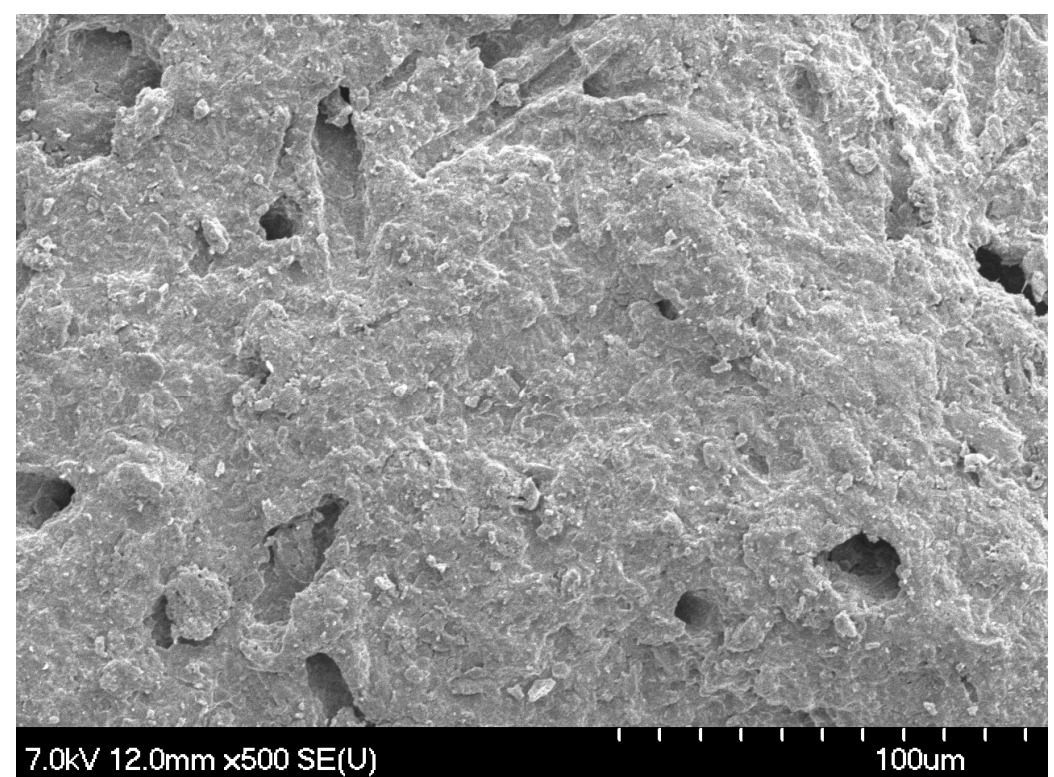

Fig. 13. Detail of surface of sample P2 (SEM)

Table 11. Breaking hardness of pellets

\begin{tabular}{|l|c|c|c|c|c|c|c|c|}
\hline Sample & P1 & P2 & P3 & P4 & P5 & P6 & P7 & P8 \\
\hline Breaking & 2.61 & 12.28 & 1.16 & 9.84 & 2.75 & 9.09 & 1.89 & 9.14 \\
hardness (N) & \pm 0.61 & \pm 2.03 & \pm 0.32 & \pm 1.82 & \pm 1.32 & \pm 1.78 & \pm 0.54 & \pm 3.09 \\
\hline
\end{tabular}

Through SEM investigation, the effect of ethanol on the texture of the pellets could be followed. A partial solution and fast recrystallization of Atn took place in the mass. A part of the EC also dissolved in ethanol and formed film pieces (Fig. 14). On reduction of the wetting liquid amount (and of course reduction of the ethanol in it), only smaller films could form and the recrystallization resulted in very small crystal aggregation (Fig. 15). Thus, the solid bonds in the texture weakened to a smaller degree. Higher pores could be seen in the texture and the hardness was a little less in comparison with P2 (Fig. 12). However, it can be seen from the data Table 3 that the pellets containing $\mathrm{Na}_{3} \mathrm{PO}_{4}$ had very high hardness. Decrease of the amount of water or the use of a water/ethanol mixture as wetting liquid reduced the hardness, but to only a small degree (P4, P6 and P8). 


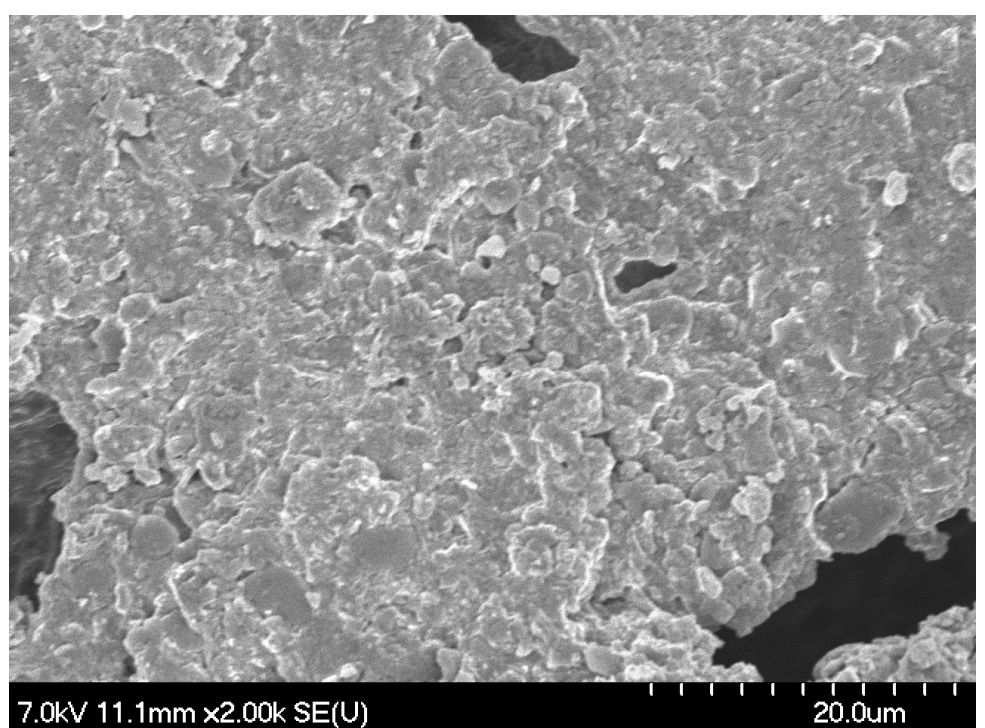

Fig. 14. Detail of surface of sample P4 (SEM)

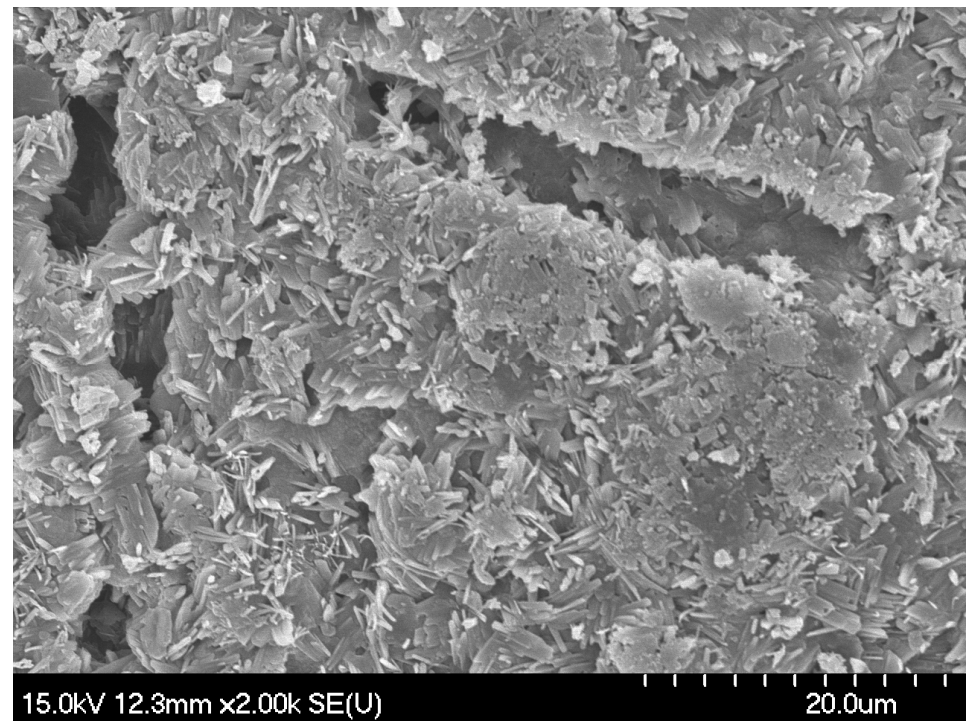

Fig. 15. Detail of surface of sample P8 (SEM)

The texture of the pellets containing anhydrous $\mathrm{Na}_{2} \mathrm{HPO}_{4}$ was generally looser. It can be seen in Fig. 10 that these pellets prepared with water alone were non-spherical and were very rough (P1). The higher magnification shows that the partial solution and fast recrystallization resulted in a sponge-like texture with numerous pores (Fig. 11). This explains the small value of the breaking hardness. In the case of a smaller amount of water the degree of recrystallization decreased and many small bonds could be observed among the small crystals and particles in the texture (Fig. 16, P5). 


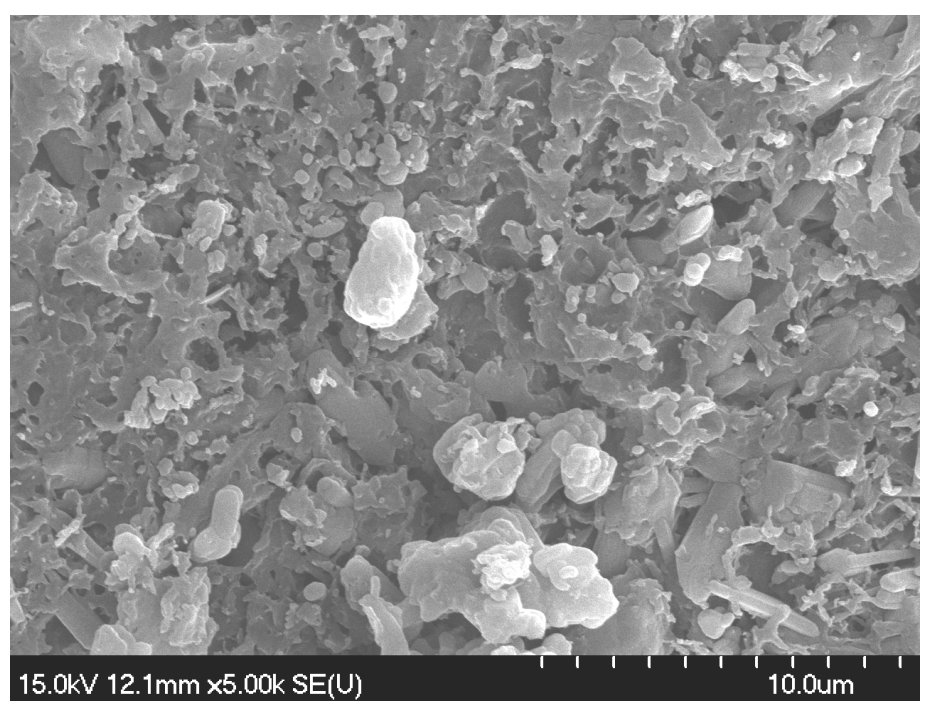

Fig. 16. Detail of surface of sample P5 (SEM)

In the presence of ethanol, Atn and EC could be dissolved, resulting in recrystallized Atn aggregates (Fig. 16) and variously sized pieces of EC film, as can be seen in the texture of P3 (Fig. 17), in comparison with the SEM photo of bulk Atn (Fig. 18), which demonstrates the crystal habit of Atn.

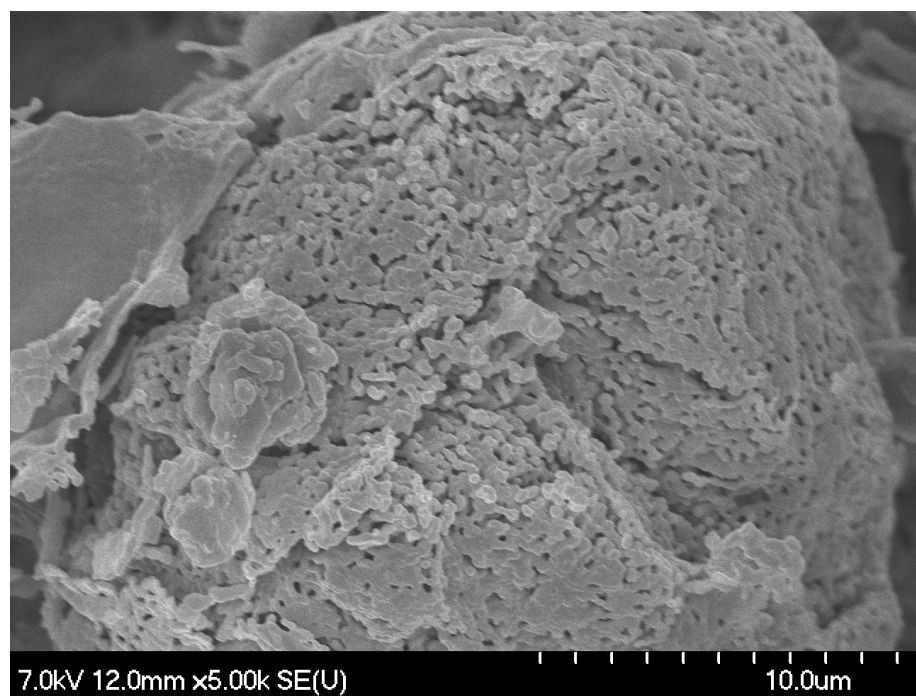

Fig. 17. Detail of surface of sample P3 (SEM) 


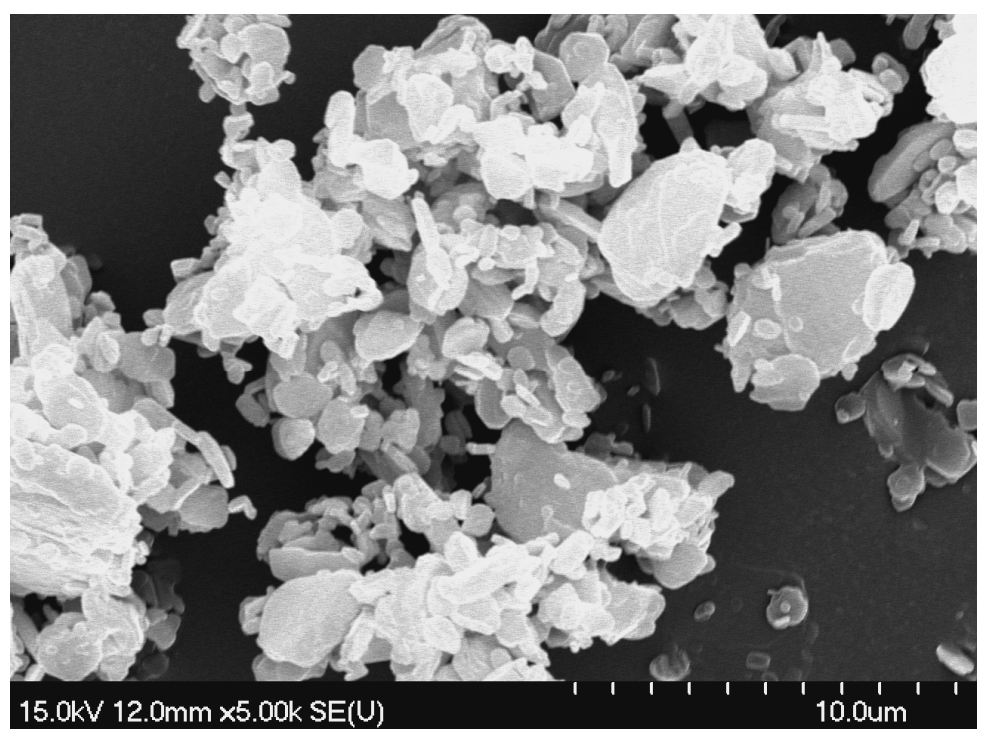

Fig. 18. Atn crystals (SEM)

Many small crystals could be seen among the larger irregular-shaped crystals a lot of this habit is in agreement with the habit of small recrystallized particles (Fig. 10). On decrease of the wetting liquid the sponge-like matrix perdominated, which was created by EC film with a few Atn crystals spread on its surface (Fig. 19, P7).

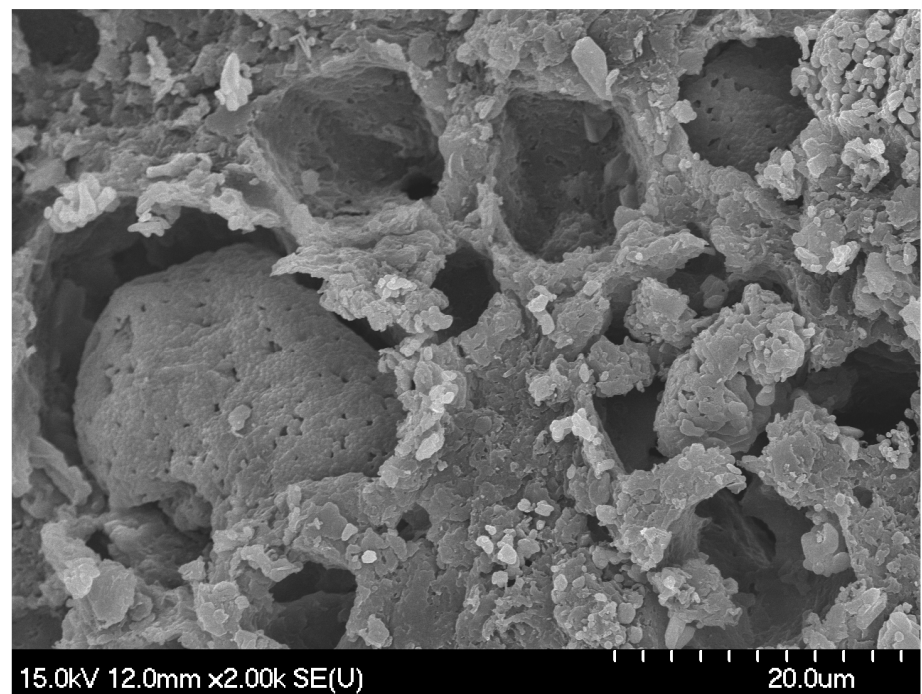

Fig. 19. Detail of surface of sample P7 (SEM)

As concerns the pellets containing anhydrous $\mathrm{Na}_{2} \mathrm{HPO}_{4}$ it can be stated that the hardness of these pellets were practically independent from wetting liquid (P1, P3, P5 and P7).

It was mentioned above that from the aspect of suitable dissolution and bioavailability the role of the $\mathrm{pH}$ in the intestine is very important. For this reason, the effect of the alkalizing component on the $\mathrm{pH}$ in the micromilieu was studied. 
In Tables 12 and 13, the effect of the dissolved alkalizing components on the $\mathrm{pH}$ change can be followed. It can be seen that in water the $\mathrm{pH}$ increased very quickly in the micromilieu and this effect lasted for $1 \mathrm{~h}$. After $10 \mathrm{~min}$, the $\mathrm{pH}$ value was more than $10 \mathrm{in}$ every case, independently of the type of pellets. After 20 min some difference could be seen, but it can generally be stated that, in the case of $\mathrm{Na}_{3} \mathrm{PO}_{4}$ containing pellets, the $\mathrm{pH}$ was more than 10 and decreased progressively during time. The duration of the alkaline $\mathrm{pH}$ value was best for the pellets $\mathrm{P} 2$.

Table 12. Influence of pellets on $\mathrm{pH}$ of solution

\begin{tabular}{|l|c|c|c|c|c|c|c|c|}
\hline Sample & \multicolumn{7}{|c|}{$\mathrm{pH} /$ time (min) in water } \\
& 0 & 10 & 20 & 30 & 40 & 50 & 60 & 70 \\
\hline P1 & 5.81 & 10.60 & 10.05 & 7.31 & 6.25 & 6.05 & 5.98 & 5.84 \\
\hline P2 & 5.82 & 10.67 & 10.36 & 10.08 & 9.60 & 8.60 & 6.50 & 5.87 \\
\hline P3 & 5.58 & 10.65 & 8.92 & 6.80 & 6.64 & 6.01 & 5.90 & 5.56 \\
\hline P4 & 5.67 & 10.65 & 10.40 & 8.70 & 6.34 & 6.05 & 6.32 & 5.84 \\
\hline P5 & 5.81 & 10.70 & 9.80 & 9.40 & 9.00 & 7.90 & 6.50 & 5.90 \\
\hline P6 & 5.77 & 10.64 & 10.31 & 9.68 & 8.25 & 7.80 & 6.80 & 6.02 \\
\hline P7 & 5.81 & 10.70 & 9.80 & 9.40 & 9.00 & 7.90 & 6.50 & 5.90 \\
\hline P8 & 5.77 & 10.64 & 10.31 & 9.68 & 8.25 & 7.80 & 6.80 & 6.02 \\
\hline
\end{tabular}

Table 13. Influence of pellets on $\mathrm{pH}$ of solution

\begin{tabular}{|l|c|c|c|c|c|c|c|c|}
\hline Sample & \multicolumn{7}{|c|}{$\mathrm{pH} /$ time (min) in buffer } \\
& 0 & 10 & 20 & 30 & 40 & 50 & 60 & 70 \\
\hline P1 & 6.8 & 7.83 & 7.81 & 7.76 & 7.74 & 7.71 & 6.95 & 6.83 \\
\hline P2 & 6.8 & 7.50 & 7.32 & 7.13 & 7.01 & 6.92 & 6.89 & 6.83 \\
\hline P3 & 6.8 & 7.80 & 6.93 & 6.85 & 6.84 & 6.83 & 6.81 & 6.81 \\
\hline P4 & 6.8 & 7.59 & 7.22 & 6.93 & 6.90 & 6.86 & 6.84 & 6.81 \\
\hline P5 & 6.8 & 7.70 & 7.52 & 7.41 & 7.21 & 7.00 & 6.86 & 6.83 \\
\hline P6 & 6.8 & 7.71 & 7.58 & 7.45 & 7.21 & 7.02 & 6.90 & 6.86 \\
\hline P7 & 6.8 & 7.65 & 7.18 & 7.03 & 7.00 & 6.97 & 6.90 & 6.86 \\
\hline P8 & 6.8 & 7.68 & 7.41 & 7.32 & 7.20 & 7.00 & 6.87 & 6.85 \\
\hline
\end{tabular}

The effect of the dissolved alkalizing components could be seen in buffer solution too, but to a smaller degree. After $10 \mathrm{~min}$, the $\mathrm{pH}$ in the micromilieu had increased from 6.8 to 7.5-7.8. The duration of this effect was best in the cases of $\mathrm{P} 1$ and $\mathrm{P} 2$, where the granulation liquid was water alone. These results suggest the better dissolution of Atn.

The in vitro dissolution tests showed generally revealed a tendency to sustained release and the alkalizing micromilieu enhanced the dissolution of Atn from the matrix. The dissolution profiles showed the sigmoid shape, which characterized the sustained release preparations (Fig. 20). 


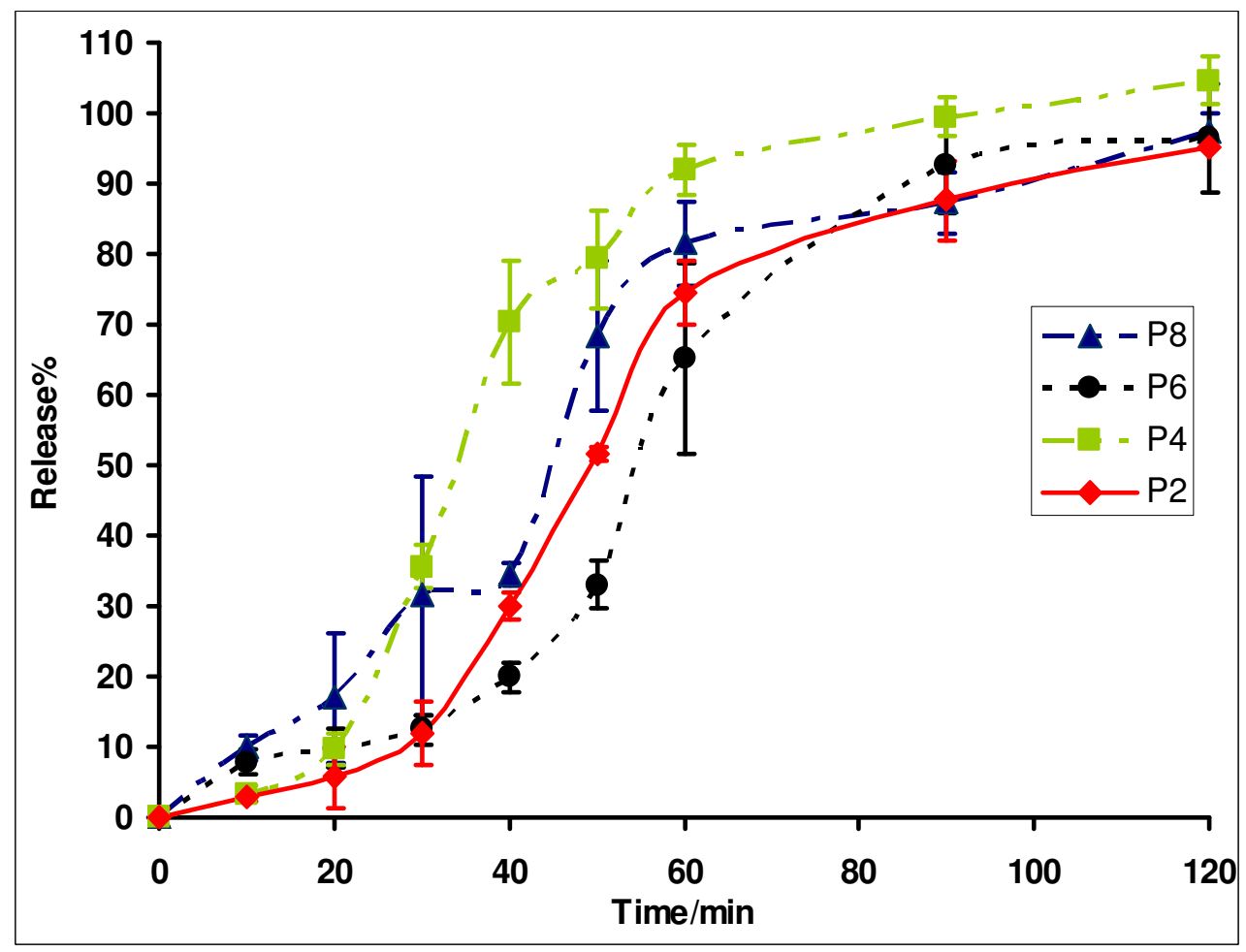

Fig. 20. Dissolution profiles of pellets containing $\mathrm{Na}_{3} \mathrm{PO}_{4}$

However the dissolution from the samples containing anhydrous $\mathrm{Na}_{2} \mathrm{HPO}_{4}$ was very uneven (P1, P3, P5 and P7) and the deviation was too high (sometimes more than 20\%). The reason lay in the very loose texture with large pores and the too weak bonds. Because of this, these pellets were omitted from the further experiments.

The dissolution from the pellets containing $\mathrm{Na}_{3} \mathrm{PO}_{4}$ (P2, P4, P6 and P8) was better, and the deviation (SE) was smaller (Fig. 20). The best deviation was observed for the P2 and P4 pellets, when the amount of wetting liquid was used in higher. For the P6 and P8 pellets when the amount of wetting liquid was lower; the deviation was higher. These results are in accordance with the texture of the pellets. The most uniform dissolution was achieved in the case of sample $\mathrm{P} 2$, when the alkalizing agent was $\mathrm{Na}_{3} \mathrm{PO}_{4}$ and the binding liquid was a higher amount of water. The texture of this pellet was very compact and the hardness was very high.

\subsection{Preparation of pellets with optimum parameters and their coating}

As regards the final dosage form, the pellets containing Atn should be coated with intestine-soluble polymer. In accordance with the morphological observation and the hardness of pellets, the dissolution results indicated that the P2 pellets had the best composition, suitable for the development of the final dosage form.

For the coating process, $\mathrm{P} 2$ pellets were prepared again and other compositions too, without and with $\mathrm{Na}_{3} \mathrm{PO}_{4}$ (Table 14). 
Table 14. Preparation of pellets with different contents

\begin{tabular}{|l|c|c|c|c|c|c|c|c|c|}
\hline Samples & \multicolumn{6}{|c|}{ Sample contents } & \multicolumn{3}{c|}{ Coating } \\
\cline { 2 - 10 } & $\begin{array}{c}\text { Atn } \\
\%\end{array}$ & $\begin{array}{c}\mathrm{Na}_{3} \mathrm{PO}_{4} \\
\%\end{array}$ & $\begin{array}{c}\mathrm{MCC} \\
\%\end{array}$ & $\begin{array}{c}\text { EC } \\
\%\end{array}$ & $\begin{array}{c}\text { Lactose } \\
\%\end{array}$ & $\begin{array}{c}\text { Water } \\
\mathrm{ml}\end{array}$ & $\begin{array}{c}\text { Opadry } \\
\%\end{array}$ & $\begin{array}{c}\text { Acryl-EZE } \\
\text { MP } \\
\% \text { w/w }\end{array}$ & Dye \\
\hline B0 & 25 & 25 & 30 & 20 & - & 85 & 3 & 17 & \\
\hline B1 & 25 & 25 & 30 & - & 20 & 85 & - & - & - \\
\hline B2 & 25 & - & 30 & 20 & 25 & 85 & - & 20 & \\
\hline B3 & 25 & - & 30 & - & 45 & 851 & - & 20 & \\
\hline
\end{tabular}

The optimum properties of the pellets (round shape, hardness and dissolution) were obtained for samples B0, B2 and B3, but not for B1, which failed in the granulation step, producing a very sticky plastic mass, resisting extrusion and hence spheronization, this being due to the interaction that occured between the alkalizing component and lactose. The reason is that lactose is incompatible with many APIs. It forms Schiff bases with primary amino groups (present in Atn) and alkaline materials promote the decomposition.

Subcoating materials have been widely used to prevent interactions between an API and an enteric coating. Our formula B0 contained a high amount of a strong alkalizing agent, which influenced the dissolution of the Atn earlier than required. The alkalizing component has very good water solubility and could dissolve in the water component of the coating dispersion, so that some of it could migrate into the polymer film. Accordingly, it was necessary to protect the core before the functional coating. The protective layer used was HPMC (Opadry clear) (mass 3\% w/w). After drying of the protective layer (10 min), the coating was continued with the functional, $\mathrm{pH}$-dependent polymer dispersion (Acryl EZE MP). There was no need for a subcoating layer for samples B2 and B3, because there was no alkalizing component in their compositions.

The results of dissolution testing showed, that the Atn could not dissolve in the gastric juice, but a small difference in dissolution could be seen in the intestinal juice profiles (Fig.21). 


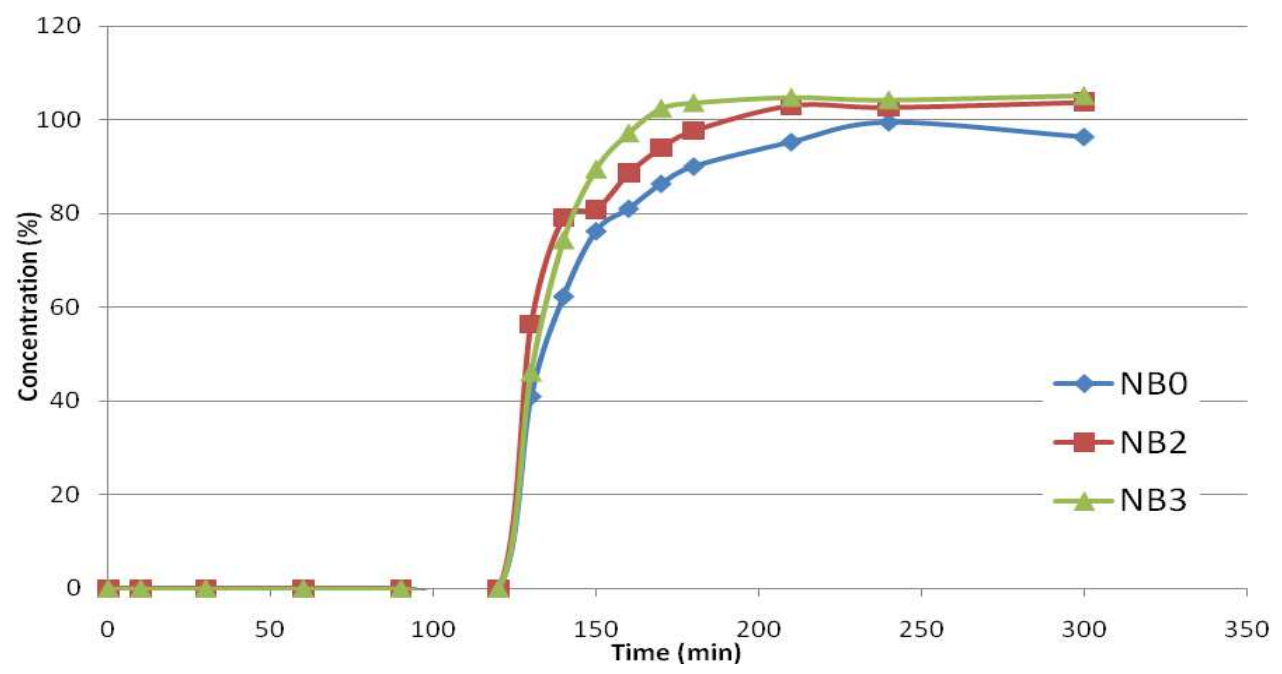

Fig 21. Dissolution profile of coated pellets in fluid of $\mathrm{pH} 1.2$ for first $2 \mathrm{~h}$, then continued in buffer of $\mathrm{pH} 6.8$

The total amount of Atn dissolved from sample B3 during 150 min. Only a moderate dissolution was observed in the case of B2. For B0, the rate of dissolution decreased to a small degree and the total Atn dissolved during 250-300 min. These pellets were doublecoated pellets.

B0 pellets were also prepared in another experiment without a subcoating layer. The samples were coated only with the functional polymer layer. The dissolution profiles of the two kinds of pellets are compared in Fig. 22. It can be seen that in the gastric juice, about $30 \%$ of the Atn dissolved.

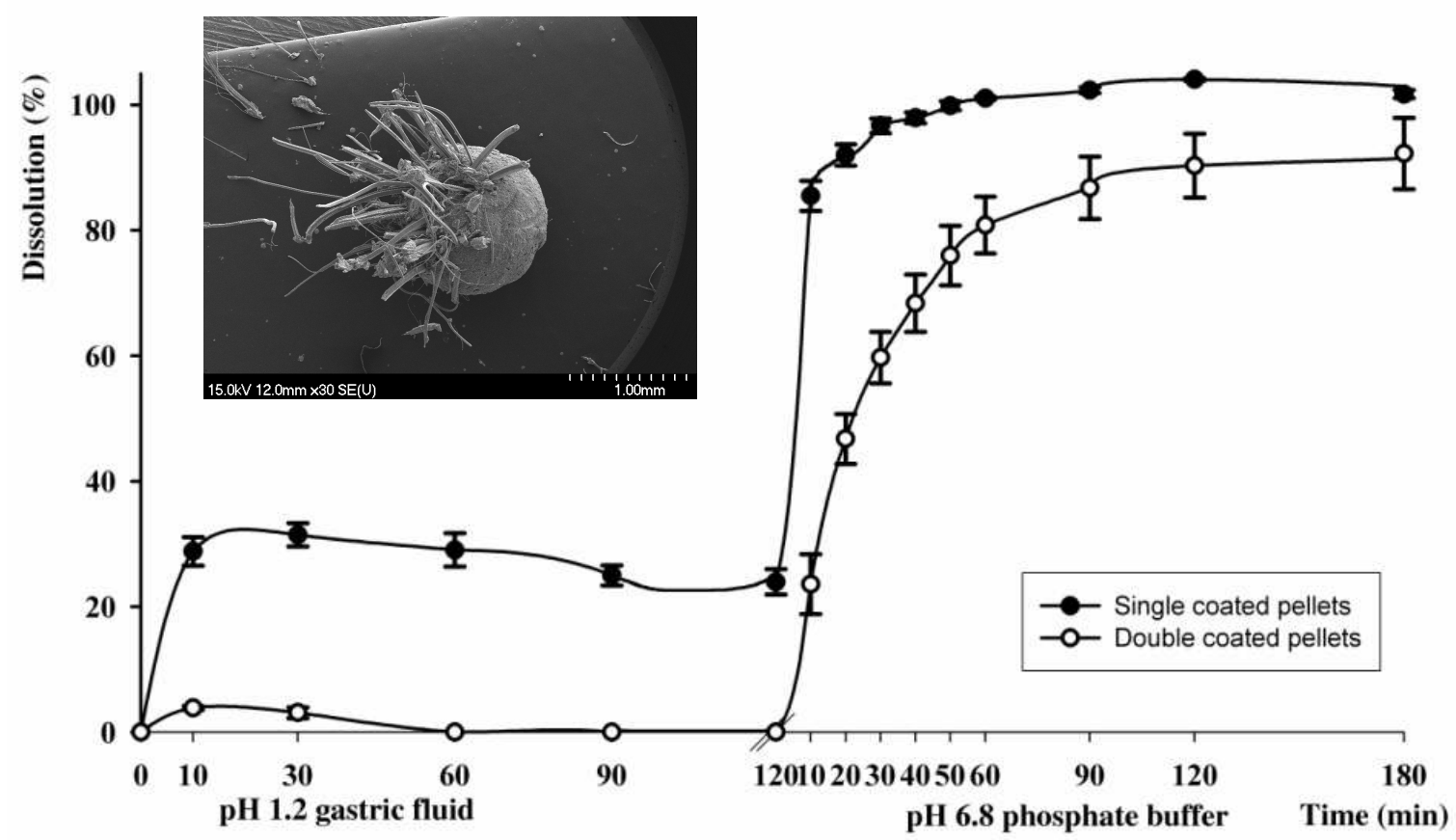

Fig. 22. Dissolution profiles of Atn from single-coated and double-coated pellets 
In the SEM picture, some longer and shorter needle crystals were observed on the surface of the uncoated pellets (Fig. 22, inset). These crystals developed through the recrystallization of $\mathrm{Na}_{3} \mathrm{PO}_{4}$ during drying, which can easily break off from the surface and lead to pore formation on the surface. This is the other reason for the higher dissolution (about 30\%) in the gastric juice, in spite of the gastric juice-resistant coating (Aryl EZE MP).

The shape parameter was tested before coating, and after coating and double coating for B0, which exhibited more round pellets due to the smoothing of the surface of the pellets during the coating process (Table 15).

Table 15. Hardness and shape parameters of pellets before and after coating

\begin{tabular}{|l|c|c|c|c|}
\hline Sample & Aspect ratio & $\begin{array}{c}\text { Breaking } \\
\text { hardness } \\
(\mathrm{N})\end{array}$ & $\begin{array}{c}\text { Breadth } \\
(\mu \mathrm{m})\end{array}$ & $\begin{array}{c}\text { Length } \\
(\mu \mathrm{m})\end{array}$ \\
\hline B0 & $1.10 \pm 0.04$ & $12.28 \pm 2.0$ & $1776 \pm 139.1$ & $1954.8 \pm 148.7$ \\
\hline B2 & $1.07 \pm 0.03$ & $7.87 \pm 1.1$ & $1720.3 \pm 92.9$ & $1868.1 \pm 119$ \\
\hline B3 & $1.07 \pm 0.03$ & $6.14 \pm 3.1$ & $1711.4 \pm 123.9$ & $1842.6 \pm 136.1$ \\
\hline coated & \multicolumn{5}{|l}{} \\
\hline B0 & $1.04 \pm 0.03$ & - & $1832.9 \pm 147.2$ & $1997.8 \pm 182.9$ \\
\hline B2 & $1.06 \pm 0.05$ & - & $1757.4 \pm 140.5$ & $1882.2 \pm 205.2$ \\
\hline B3 & $1.07 \pm 0.03$ & - & $1880.6 \pm 102.3$ & $2015.4 \pm 118$ \\
\hline
\end{tabular}

The hardness of the pellet was found to be $12.29 \pm 2.03 \mathrm{~N}$, which is very good for coating. The alkalizing component could promote the wettability of the powder mixture. This effect improved the plasticity of the wetted mass and enhanced the spheronization, and determined not only the mechanical properties, but also the shape of the pellets. During drying, rather fast recrystallization of the $\mathrm{Na}_{3} \mathrm{PO}_{4}$ occurred, resulting in strong solid bridges with the MCC particles. Table 15 reveals that the pellets before coating were spherical, with an aspect ratio close to 1 . This means that these samples were very good for coating. It can be seen from the data that the shape of the pellets after coating was also rounded, and in the case of B0 the roundness was better. This means that they were smoothed by the covering layer of the coating polymer (Fig. 22).

The dissolution test revealed that Atn could not dissolve in the gastric juice from the double-coated pellets because the protective layer closed the pores of the core and did not allow the migration of any component in the outer layer. However, the total amount of Atn could dissolve in the phosphate buffer $(\mathrm{pH}=6.8)$ during $3 \mathrm{~h}$ (Fig. 22). The dissolution revealed a typical delayed release profile: the dissolution of the drug started only after $2 \mathrm{~h}$. This means that the protecting layer on the surface of the core was working efficiently. The 
aqueous solution could not pass into the core and the alkalizing component could not migrate into the Acryl EZE film. In the phosphate buffer, both coating layers and hence the alkalizing component could dissolve, and the alkalizing micromilieu enhanced the dissolution of Atn from the matrix pellet. Figures 23-25 illustrate the cross-section of the double-coated pellet.

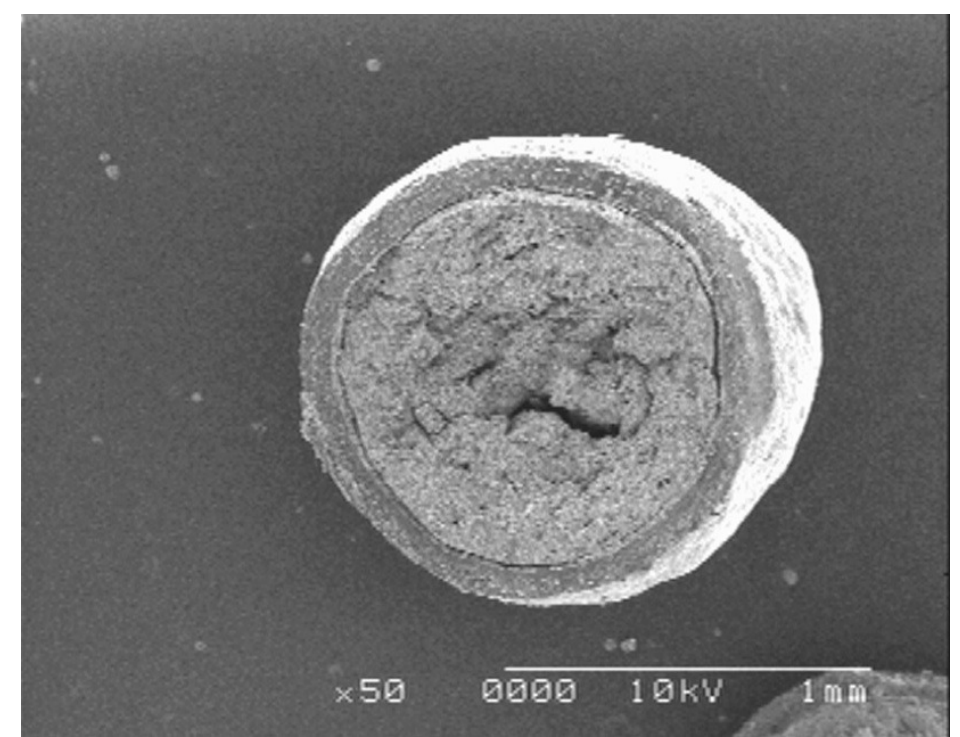

Fig. 23. Cross-section of the double-coated pellet (SEM). Magn.: 50x

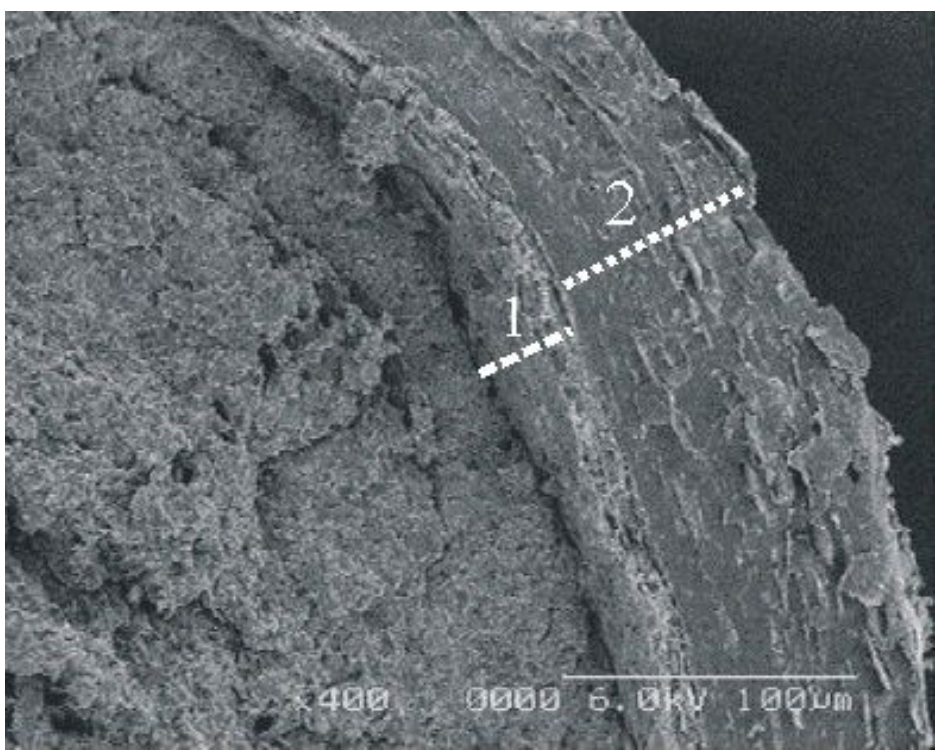

Fig. 24. Cross-section of the double-coated pellet (SEM). Magn.: 400x 1: protective layer; 2 : functional layer 


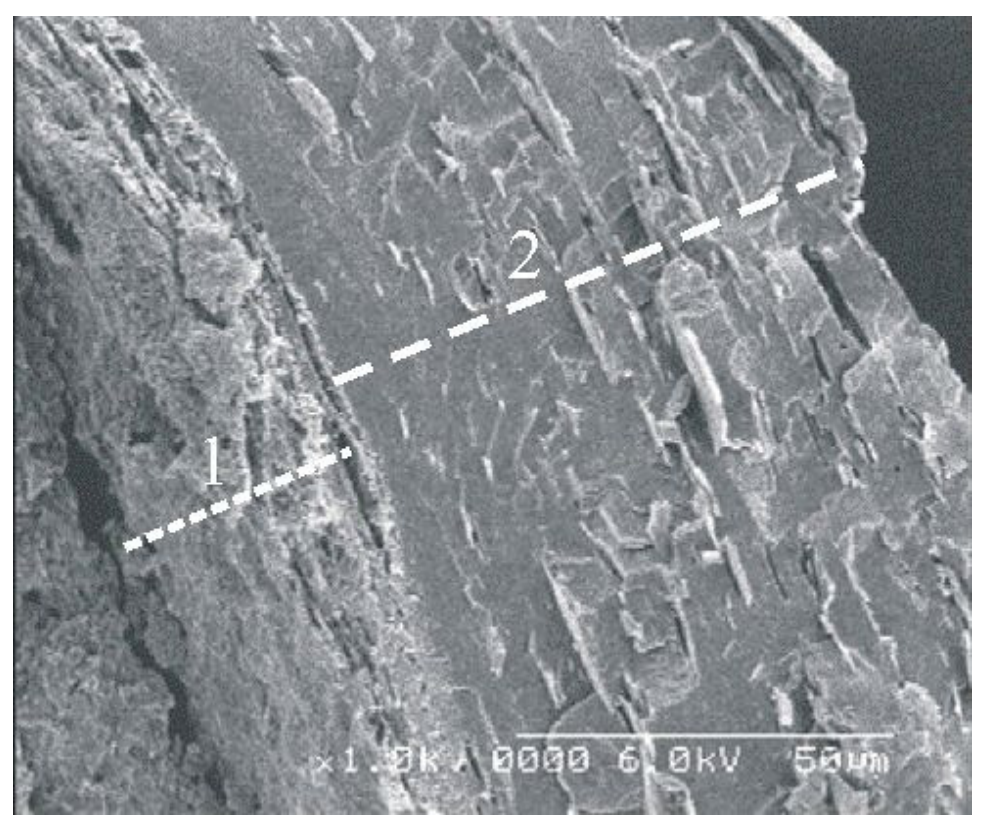

Fig. 25. Cross-section of the double-coated pellet (SEM). Magn.: 1000x

1: protective layer; 2 : functional layer

At low magnification (Fig. 24), the spherical form and a relative large pore in the middle of the pellet could be observed, but around the pore the matrix was rather compact. At higher magnification (Fig. 25), the coating layer was clearly visible and the two polymer layers were distinguishable (Fig. 26); it could be observed that the adherence between the two kinds of polymer was very strong.

\subsection{In vivo evaluation of bioavailability of Atenolol}

Dissolution tests (in vitro) are applied as a tool with which to predict drug product performance in vivo. The dissolution curves in Fig. 21 demonstrate that the Atn could not dissolve in the gastric juice, but there was a small difference in dissolution profiles in the intestinal juice (Fig. 21). The total amount of Atn dissolved from sample B3 during 150 min. Moderate dissolution took place in the case of B2. The dissolution of Atn decreased slightly in the case of B0, and the total amount of Atn dissolved during 250-300 min. These pellets were double-coated pellets.

The complete release of Atn was achieved from all samples in vitro, but the dissolution conditions do not fully represent the environment in the gastrointestinal tract, and the results can be interpreted only on an empirical basis. In the in vitro tests, the solubility of the Atn was determined. Measurement of the absorption of Atn under these circumstances is not possible.

The compositions of the media were fine-tuned according to the phase of digestion both in the stomach and in the intestines. These and other physiological and chemical factors 
can dramatically affect drug solubility and dissolution in the upper small intestine, and hence the rate of absorption. To study the bioavailability of Atn in rats after oral administration of $6.0 \mathrm{mg}$ in capsules, the plasma was collected and analysed by HPLC. The HPLC curves revealed a high peak level in the plasma and hence the area under the curve for sample B0 was much greater than those for samples B2 and B3 (Fig. 26).

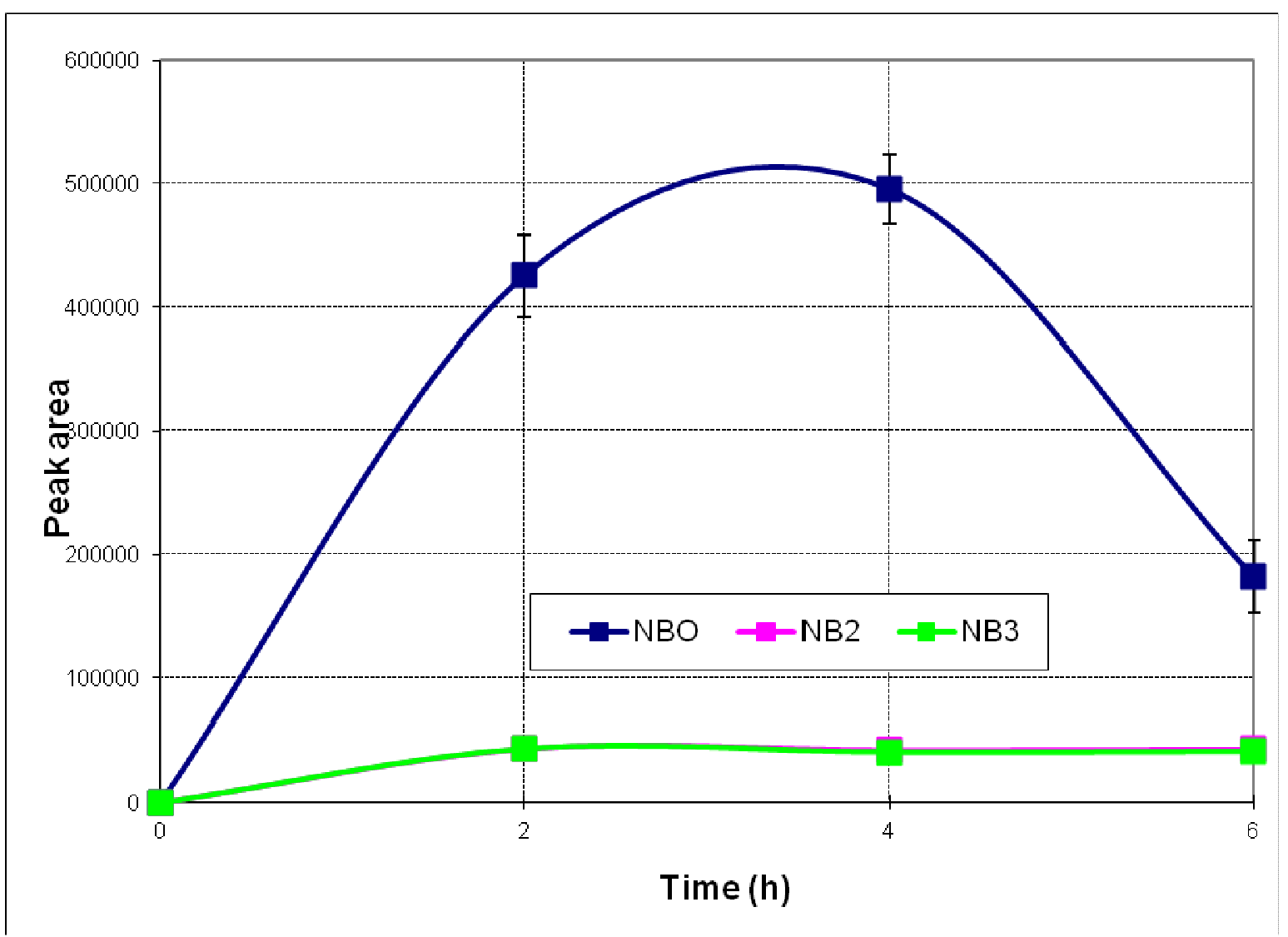

Fig. 26. HPLC plasma concentration - time curve of pellets in rats

It may be concluded that the alkalizing component enhances the absorption of Atn from matrix pellets prepared by an extrusion/spheronization technique and containing EC as matrixformer.

The plasma concentration for NB2 and NB3 was $34 \mu \mathrm{g} / \mathrm{ml}$ after $2 \mathrm{~h}$, up to $8 \mathrm{~h}$. while NBO was changed by time. After $2 \mathrm{~h}$ it was $323 \mu \mathrm{g} / \mathrm{ml}$, after $4 \mathrm{~h}$ it was $376 \mu \mathrm{g} / \mathrm{ml}$, and after 6 $\mathrm{h}$ it was $138 \mu \mathrm{g} / \mathrm{ml}$. 


\section{SUMMARY}

The main aim of this work was the formulation of matrix pellets containing Atn by means of extrusion/spheronization with a view to increasing its bioavailability.

On the basis of this study, it can be concluded that water or different water/ethanol combinations are appropriate for the formulation of pellets from powder mixtures containing different ECs, MCC and Atn.

The dissolution profiles of the samples prepared with water followed first-order kinetics. For the samples where the granulating liquid also contained ethanol, the dissolution changed, and a lag time was observable. In this case, the ethanol dissolves the EC, and in the drying period an EC film was formed around the drug particles, this layer delaying dissolution. Finally, it may be stated that the application of ethanol in the wetting liquid led to a decrease in the dissolution in the first phase, but also caused a reduction in the breaking strength of the pellets. The formation and the structure of the pellets differed for the sample prepared with liquid containing ethanol.

The use of a factorial design confirmed that significant effects were exerted not only by the operational parameters, but also by the nature of the wetting liquid. Besides the shape, the internal structure of the pellets was changed by the optimum combination of factors, as indicated by the mechanical properties of the spheres and the dissolution of the active agent. The presence of ethanol in the liquid caused different degrees of wetting in the different components of the powder mixture. This step of our work is of help in the design of pellets containing an alkalizing component for increase of the bioavailability of the active agent.

In order to enhance the bioavailability of Atn from pellets, alkalizing components (anhydrous $\mathrm{Na}_{2} \mathrm{HPO}_{4}$ and $\mathrm{Na}_{3} \mathrm{PO}_{4}$ ) were used. It can be concluded that the shape parameters of the pellets were best when $\mathrm{Na}_{3} \mathrm{PO}_{4}$ was the alkalizing component. As concerns the wetting liquid, the higher amount of water alone resulted in pellets with the best compact texture, with a smooth surface and considerable hardness. MCC formed a coherent gel-like network and the water-soluble $\mathrm{Na}_{3} \mathrm{PO}_{4}$ underwent rapid recrystallization, resulting in strong solid bridges with the other particles. It is clear that the alkalizing component is able to increase the $\mathrm{pH}$ in the micromilieu. This effect is presumably manifested in the intestine too.

In the in vitro experiments, the dissolution release complied with the texture of the pellets and the effect of $\mathrm{pH}$. The most uniform and total release of Atn was observed for the 
sample containing $\mathrm{Na}_{3} \mathrm{PO}_{4}$ and prepared with water alone, which also has a very good hardness and a round shape, and which is suitable for coating and further investigation.

The results of the experiments revealed that the pellets prepared by extrusion and spheronization were spherical and had high strength. This product was suitable for coating. The in vitro dissolution tests demonstrated that the alkalizing component promoted the dissolution of the total amount of Atn from the pellets at $\mathrm{pH} 6.8$, but use of a protective polymer layer was necessary before the functional polymer coating. This double-coated pellet is an excellent product which is suitable for filling into capsules.

Finally, the alkalizing component enhances the absorption of Atn from the matrix pellet prepared by the extrusion/spheronization technique.

\section{PRACTICAL USEFULNESS}

My experimental work has allowed the following conclusions:

- Factorial design is very important for the evaluation of factors and parameters during pellet preparation processing.

- Extrusion/spheronization is a very good method with which to prepare pellets containing EC, MCC and Atn with optimum properties.

- EC polymers can be used to form a matrix for Atn in the preparation of pellets with MCC extrusion/spheronization technique.

- MCC is necessary for the formulation of good mass as it influence the formulation behaviour.

- Atn as an API can be formulated in modified release matrix pellets with a view to enhancing bioavailability.

- Alkalizing components act as pore-formers which improve the dissolution of Atn and also increase the $\mathrm{pH}$ of the micromilieu due to their ability to cause parallel release of Atn and enhance its absorption. 


\section{REFERENCES}

1. Felipe J.O., Varum, H.A., Merchant, A.W.B., Oral modified-release formulations in motion: The relationship between gastrointestinal transit and drug absorption, 2010 Int. J. Pharm. 395, 26-36

2. Bechgaard H., Hagermann N.G., Controlled-release multi-units and single unit doses. A literature review, 1978. Drug Dev. Ind. Pharm. 4, 53-67.

3. Bodmeier R.,Tableting of coated pellets, 1997. Eur. J. Pharm. Biopharm. 43, 1-8.

4. Krämer J., Blume H., Biopharmaceutical aspects of multiparticulates, in: I. GhebreSellassie (Ed.), Multiparticulate Oral Drug Delivery, Marcel Dekker Inc., New York, 1994, pp. 307-332.

5. Ghebre-Sellassie I., Pellets: a general overview, in: I. Ghebre-Sellassie (Ed.), Pharmaceutical Pelletization Technology, Marcel Dekker Inc.,New York, 1989, pp. 1-13.

6. Ghebre-Sellassie I., Knoch A., Pelletization techniques, in: J. Swarbrick, J.C. Boylan(Eds.), Encyclopedia of Pharmaceutical Technology, Marcel Dekker Inc, New York, 2002, pp. 2067-2080.

7. Umprayn K., Chitropas P., Amarekajorn S., Influence of process variables on physical properties of the pellets using an extruder and spheronizer, 1999. Drug Dev. Ind. Pharm. $25,45-61$

8. Law M.F., and Deasy P.B., Use of hydrophilic polymers with microcrystalline cellulose to improve extrusion-spheronization, 1998.Eur. J. Pharm. Biopharm. 45, 57-65,

9. Vervaet C, Baert L and Remon JP. Extrusion-spheronization: literature review, 1995, Int. J. Pharm. 116, 131-146.

10. Bussemer T., Otto I., Bodmeier R., Pulsatile drug-delivery systems, 2001. Crit. Rev. Ther. Drug Carrier Sys. 18, 433-458.

11. Bussemer T., Bodmeier R., Review of pulsatile drug delivery, 2001. Am. Pharm. Rev. 424.

12. Lemmer B., Chronopharmacokinetics: implications for drug treatment, 1999. J. Pharm. Pharmacol. 51, 887-890.

13. Ritschel W.A., Forusz H., Chronopharmacology: a review of drugs studies, 1994 Meth. Find. Exp. Clin. Pharmacol. 16, 57-75.

14. Bechgaard H., Hagermann N.G., Controlled-release multi-units and single unit doses. A literature review, 1978 Drug. Dev. Ind. Pharm. 4, 53-67. 
15. Bergeron M., Laurin P., Tawashi R., Effect of particle morphology in selecting pharmaceutical excipients, 1986. Drug Dev. Ind. Pharm. 12, 915-926.

16. Barret, P.J: The shape of rock particles, a critical review, Sedimentology 27(1980) 291303.

17. Gamlen, M.J., Pellet manufacture for controlled release, 1985.Manuf. Chem. 56, 55-59.

18. Chambliss, W.G., Conventional and specialized coating pans. Pharmaceutical Pelletization Technology, Dekker, New York, 1989, pp. 15-38.

19. Olsen, K.W., Fluid bed equipment. Pharmaceutical Pelletization Technology, Dekker, New York, 1989, pp. 39-70.

20. Gamlen, M.J., Eardley, C., Continuous extrusion using a Baker Perkins MP50 (Multipurpose) extruder. Drug Dev. Ind. Pharm., 12 (1986) 1701-1713.

21. Otsuka M., Gao J., Matsuda Y., Effect of amount of added water during extrusionspheronization process on pharmaceutical properties of granules, 1994. Drug Dev Ind Pharm. 20, 2977-2992.

22. Newton J.M., Extrusion/spheronization. In: Chulia D, Deleuil M, Pourcelot Y, editors. Powder technology and pharmaceutical processes. Amsterdam: Elsevier; 1994.

23. Noche C., Barochez B.H., Brossard C., Horvath S., Cuiné A., Optimising the manufacturing process for controlled release pellets, 1994. Pharm Techn Eur 4, 39-46.

24. Fekete R., Effect of the formulation parameters on the characteristics of pellets, 1998. Drug Dev Ind Pharm 24,1073-1076.

25. Santos H., Veiga F., Pina M., Podczeck F., Sousa J., Physical properties of chitosan pellets produced by extrusion/ spheronization: Influence of formulation variables, 2002. Int J Pharm. 246, 153-169.

26. De Brabander C., Vervaet C., Remon J.P., Development and evaluation of sustained release mini-matrices prepared via hot melt extrusion, 2003. J Control Release. 89, 235247.

27. De Brabander C, Vervaet C, Van Bortel L, Remon JP. Bioavailability of ibuprofen from hot-melt extruded mini-matrices, 2004. Int J Pharm. 271, 77-84.

28. Agrawal A.M., Howard M.A., Neau S.H., Extruded and spheronized beads containing no microcrystalline cellulose: influence of formulation and process variables, 2004. Pharm. Dev. Technol. 9, 197-217.

29. Tho I., Sande S.A., Kleinebudde P., Pectinic acid, a novel excipient for production of pellets by extrusion/spheronization: preliminary studies, 2002. Eur. J. Pharm. Biopharm. 54, 95-99. 
30. Basit A.W., Newton M., and Lacey L.F., Formulation of ranitidine pellets by extrusionspheronization with little or no microcrystalline cellulose, 1999. Pharm. Dev. Technol. 4, 499-505.

31. Newton J.M., Extrusion and extruders. In: Swarbrick J., Boylan J.C., (Eds.). Encyclopedia of Pharmaceutical Technology. Marcel Dekker Inc., New York and Basel, pp. 1220-1236, 2002.

32. Shah R.D., Kabadi M., Pope D.G., Augsburger L.L., Physicomechanical characterization of the extrusion-spheronization process. 2. Rheological determinants for successful extrusion and spheronization, 1995. Pharm. Res. 12, 496-507.

33. Berggren and J., Alderborn G., Effect of drying rate on porosity and tableting behaviour of cellulose pellet, 2001. Int. J. Pharm. 227, 81-96.

34. Habib Y.S., and R.F., Shangraw R.F., Effect of different drying techniques on the physico-mechanical properties of beads containing microcrystalline cellulose produced by extrusion and spheronization. Pharm. Res. 14, 5-14, 1997.

35. Dyer A.M., Khan K.A., Aulton M.E., Effects of the drying method on mechanical and release properties of pellets prepared by extrusion-spheronization, 1994. Drug Dev. Ind. Pharm. 20, 3045-3068.

36. Kleinebudde P., Shrinking and swelling properties of pellets containing MCC and low substituted hydroxy propyl cellulose. Part I. Shrinking properties, 1994. Int. J. Pharm. $109,209-219$.

37. Bataille B., Ligarski K., Jacob M., Thomas C., Duru C., Study of the influence of spheronization and drying conditions on the physico-chemical properties of neutral spheroids containing Avicel PH101 and lactose, 1993. Drug Dev. Ind. Pharm. 19, 653671.

38. Sonaglio D., Bataille B., Ortigosa C., Jacob M., Factorial design in the feasibility of producing Microcel MC 101 pellets by extrusion/spheronization, 1995. Int. J. Pharm. 115, $53-60$.

39. Fielden K.E., Newton J.M., Rowe R.C., The influence of lactose particle size on spheronization of extrudate processed by a ram extruder, 1992. Int. J. Pharm. 81, 205224.

40. Ye Z-W., Rombout P., Remon J.P., Vervaet C., Van Den Mooter G., Correlation between the permeability of metoprolol tartrate through plasticized isolated ethylcellulose/hydroxypropylmethylcellulose films and drug release from reservoir pellets, 2007. Eur. J. Pharm. Biopharm. 67, 485-490. 
41. Iyer E.M., Augsburger L.L., and Parikh D.M., Evaluation of drug layering and coating: effect of process mode and binder level, 1993. Drug Dev. Ind. Pharm. 19, 981-998.

42. Wallace J.W., Cellulose derivatives and natural products utilized in pharmaceutics. In: Swarbrick J., Boylan J. C. (Eds.). Encyclopedia of Pharmaceutical Technology. Marcel Dekker Inc., New York and Basel. pp, 319-339, 2002.

43. Verhoeven E., De Beer T.R.M., Van den Mooter G., Remon J.P., and Vervaet C.V., Influence of formulation and process parameters on the relelase characteristics of ethylcellulose sustained-release mini-matrices, 2008. Eur. J. Pharm. Biopharm. 69, 312319.

44. Huang J., Wigent R.J., Bentzley C.M., Schwartz J.B., Nifedipine solid dispersion in microparticles of ammonio methacrylate copolymer and ethylcellulose binary blend for controlled drug delivery: Effect of drug loading on release kinetics, 2006. Int. J. Pharm. $319,44-54$.

45. DeMerlis C.C., Schoneker D.R., Borzelleca J.F., A subchronic toxicity study in rats and genotoxicity tests with an aqueous ethylcellulose dispersion, 2005. Food Chem. Toxicol. 43, 1355-1364.

46. Naelapää K., Veski P., Kristensen H.G., Bertelsen P., Coating of potassium chloride with ethylcellulose aqueous dispersion-effects of processing parameters on film formation and film properties, 2007. Eur. J. Pharm. Sci. 32, S20.

47. Erkoboni, K.A. Extrusion/spheronisation, in: Parikh D., (Ed.), Handbook of Pharmaceutical Technology. (Marcel Dekker Inc., New York), 1997.

48. McGinity, J.W., Aqueous Polymeric Coatings for Pharmaceutical Dosage Forms, 2nd. (Marcel Dekker, New York), 1997.

49. Dashevsky, A., Kolter, K., Bodmeier, R., pH-independent release of a basic drug from pellets coated with the extended release polymer dispersion Kollicoat SR 30 D and the enteric polymer dispersion Kollicoat MAE 30 DP, 2004. Eur. J. Pharm. Biopharm. 58, $45-50$

50. Khan, M.Z.I., Prebeg, Z., Kurjakovic, N., A pH-dependent colon targeted oral drug delivery system using methacrylic acid copolymers: I. Manipulation of drug release using Eudragit L100-55 and Eudragit S100 combinations, 1999. J. Control. Release. 58: 215222.

51. Lecomte, F., Siepmann, J., Walther, M., MacRae, R.J., Bodmeier, R., Polymer blends used for the coating of multiparticulates: comparison of aqueous and organic coating techniques, 2004. Pharm. Res. 21, 882-890. 
52. Garcia-Arieta, A., Torrado-Santiago, D., Torrado, J.J., Comparative study of aqueous and organic enteric coatings of chlorpheniramine maleate tablets, 1996. Drug Dev. Ind. Pharm. 22, 579-585.

53. Lorck, C.A., Grunenberg, P.C., Juenger, H., Laicher, A., Influence of process parameters on sustained-release theophylline pellets coated with aqueous polymer dispersions and organic solvent-based polymer solutions, 1997. Eur. J. Pharm. Biopharm. 43, 149-157.

54. Wesseling, M., Bodmeier, R., Drug release from beads coated with an aqueous colloidal ethylcellulose dispersion, Aquacoat, or an organic ethylcellulose solution, 1999.Eur. J.Pharm. Biopharm. 47, 33-38.

55. Moustafine, R.I., Kabanova, T.V., Kemenova, V.A., Van den Mooter, G., Characteristics of interpolyelectrolyte complexes of Eudragit E100 with Eudragit L100, 2005. J. Con. Rel 103, 191- 198.

56. Guo, H.X., Heina"ma"ki, J., Yliruusi, J., Amylopectin as a subcoating material improves the acidic resistance of enteric-coated pellets containing a freely soluble drug, 2002. Int. J. Pharm. 235, 79-86.

57. Chang, R.K., A comparison of rheological and enteric properties among organic solutions, ammonium salt aqueous solutions, and latex systems of some enteric polymers, 1990. Pharm. Technol. 10, 62-70.

58. Bianchini, R., Resciniti, M., Vecchio, C., Technology evaluation of aqueous enteric coating systems with and without insoluble additives, 1991. Drug Dev. Ind. Pharm. 17, 1779-1794.

59. Obara, S., Maruyama, N., Nishiyama, Y., Kokubo, H., Dry coating: an innovative enteric coating method using a cellulose derivative, 1999. Eur. J. Pharm. Biopharm. 47, 51-59.

60. Felton, L.A., Haase, M.M., Shah, N.H., Zhang, G., Infeld, M.H., Malick, A.W., McGinity, J.W., Physical and enteric properties of soft gelatin capsules coated with Eudragit L 30 D55, 1995. Int. J. Pharm. 113, 17-24.

61. Bozdag, S., Calis, S., Sumnu, M., Formulation and stability evaluation of entericcoated omeprazole formulations, 1999. STP Pharma Sci. 9, 321-327

62. Crotts, G., Sheth, A., Twist, J., Ghebre-Sellassie, I., Development of an enteric coating formulation and process for tablets primarily composed of a highly water-soluble, organic acid, 2001. Eur. J. Pharm. Biopharm. 51, 71-76.

63. Bruce, L.D., Koleng, J.J., McGinity, J.W., The influence of polymeric subcoats and pellet formulation on the release of chlorpheniramine maleate fromenteric coated pellets, 2003. Drug Dev. Ind. Pharm. 29, 909-924. 
64. Griffin, J.P., The Textbook of Pharmaceutical Medicine 6th Ed., New Jersey: BMJ Books.

65. Factors Influencing the Measurement of Bioavailability, Taking Calcium as a Model. 2001. Robert P., Heaney. J., Nutr. 131,1344S-1348S

66. Semple K.T., Doick K.J., Jones K.C., Burauel P., Craven A., Harms H., 2004. Environ. Sci. Technol. 38 (12) 228A-231A

67. Shargel L., Yu A.B., Applied biopharmaceutics \& pharmacokinetics (4th ed.). New York: McGraw-Hill. 1999.

68. Heaney R.P., Factors Influencing the Measurement of Bioavailability, Taking Calcium as a Model, 2001. J. Nutr. 131, (4 Suppl) 1344S-1348S.

69. Briggs G.G., Freeman R.K., Yaffe S.J., Drugs in Pregnancy and Lactation, Fourth edition, Williams and Wilkins, Baltimore, 1994.

70. Niebyl J.R., Teratology, Drugs in Pregnancy and Lactation, in: T. Scott (Ed.), Danforth's Obstetrics and Gynecology, 6th ed., Lipincott, Philadelphia, 1990.

71. Fitzgerald J.D., The biological and clinical effects of atenolol (Tenormin) a cardioselective-antagonist, in: M.E. Goldberg (Ed.), Pharmacological and Biochemical Properties of Drug Substances, vol. II, American Pharmaceutical Association Press, Washington DC, 1979.

72. Thompson F.D., Review of clinical pharmacology of atenolol, Proc. R. Soc. Med. 70, (Suppl. 5) 5-8. 1977.

73. Tabacova S.A., Kimmel C.A., Atenolol: pharmacokinetic/dynamic aspects of comparative developmental toxicity, 2002. Reprod. Toxicol. 16, 1-7.

74. Rigby J.W., Scott A.K., Hawksworth G.M., Petrie J.C., A comparison of the pharmacokinetics of atenolol, metoprolol, oxprenolol and propranolol in elderly hypertensive and young healthy subjects, Br, 1985. J. Clin. Pharmacol. 20, 327-331.

75. Jiang J.J., Tian, L. Huang, Y.L. Li, Y.S. Xu, L.Pharmacokinetic and pharma- codynamic characteristics of aranidipine sustained-release, enteric-coatedtablets in healthy Chinese men: a Phase I, randomized, open-label, single- and multiple-dose study, 2008 Clin Ther. $301290-1299$.

76. Vaithiyalingam S.R., Sastry S.V., Dehon R.H., Reddy I.K., Khan M.A., Long-term stability characterization of a controlled release gastrointestinal therapeutic system coated with a cellulose acetate pseudolatex, Pharmazie. 56, 66-69. 2001.

77. Sastry S.V., Reddy I.K., Khan M.A., Atenolol gastrointestinal therapeutic system:optimization of formulation variables using response surface methodology, 1997.J. Control. Release 45, 121-130. 
78. Sastry S.V., Khan M.A., Aqueous based polymeric dispersion: Plackett-Burman design for screening of formulation variables of atenolol gastrointestinal therapeutic system, 1998.Pharm. Acta Helv. 73 105-112.

79. Sastry S.V., Khan M.A, Aqueous-based polymeric dispersion: face-centered cubic design for the development of Atenolol gastrointestinal therapeutic system, 1998.Pharm. Dev. Technol. 3, 423-432.

80. Patil P.B., Gawali V.U., Patil H.N., Hardikar S.R., Bhosale A.V., Preparation and evaluation of mucoadhesive microspheres of atenolol and propranolol, 2009.Int.J.Pharm. Technol. Res. 1, 639-643.

81. B. Singh, S.K. Chakkal, N. Ahuja, Formulation and optimization of controlled release mucoadhesive tablets of atenolol using response surface methodology, 2006, AAPS PharmSciTech 7, Article 3 (http://www.aapspharmscitech.org).

82. Kim J., Shin S.C., Controlled release of atenolol from the ethylenevinyl acetate matrix, 2004. Int. J. Pharm. 273, 23-27.

83. Kenawy E.R., Mohamed H.E., Salem S.H., Controlled release of atenolol from freeze/thawed poly(vinyl alcohol) hydrogel, 2010. J. Saudi Chem. Soc. 14, 237-240.

84. Streubel A., Siepmann J., Bodmeier R., Floating matrix tablets based on low density foam powder: effects of formulation and processing parameters on drug release, 2003. Eur. J. Pharm. Sci. 18, 37-45.

85. Rouge N., Allemann E., Gex-Fabry M., Balant L., Cole E.T., Buri P., Doelker E., Comparative pharmacokinetic study of a floating multiple-unit capsule, a high- density multiple-unit capsule and an immediate-release tablet containing $25 \mathrm{mg}$ atenolol, 1998, Pharm. Acta Helv. 73, 81-87.

86. Reeves P.R., Barnfield D.J., Longshaw S., et al. Disposition and metabolism of atenolol in animals, 1978. Xenobiotica 8,(30)5-11.

87. Reeves PR, McAinsh J, McIntosh DAD, Winrow MJ. Metabolism of atenolol in man Xenobiotica 1978;8:313-20.

88. Brown H.C., Carruthers M.D., Johnston G.D., et al. Clinical pharmacologic observations on atenolol, a $\beta$-adrenoreceptor blocker, 1976. Clin Pharmac Ther. 20,524-34.

89. de Assis S.A., Ferreira B.S., Fernandes P., Guaglianoni D.G., Cabral J.M.S., Oliveira M.F., Gelatin-immobilized pectinmethylesterase for production of low methoxyl pectin, 2004. Food Chem. 86, 333-337.

90. Todorovic J., Ecke H., Lagerkvis A., Solidification with water as a treatment method for air pollution control residues, 2003. Waste Manage. 23, 621-629. 
91. Forte M.M.C., Cunha F.O.V., Santos J.H.Z., Zacca J.J., Ethylene and 1-butene copolymerization catalyzed by a Ziegler-Natta/metallocene hybrid catalyst through a 23 factorial experimental design, 2003. Polymer. 44, 1377-1384.

92. Bajdik J., Baki G., Kelemen A., Pintye-Hdi K., The effect of wetting of a powder mixture on the preparation of hydrophilic matrix granules with a high-shear granulator, 2008. Chem Eng. Res. Des. 86, 1-7.

93. Gohel M.C., Patel M.M., Amin A.F., Development of modified release diltiazem HCl tablets using composite index to identify optimal formulation, 2003. Drug Dev. Ind. Pharm. 29, 565-574.

94. Sertsou G., Butler J., Scott A., Hempenstall J., Rades T., Factors affecting incorporation of drug into solid solution with HPMC during solvent change co- precipitation, 2002. Int. J. Pharm. 245, 99-108.

95. Khanvilkar K.H., Ye Huang., Moore A.D., Influence of hydroxypropyl methylcellulose mixture, apparent viscosity, and tablet hardness on drug release using a $2^{3}$ full factorial design, 2002. Drug Dev. Ind. Pharm. 28, 601-608.

96. Davies B., Ellis S., Process analytical technology: the route to process understanding and control, 2005. Pharma. Technol. Eur. 17, 17-23.

97. Nürnberg E., Surmann P., Hagers Handbuch der pharmazeutischen Praxis, 5th. Ed. Bd. 2 Methoden. Springer Verlag, Berlin, New York. p. 60, 1991.

98. Pires de Aberu, L.R., Calafatti de Castro S.A., and Pedrazzoli J., Atenolol Quantification in Human Plasma by High- Performance Liquid Chromatography: Application to Bioequivalence Study, 2003. AAPS PharmSci5(2), 116-122.

99. Davies P.N., Worthington H.E.C., Podczeck F., Newton J.M., The determination of the mechanical strength of tablets of different shapes, 2007. Eur. J. Pharm. Biopharm. 67, 268-276.

100. van Santen E., Barends D.M., Frijlink H.W., Breaking of scored tablets: a review, 2002. Eur. J. Pharm. Biopharm., 53,139-145. 


\section{ACKNOWLEDGEMENT}

First of all I wish to express my sincere thanks to my supervisor Prof. Dr. Klára Pintye-Hódi for her support. I greatly appreciate her continuous help during the preparation of my thesis and providing the excellent facilities for this study. My warm gratitude to her for her patience, criticism, encouragement and numerous discussion during my Ph.D. work.

My warmest thanks to Prof. Dr. Piroska Szabó-Révész Head of the Department of pharmaceutical Technology, for providing me with the opportunity acceptance for Ph.D. in her Department.

My warmest thank to my co-supervisor Dr. János Bajdik for his good advice and help.

I express my kindest thanks to all my co-authors for their collaboration in this work. My warmest thank to all members of the department for their help and friendship.

My sincere thanks to my family and my friends for their support, encouragement and understanding attitude during these years. 
APPENDIX 
I. 


\title{
Effects of the wetting liquid and ethylcellulose on the properties of atenolol-containing pellets
}

\author{
E.I. Hamedelniel, J. Bajdik, T. Sovány, P. Kása Jr., K. Pintye-Hódi* \\ Department of Pharmaceutical Technology, University of Szeged, Eötvös u. 6, 6720 Szeged, Hungary \\ *Correspondence: klara.hodi@pharm.u-szeged.hu
}

\begin{abstract}
The aim of this study was to investigate the influence of the wetting liquid on the properties of pellets prepared by extrusion and spheronization. Different grades of ethylcellulose were used as matrix former, with microcrystalline cellulose as filler and atenolol as model active agent. Each ethylcellulose type was applied with water alone, or with a combination of water and ethanol. The formation of the matrix and the interaction of the components were evaluated via mechanical, dissolution and morphological studies on the pellets. The presence of ethanol in the wetting liquid led to a decrease in the liberation of the active agent in the first phase of the dissolution process, but also caused a reduction in the breaking hardness of the pellets. High viscosity grade ethylcellulose gave rise to a more relevant decrease in breaking hardness. The plasticity of the mass was influenced by variation of the wetting liquid.
\end{abstract}

Key words: Polymer-matrix composites - Deformation - Hardness testing - Scanning electron microscopy - Extrusion.

Pellets for pharmaceutical applications are defined as spherical, free-flowing granules with a narrow size distribution, typically varying in diameter between 500 and $1500 \mu \mathrm{m}$. Pellets are a popular multiparticulate pharmaceutical dosage form that are utilized for both immediate release and a number of different controlled or special release applications [1-3].

Different methods are used to formulate pellets. Extrusion followed by spheronization is currently a very popular and widely evaluated procedure. During the wetting/kneading stage, the addition of a fluid, characterized by its binding properties, ensures particle agglomeration and imparts the properties of flow, cohesion and deformability that are necessary for shaping. The extrusion and spheronization stages are mechanical processes that shape the wet heterogeneous medium, thereby modifying the organization of the different phases. This technique has been comprehensively reviewed [4]. It is very important to bear in mind that not all moistened powder mixtures can be successfully extruded and spheronized [5-7].

Newton [8] defined the specific requirements for a wetted mass to be suitable for extrusion and spheronization. For a successful process [9], microcrystalline cellulose is incorporated in most formulations, since it provides the wetted mass with the appropriate rheological properties [8].

Microcrystalline cellulose may be regarded as the standard as a structure-forming material; it has good binding properties that provide cohesiveness to a wetted mass, in this way aiding extrusion-spheronization [10-14]. Furthermore, in consequence of its large surface area and high internal porosity, it is able to absorb and retain a large quantity of water [15], thereby facilitating extrusion, improving the wetted mass plasticity and enhancing spheronization. Moreover, control of the movement of water through the plastic mass prevents phase separation during extrusion or spheronization [16].

Various pharmaceutical excipients can be used to modify the release of an active agent from pellets formulated by extrusion and spheronization. These components form a matrix system, which ensures appropriate liberation. Different types of polymers can be used to form soluble or insoluble systems. Their properties and the interactions between the components influence the dissolution of the active pharmaceutical ingredient. Ethylcellulose is a polymer highly suitable for film coating [17-19] and a variety of types of ethylcel- lulose are nowadays available as matrix formers [20, 21]. Ethylcellulose has been widely used in oral pharmaceutical formulations for various purposes, including moisture protection, taste masking [22] and controlled release. It is non-toxic, non-allergenic and non-irritant and has good film-forming properties [23].

Atenolol is a potent cardioselective $\beta$-adrenoreceptor blocking agent used for the treatment of hypertension, including hypertension in pregnancy [24]. The absorption of the drug following oral administration in humans and most laboratory animal species is rapid, but incomplete (50-60\%). It has been shown that atenolol is well absorbed at $\mathrm{pH}>7.5$ [25]. The normal $\mathrm{pH}$ of the human ileum is 7.2-7.6, and thus total absorption is impossible.

The aim of our present project was the formulation of a multiparticulate system containing atenolol with a view to increasing its bioavailability.Pellets which undergo a linear, but not too slow dissolution ( $80 \%$ in the first hour) and with appropriate mechanical properties (because of the subsequent enteric soluble coating) are necessary for this purpose. Ethanol and water were used in various combinations as wetting liquid and their effects on the properties of the pellets formed (breaking hardness and dissolution), on the formation of the matrix and on the interactions of the components were investigated.

\section{MATERIALS AND METHODS \\ 1. Materials}

Atenolol (Ariane Organochem Private Ltd., Mumbai, India) as model drug, and ethylcellulose (Ethocel standard 4,10 and 45 premium, Colorcon Ltd. Dartford, United Kingdom) and microcrystalline cellulose (Vivapur 103, Rettenmaier\&Söhne GmbH, Rosenberg, Germany) as pharmaceutical excipients were used. Ethanol $96 \%$ (Spectrum-3D, Debrecen, Hungary) and water were applied as wetting components.

\section{Preparation of pellets}

One hundred and fifty grams of powder mixture was prepared from $90 \mathrm{~g}$ of atenolol, $30 \mathrm{~g}$ of microcrystalline cellulose and $30 \mathrm{~g}$ of one or other of the different ethylcelluloses (Table I). To obtain a uniform mixture, the powder was blended at $50 \mathrm{rpm}$ for $10 \mathrm{~min}$ with a Turbula mixer (W.A. Bachofen, Basel, Switzerland).

Samples were prepared in a high-shear granulator (ProCepT 4M8 granulator, ProCepT nv, Zelzate, Belgium) with $95 \mathrm{~mL}$ of granulating 
Table I - Sample compositions.

\begin{tabular}{|c|c|c|c|c|c|c|c|c|c|}
\hline & & \multicolumn{3}{|c|}{ Powder } & \multicolumn{4}{|c|}{ Binder liquid } & \multirow{3}{*}{$\begin{array}{l}\text { Total liquid } \\
\text { amount } \\
\text { (mL) }\end{array}$} \\
\hline & & \multirow{2}{*}{ At $(\%)$} & \multirow{2}{*}{ MCC (\%) } & \multirow{2}{*}{ EC (\%) } & \multicolumn{2}{|c|}{ Water/mL } & \multicolumn{2}{|c|}{ Ethanol/mL } & \\
\hline & & & & & Pre & Post & Pre & Post & \\
\hline S1 & $\begin{array}{l}\text { A } \\
\text { B } \\
\text { C }\end{array}$ & $\begin{array}{l}60 \\
60 \\
60\end{array}$ & $\begin{array}{l}20 \\
20 \\
20\end{array}$ & $\begin{array}{c}20 \text { (EC4) } \\
20 \text { (EC10) } \\
20 \text { (EC45) }\end{array}$ & $\begin{array}{l}95 \\
95 \\
95\end{array}$ & & - & - & $\begin{array}{l}95 \\
95 \\
95\end{array}$ \\
\hline S2 & $\begin{array}{l}\text { A } \\
\text { B } \\
\text { C }\end{array}$ & $\begin{array}{l}60 \\
60 \\
60\end{array}$ & $\begin{array}{l}20 \\
20 \\
20\end{array}$ & $\begin{array}{c}20 \text { (EC4) } \\
20 \text { (EC10) } \\
20 \text { (EC45) }\end{array}$ & & $\begin{array}{l}80 \\
80 \\
80\end{array}$ & $\begin{array}{l}15 \\
15 \\
15\end{array}$ & & $\begin{array}{l}95 \\
95 \\
95\end{array}$ \\
\hline S3 & $\begin{array}{l}\text { A } \\
\text { B } \\
\text { C }\end{array}$ & $\begin{array}{l}60 \\
60 \\
60\end{array}$ & $\begin{array}{l}20 \\
20 \\
20\end{array}$ & $\begin{array}{c}20 \text { (EC4) } \\
20 \text { (EC10) } \\
20 \text { (EC45) }\end{array}$ & $\begin{array}{l}80 \\
80 \\
80\end{array}$ & & & $\begin{array}{l}15 \\
15 \\
15\end{array}$ & $\begin{array}{l}95 \\
95 \\
95\end{array}$ \\
\hline S4 & $\begin{array}{l}\text { A } \\
B \\
\text { C }\end{array}$ & $\begin{array}{l}60 \\
60 \\
60\end{array}$ & $\begin{array}{l}20 \\
20 \\
20\end{array}$ & $\begin{array}{c}20 \text { (EC4) } \\
20 \text { (EC10) } \\
20 \text { (EC45) }\end{array}$ & \multicolumn{4}{|c|}{ Mixture of $80 \mathrm{~mL}$ water $+15 \mathrm{~mL}$ ethanol } & $\begin{array}{l}95 \\
95 \\
95\end{array}$ \\
\hline
\end{tabular}

liquid i.e. either $95 \mathrm{~mL}$ of water alone, or a combination of water and ethanol ( $80 \mathrm{~mL} / 15 \mathrm{~mL}$, respectively): $15 \mathrm{~mL}$ of ethanol was added before or after the addition of $80 \mathrm{~mL}$ of water, or the two were added already mixed together. The amount of liquid employed was determined in preliminary studies.

The kneading parameters were as follows:

- impeller speed: $1500 \mathrm{rpm}$,

- chopper speed: $2000 \mathrm{rpm}$,

- dosing speed: $5 \mathrm{~mL} / \mathrm{min}$.

The wet mass obtained was extruded by a mini screw extruder (Caleva Ltd. Sturminster Newton, Dorset, United Kingdom) equipped with an axial screen with dies $1 \mathrm{~mm}$ in diameter and $4 \mathrm{~mm}$ in length, operating at $90 \mathrm{rpm}$. The jacked barrel of the extruder was cooled by water at $25 \pm 2{ }^{\circ} \mathrm{C}$. Each extrudate was collected in a container before it was spheronized. About $40 \mathrm{~g}$ of extrudate was spheronized at a time, on a $12 \mathrm{~cm}$ diameter spheronizer (Model-120, G.B. Caleva Ltd. Sturminster Newton, Dorset, United Kingdom) fitted with a cross-hatch grooved plate, for $3 \mathrm{~min}$ at $750 \mathrm{rpm}$. The pellets were dried under the same conditions at $40 \pm 2{ }^{\circ} \mathrm{C}$ for $24 \mathrm{~h}$.

\section{Evaluation of liquid uptake}

The Enslin number is a simple semiquantitative measure of the liquid (here water and ethanol) uptake of a powder and is equal to the amount of fluid absorbed by $1 \mathrm{~g}$ of powder $(\mathrm{mL} / \mathrm{g})$. An Enslin apparatus with a glass filter and a pipette with $0.01 \mathrm{~mL}$ accuracy were used for these experiments [26]. A monolayer of particles took up the maximum quantity of liquid possible through a filter paper under these conditions. $0.5 \mathrm{~g}$ of each powder was tested; three parallel experiments were performed.

\section{Dissolution test}

Pellets $(100 \mathrm{mg})$ were filled into gelatin capsules, which were placed into the basket of a dissolution tester (Erweka DT 700, Heusenstamm, Germany). The dissolution medium consisted of $900 \mathrm{~mL}$ of phosphate buffer ( $\mathrm{pH} 6.8) \mathrm{kept}$ at $37.0 \pm 0.5^{\circ} \mathrm{C}$. The rotational speed of the baskets was set at $100 \mathrm{rpm}$. The dissolution system was combined with an automatic sampling station. Samples of $5 \mathrm{~mL}$ were withdrawn at 10, 20, 30, 40, 50 and $60 \mathrm{~min}$. Atenolol was measured spectrophotometrically (Unicam He $\lambda$ ios Alpha, Spectronic Unicam, Cambridge, United Kingdom) at $\lambda_{\max }=224 \mathrm{~nm}$.

\section{Mechanical properties of the pellets}

The breaking strength of pellets was tested. The strength tester and the software were developed in our institute. The tester contains a special specimen holder and a jowl, and is connected to a computer via an interface. The loading indicates some stress in the sample and it can deform. As the surface area of the jowl is constant, the stressdeformation and force-time profiles are the same. Thus, not only can the ultimate deformation force be measured, but the process can be followed. The specimen is located horizontally on a special plate and the jowl moves vertically. Twenty parallel measurements were performed.

The measurement range was $0-200 \mathrm{~N}$, the speed of the stamp was $20 \mathrm{~mm} / \mathrm{min}$, and the output was $0-5 \mathrm{~V}$. The sensor was a Unicell force-measuring instrument, calibrated with the C9B $200 \mathrm{~N}$ cell.

\section{Morphological study}

The surfaces of various samples (S1A, S1C, S2A and S2C) before and after dissolution were tested with a scanning electron microscope (Hitachi S4700, Hitachi Scientific Instruments Ltd., Tokyo, Japan). A sputter coating unit (Polaron E5100, VG Microtech, United Kingdom) was used to charge the surfaces for the scanning electron microscopy measurements. The air pressure during the analyses was 1.3-13 mPa.

\section{RESULTS AND DISCUSSION \\ 1. Preformulation studies}

The uptake of liquids applied during pelletization is a very important parameter for the preparation of beads. The highest liquid uptake for both water and ethanol was detected for microcrystalline cellulose and the lowest for Ethocel4 (Table II). Microcrystalline cellulose has been described as a "molecular sponge" [16]. The microcrystalline cellulose particles are able to absorb and retain water in a manner similar to a sponge. The uptake of the mixture of water and ethanol applied was very similar to the water uptake for microcrystalline

Table II - Liquid uptakes of different samples, as Enslin numbers (mL/g).

\begin{tabular}{|l|c|c|c|c|c|}
\hline \multirow{2}{*}{ Liquid } & \multicolumn{5}{|c|}{ Type of powder } \\
\cline { 2 - 6 } & EC4 & EC10 & EC45 & MCC & At \\
\hline Water & $0.05 \pm 0.01$ & $0.07 \pm 0.02$ & $0.15 \pm 0.01$ & $2.76 \pm 0.04$ & $0.07 \pm 0.02$ \\
Ethanol & $0.25 \pm 0.02$ & $0.29 \pm 0.02$ & $0.61 \pm 0.01$ & $1.88 \pm 0.14$ & $1.09 \pm 0.05$ \\
Water + ethanol* $^{*}$ & $0.11 \pm 0.01$ & $0.23 \pm 0.01$ & $0.41 \pm 0.01$ & $2.71 \pm 0.02$ & $0.64 \pm 0.14$ \\
\hline
\end{tabular}

* $80 \mathrm{~mL}$ water $+15 \mathrm{~mL}$ ethanol. 
cellulose. The uptake of ethanol by atenolol was higher than that of water. As concerns the different grades of ethylcellulose, it is clear that, independently of the composition of the liquid, Ethocel 45 took up the highest amount of liquid. A gel-like layer formed around the particles in the ethanol. The viscosity of this mucous layer is known to depend on the type of ethylcellulose (Colorcon brochure).

\section{Dissolution}

The drug release from the pellets was studied with different dissolution kinetic models (first-order, Higuchi, Hopfenberg, RRSBW and Langenbucher). The results showed that the dissolution profile of the samples could be fitted best $\left(\mathrm{R}^{2}>0.95\right)$ with first-order kinetics (Equation 1), as is expected for the dissolution of water-soluble drugs from porous matrices.

$$
M_{t}=M_{0}\left(1-\mathrm{e}^{-k t}\right)
$$

where $M$ is the amount of API released from the preparation in time $t, M_{0}$ is the total amount of the drug, and $k$ is the dissolution rate of the process. However, even this model was unable to handle the presence of lag time, which can be observed especially in the case of samples prepared with ethanol. The dissolution was fitted with the Chapman-Richards growth model (Equation 2), which contains a shape parameter of the sigmoid-shaped curve (Figures 1 to 3), and can describe the lag time:

$$
M_{t}=M_{0}\left(1-\mathrm{e}^{-k t}\right)^{c}
$$

where $M_{t}$ is the amount of API released from the preparation in time $t, M_{0}$ is the total amount of the drug, $k$ is the dissolution rate of the process, and $c$ is the shape parameter of the curve, which refers to the observed lag time of the dissolution. The dissolution rates and shape parameters are displayed in Table III.

The results were analyzed with a two-way ANOVA model. The results showed significant differences in the rates of dissolution of the samples. On the basis of these statistical differences, the samples can be divided into three groups. The highest dissolution rates were observed for samples S2B and S3C, those for samples S3B and S4C were one-tenth less, and the other samples displayed even lower dissolution rate constants (Table III). A similar tendency was seen in the shape of the dissolution curves. There was a considerable lag time for samples with higher dissolution rates. The lag time was significantly shorter (Figure $4 b$ ) for samples prepared with water than for samples prepared with water and ethanol with the exception of sample S2C.A possible explanation of the manifestation of the lag time, therefore, is that the ethanol dissolves ethylcellulose, and during the drying period an ethylcellulose film is formed around the drug particles, this layer

Table III - Dissolution rate constants and correlation coefficients of dissolution curves.

\begin{tabular}{|c|c|c|c|c|}
\hline \multicolumn{2}{|c|}{ Sample } & $R^{2}$ values & $\begin{array}{c}\text { Dissolution rate } \\
\text { constants }\end{array}$ & $\begin{array}{c}\text { Shape } \\
\text { parameter }\end{array}$ \\
\hline S1 & A & 0.9968 & 0.04 & 1.35 \\
& B & 0.9919 & 0.03 & 1.40 \\
& C & 0.9956 & 0.05 & 1.70 \\
\hline S2 & A & 0.9951 & 0.04 & 1.88 \\
& B & 0.9928 & 0.07 & 3.73 \\
& C & 0.9870 & 0.04 & 1.66 \\
\hline S3 & A & 0.9871 & 0.04 & 2.32 \\
& B & 0.9875 & 0.05 & 2.66 \\
& C & 0.9929 & 0.07 & 3.74 \\
\hline S4 & A & 0.9903 & 0.03 & 2.16 \\
& B & 0.9968 & 0.04 & 3.10 \\
& C & 0.9895 & 0.05 & 2.09 \\
\hline
\end{tabular}

delaying the dissolution. Nevertheless, the mechanism and degree of film formation differed for the different grades of ethylcellulose. These differences are well revealed by the shifts in the maximal dissolution rates (Figure $4 a$ ) and lag times (Figure $4 b$ ). A possible explanation of this phenomenon is that the quality of the films is sensitive to the solvent mixture applied, depending on the chain-length of the ethylcellulose. In the case of short chain-length ethylcelluloses (Ethocel4), the films formed are rigid and break easily, and are not able to influence the drug dissolution. The quality of the films improves significantly

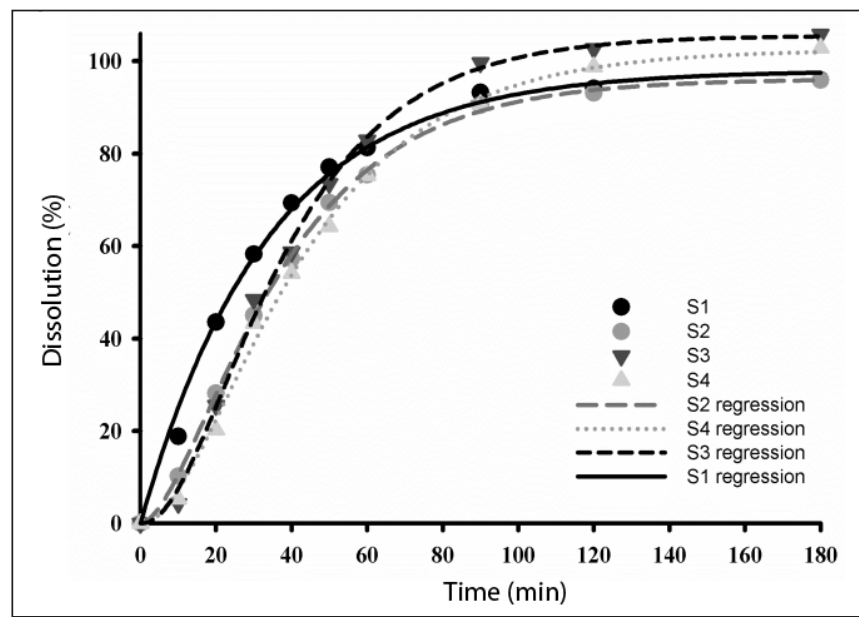

Figure 1 - Dissolution of Atenolol from pellets containing EC4.

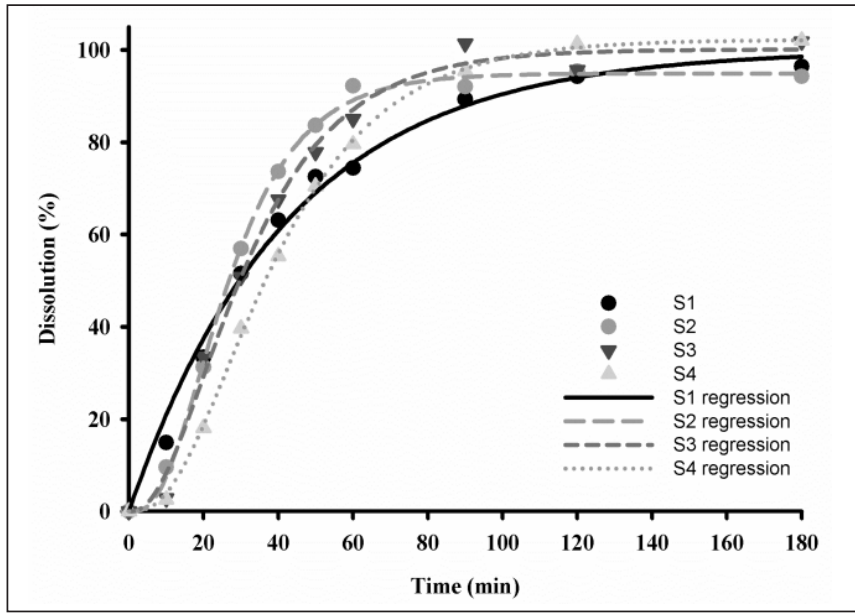

Figure 2 - Dissolution of Atenolol from pellets containing EC10.

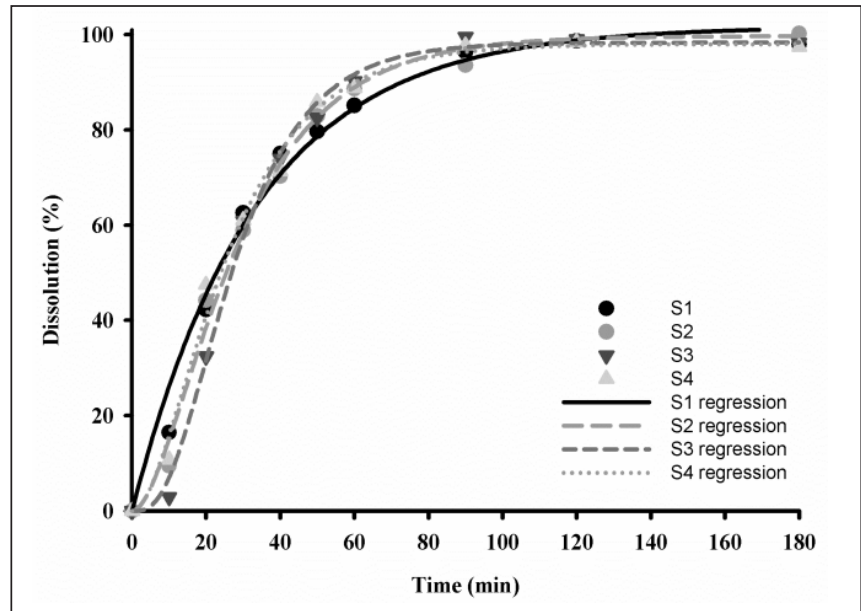

Figure 3 - Dissolution of Atenolol from pellets containing EC45. 


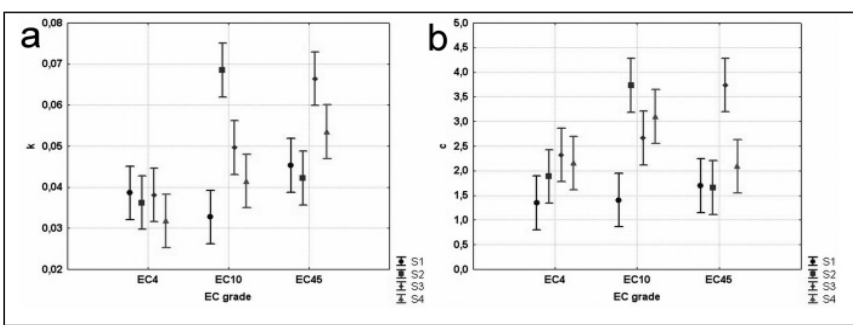

Figure 4 - Box-plots of the statistical analysis: effect of the EC grade and the method of preparation for the dissolution rate constants (a) and lag-time (b).

with the lengthening of the polymer chains, and the film formation can then modify the rate of dissolution of the drug. On the basis of these results, the samples prepared with water were chosen for further investigation.

\section{Mechanical properties of the pellets}

The evaluation of the pellet deformation process involved not only determining the breaking hardness, but also studying the deformation curve. The shapes of the breaking curves of the pellets were very similar for the samples prepared with water (Figure 5). They mainly comprised three phases: The first section (Figure $5 a, 1$ ) is indicative of elastic deformation. The pellet behaves as a Kelvin body, in which the Hooke component dominates. The relationship between the loading and the stress can be written as follows:

$$
\overline{\mathrm{t}}=\mathrm{E}(\overline{\mathrm{d}}-\bar{\delta})
$$

where $\overline{\mathrm{t}}$ is the Cauchy stress tensor, $\mathrm{E}$ the elasticity modulus, $\overline{\mathrm{d}}$ the tensor of deformation and $\bar{\delta}$ the unit tensor.

In the second section (Figure $5 a, 2$ ), the pellet behaves as a SaintVenant body, exhibiting a permanent deformation after reaching the breaking stress (plastoelastic section), which leads to breaking of the crystal structure.

Third section (Figure 5a,3): during the total deformation process, the behavior of the binder film as a Hooke body predominates until the total breaking of the pellet.

The samples containing ethanol gave curves with more peaks. This phenomenon revealed that the internal structure of these samples was inhomogeneous.

A probable liquid-independent factor determining the pellet structure could be the plastic deformation of the particles containing ethylcellulose during the extrusion. However, other liquid-dependent structure-determining components cause changes in the shape of the deformation curve.

For the samples prepared in water, the main binder in the pellet was the microcrystalline cellulose. It is known that, according to the "crystallite-gel model" [27], microcrystalline cellulose particles are broken down into smaller units and even partly into single crystals of colloidal size during granulation and extrusion in the presence of water. The resulting crystallites and porous particles form a coherent gel-like network (with a high fraction of an insoluble solid phase) and immobilize the liquid. Over a particular range of water content, which relates to acceptable gel strength, extrusion and spheronization become possible. This effect improves the plasticity of the wetted mass and enhances the spheronization, and is therefore a pellet structure-forming parameter which can determine the mechanical properties. In this case, other possible structure-forming phenomena could include the recrystallization of atenolol and the partial melting of ethylcellulose during the extrusion. The presence of ethanol in the wetting process significantly influenced the gel formation and solubility of atenolol. The film-forming mucous solution of ethylcellulose was capable of changing the structure of the "microcrystalline cellulose gel". The

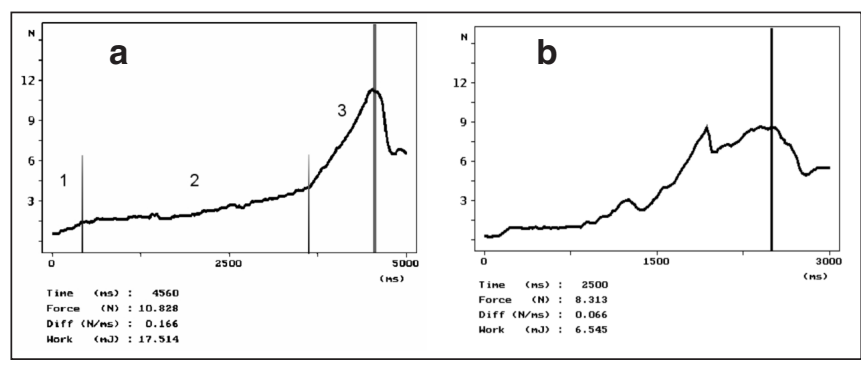

Figure 5-Deformation curves of pellets containing EC45 (a:S1C, b:S3C).

viscosity of this ethanolic solution exerted a disturbing effect. The inhomogeneity in the "microcrystalline cellulose gel-like" matrix and the film formation of ethylcellulose had significant effects on the properties of the pellets. A lower breaking hardness of samples prepared with ethanolic solution could be detected in the case of ethylcelluloses with higher viscosity grades (Table IV). The difference appeared to be significant in the two-way ANOVA test. There was no significant effect of the wetting liquid on the mechanical properties of the pellets prepared with Ethocel4. This can be explained by the low viscosity of its ethanolic solution. Its effect on the plasticity of the mass was therefore negligible. In the case of water-containing samples there was not a significant decreasing tendency with increasing water uptake of the ethylcellulose. The effect of the hydrated ethylcellulose therefore increased, and accordingly the breaking hardness for the S1C decreased. For each liquid, the lowest breaking force was detected for the sample containing Ethocel45. The samples prepared with this polymer exhibited decreases in breaking force in the sequence water $>$ aqueous ethanol solution. The extent of the alteration was similar in every case.

A reduction in breaking hardness was also detected for Ethocel10, but it exhibited a less considerable change for S4B. In this case, the aqueous ethanol did not give rise to a more dramatic change in the "crystallite-gel model" of microcrystalline cellulose. The liquid uptake of microcrystalline cellulose was very similar for water and aqueous ethanol, and the presence of an ethylcellulose gel can therefore disturb the formation of the particles. The hydration of Ethocel10 induced a lower extent of alteration than that for Ethocel45, the hydration of which is better in aqueous ethanol.

\section{Morphology of surface of pellets}

The surface morphology of the nearly spherical pellet before dissolution testing for samples S1A and S1C is presented in Figure 6. The surface of both was slightly rough; the microcrystalline cellulose fibers, small crystals of atenolol and some pieces of EC-film (Figure 6, $\mathrm{A}, \mathrm{B} \rightarrow$ ) could be detected.

Table IV - Breaking hardness of pellets.

\begin{tabular}{|c|c|c|}
\hline \multicolumn{2}{|c|}{ Sample } & Breaking force (N) \\
\hline S1 & A & $13.7 \pm 2.5$ \\
& B & $11.9 \pm 2.2$ \\
& C & $11.2 \pm 1.3$ \\
\hline S2 & A & $13.5 \pm 1.6$ \\
& B & $8.8 \pm 1.4$ \\
& C & $7.8 \pm 1.0$ \\
\hline S3 & A & $12.4 \pm 1.9$ \\
& B & $8.8 \pm 1.5$ \\
& C & $7.3 \pm 1.1$ \\
\hline S4 & A & $13.2 \pm 2.3$ \\
& B & $10.1 \pm 1.4$ \\
& C & $7.2 \pm 0.9$ \\
\hline
\end{tabular}




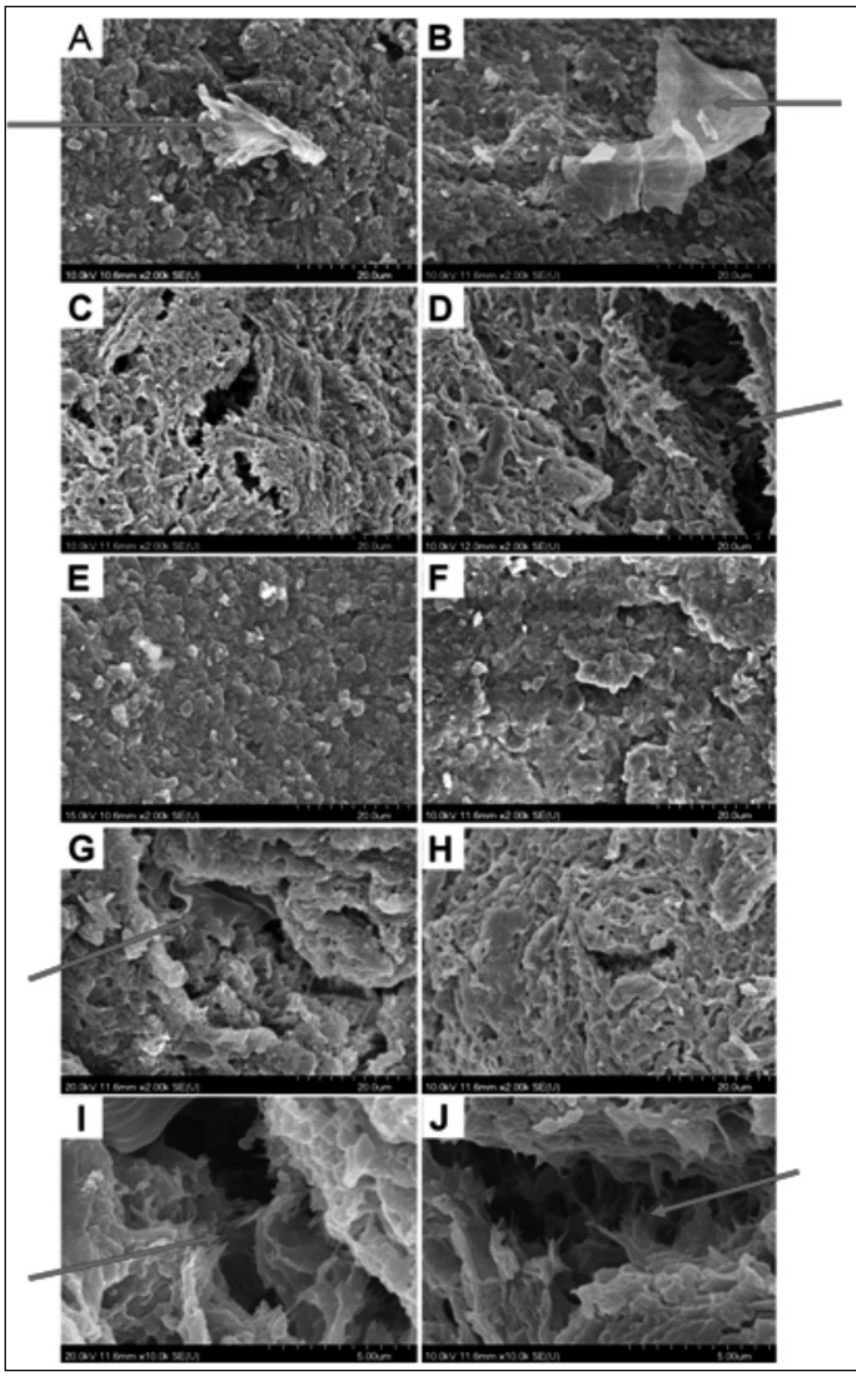

Figure 6 - Morphology of surface of pellets. A: S1A before dissolution. B: S1C before dissolution. C: S1A after dissolution. D: S1C after dissolution. E: S3A before dissolution. F: S3C before dissolution. G: S3A after dissolution. $\mathrm{H}$ : S1C after dissolution. I: S3A after dissolution. J: $\mathrm{S} 1 \mathrm{C}$ after dissolution.

On contact with the dissolution medium, the particles that are immediately wetted change phase and then diffuse through the outer boundary layer exposed to the medium. The liquid gradually penetrates the porous network and the dissolved molecules then have to make an additional diffusional displacement within the porous pellets [28]. Dissolution leads to an increase in the size of the wetted pores (Figure $6, \mathrm{D} \rightarrow$ ), and the disparity of the wet zones generates a porosity gradient between the core and the periphery of the pellet, leading to changes in the structure and shape of the pellet. In the deeper layer, a piece of the Et-film (Figure 6, G, I $\rightarrow$ ) or split film shreds can be seen (Figure $6, \mathrm{~J} \rightarrow$ ). After dissolution, the surface was very rough and exhibited numerous cracks and pores caused by the diffusion of atenolol in sample S1C rather than in sample S1A. Sample S1C displayed the formation of a film-like layer of microcrystalline cellulose and partial melting of the ethylcellulose at the temperature of production during extrusion. The jacket of the extruder was cooled, but the local increase of temperature was unavoidable.

In contrast, a smoother surface was observed on sample S3 than on sample S1. Microcrystalline cellulose and atenolol particles were also seen for the untreated samples. After dissolution, large cracks and pores were formed, due to the liberation of the active agent. The film formation on sample $\mathrm{S} 3$ was more significant than that on sample
S1; this may be due to the use of ethanol, in which ethylcellulose is soluble. When ethanol was used in the second step of the wetting, the possibility of film formation on the surface of the particles was higher than when ethanol was applied initially. Clearly, not only the selective appearance of the ethylcellulose film formed from the solution, but also the "microcrystalline cellulose film" and the film formed after hot melting must be considered. Because of the application of ethanol, the presence of the additional film explained the delayed dissolution of these samples. Its integrity (determined by the hardness) was therefore a very important rate-limiting parameter.

On the basis of this study, it can be concluded that water or different water/ethanol combinations were appropriate to formulate pellets from powder mixtures containing different ethylcelluloses, microcrystalline cellulose and atenolol. The dissolution profile of the samples prepared with water follows first-order kinetics. For the samples where the granulating liquid also contained ethanol, the dissolution changed, and a lag time was observable. In this case, the ethanol dissolves the ethylcellulose, and in the drying period an ethylcellulose film is formed around the drug particles, this layer delaying dissolution. The ethanol also caused a reduction in the breaking strength of pellets prepared from Ethocel45. Both the breaking force and the profile of the deformation curve changed. A lower degree of alteration was detected for Ethocel10, and the least for Ethocel4. There was a very good correlation between this tendency and the increase in the level of hydration of the ethylcellulose. This process caused a disturbance in the formation of the "crystallite-gel model," which is responsible for the plasticity of the mass and hence the properties of pellets prepared by extrusion and spheronization. The breaking hardness of the pellets containing high-viscosity grade ethylcellulose was therefore the lowest, and this was particularly sensitive to the wetting liquid. Finally, it may be stated that the application of ethanol in the wetting liquid led to a decrease in the dissolution in the first phase, but also caused a reduction in the breaking strength of the pellets. The formation and the structure of the pellets differed for the sample prepared with the liquid containing ethanol.

\section{REFERENCES}

1. Umprayn K., Chitropas P., Amarekajorn S. - Influence of process variables on physical properties of the pellets using an extruder and spheronizer. - Drug Dev. Ind. Pharm., 25, 45-61 1999.

2. Law M.F., Deasy P.B. - Use of hydrophilic polymers with microcrystalline cellulose to improve extrusion-spheronization. - Eur. J. Pharm. Biopharm., 45, 57-65, 1998.

3. Vervaet C., Baert L., Remon J.P. - Extrusion-spheronization: literature review. - Int. J. Pharm., 116, 131-146, 1995.

4. Trivedi N.R., Rajan M.G., Johnson J.R., Shukla A.J. - Pharmaceutical approaches to preparing pelletized dosage forms using the extrusion-spheronisation process. - Critical Rev. Ther. Drug Carrier Syst., 24, 1-40, 2007.

5. Agrawal A.M., Howard M.A., Neau S.H. - Extruded and spheronized beads containing no microcrystalline cellulose: influence of formulation and process variables. - Pharm. Dev. Technol., 9, 197-217, 2004.

6. Tho I., Sande S.A., Kleinebudde P. - Pectinic acid, a novel excipient for production of pellets by extrusion/spheronization: preliminary studies. - Eur. J. Pharm. Biopharm., 54, 95-99, 2002.

7. Basit A.W., Newton M., Lacey L.F. - Formulation of ranitidine pellets by extrusion-spheronization with little or no microcrystalline cellulose. - Pharm. Dev. Technol., 4, 499-505, 1999.

8. Newton J.M. - Extrusion and extruders. - In: Swarbrick J., Boylan J.C.(Eds.), Encyclopedia of Pharmaceutical Technology, Marcel Dekker Inc., New York and Basel, 2002, pp. 1220-1236.

9. Shah R.D., Kabadi M., Pope D.G., Augsburger L.L. - Physico- 
mechanical characterization of the extrusion-spheronization process. 2. Rheological determinants for successful extrusion and spheronization. - Pharm. Res., 12, 496-507, 1995.

10. Berggren J., Alderborn G. - Effect of drying rate on porosity and tableting behaviour of cellulose pellet. - Int. J. Pharm., 227, 81-96, 2001.

11. Habib Y.S., Shangraw R.F. - Effect of different drying techniques on the physico-mechanical properties of beads containing microcrystalline cellulose produced by extrusion and spheronization. - Pharm. Res., 14, 5-14, 1997.

12. Dyer A.M., Khan K.A., Aulton M.E. - Effects of the drying method on mechanical and release properties of pellets prepared by extrusion-spheronization. - Drug Dev. Ind. Pharm., 20, 30453068, 1994.

13. Kleinebudde P. - Shrinking and swelling properties of pellets containing microcrystalline cellulose and low substituted hydroxy propyl cellulose. Part I. Shrinking properties. - Int. J. Pharm., 109, 209-219, 1994.

14. Bataille B., Ligarski K., Jacob M., Thomas C., Duru C. - Study of the influence of spheronization and drying conditions on the physico-chemical properties of neutral spheroids containing Avicel PH101 and lactose. - Drug Dev. Ind. Pharm., 19, 653-671, 1993.

15. Sonaglio D., Bataille B., Ortigosa C., Jacob M. - Factorial design in the feasibility of producing Microcel MC 101 pellets by extrusion/spheronization. - Int. J. Pharm., 115, 53-60, 1995.

16. Fielden K.E., Newton J.M., Rowe R.C. - The influence of lactose particle size on spheronization of extrudate processed by a ram extruder. - Int. J. Pharm., 81, 205-224, 1992.

17. Ye Z.-W., Rombout P., Remon J.P., Vervaet C., Van Den Mooter G. - Correlation between the permeability of metoprolol tartrate through plasticized isolated ethylcellulose/hydroxypropylmethylcellulose films and drug release from reservoir pellets. - Eur. J. Pharm. Biopharm., 67, 485-490, 2007.

18. Iyer E.M., Augsburger L.L., Parikh D.M. - Evaluation of drug layering and coating: effect of process mode and binder level. - Drug Dev. Ind. Pharm., 19, 981-998, 1993.

19. Wallace J.W. - Cellulose derivatives and natural products utilized in pharmaceutics. - In: Swarbrick J., Boylan J.C. (Eds.), Encyclopedia of Pharmaceutical Technology, Marcel Dekker Inc., New York and Basel, 2002, pp. 319-339.

20. Verhoeven E., De Beer T.R.M., Van den Mooter G., Remon J.P., Vervaet C.V. - Influence of formulation and process parameters on the relelase characteristics of ethylcellulose sustained-release mini-matrices. - Eur. J. Pharm. Biopharm., 69, 312-319, 2008.

21. Huang J., Wigent R.J., Bentzley C.M., Schwartz J.B. - Nifedipine solid dispersion in microparticles of ammonio methacrylate copolymer and ethylcellulose binary blend for controlled drug delivery: effect of drug loading on release kinetics. - Int. J. Pharm., 319, 44-54 2006.

22. DeMerlis C.C., Schoneker D.R., Borzelleca J.F. - A subchronic toxicity study in rats and genotoxicity tests with an aqueous ethylcellulose dispersion. - Food Chem. Toxicol., 43, 1355-1364, 2005.

23. Naelapää K., Veski P., Kristensen H.G., Bertelsen P. - Coating of potassium chloride with ethylcellulose aqueous dispersion, effects of processing parameters on film formation and film properties. - Eur. J. Pharm. Sci., 32, S20, 2007.

24. Nikam A.P., Bodhankar S.L., Piplani P., Bansal J., Thakurdesa A. - Beta-adrenoreceptor blocking and antihypertensive activity of pp-24 a newly synthesized aryloxypropanolamie derivative. - J. Pharm. Pharmcol., 60, 1501-1506 2008.

25. Tabacova S.A., Kimmel C.A.-Atenolol:pharmacokinetic/dynamic aspects of comparative developmental toxicity.- Reprod. Toxicol., 16, 1-7, 2002.

26. Nürnberg E., Surmann P. - Hagers Handbuch der pharmazeutischen Praxis, Bd. 2 Methoden. - 5. Edition, Springer Verlag, Berlin, New York, 1991, p. 60.

27. Kleinebudde P. - The crystallite-gel-model for microcrystalline cellulose in wet-granulation, extrusion, and spheronization. Pharm. Res., 14, 804-809, 1997.

28. Costa P., Sousa-Lobo J.M. - Modelling and comparison of dissolution profiles. - Eur. J. Pharm. Sci., 13, 123-139, 2001.

\section{ACKNOWLEDGEMENTS}

The authors wish to express their gratitude to Colorcon for supplying ethylcellulose samples.

\section{MANUSCRIPT}

Received 1 october 2010, accepted for publication 20 January 2011. 


\section{II.}




\title{
Optimization of preparation of matrix pellets containing ethylcellulose
}

\author{
Elnazeer I. Hamedelniel, János Bajdik, Klára Pintye-Hódi* \\ Department of Pharmaceutical Technology, University of Szeged, Eötvös u. 6, H-6720 Szeged, Hungary
}

\section{A R T I C L E I N F O}

\section{Article history:}

Received 19 June 2009

Received in revised form 14 October 2009

Accepted 7 December 2009

Available online 16 December 2009

\section{Keywords:}

Breaking hardness

Dissolution

Ethylcellulose

Extrusion/spheronization

Factorial design

Wetting

\begin{abstract}
A B S T R A C T
The aim of this study was to investigate the effects of the parameters of the spheronization and the nature of the wetting liquid on the properties of matrix pellets prepared by extrusion and spheronization. Ethylcellulose was used as a matrix former, microcrystalline cellulose as a filler, atenolol as a model active agent, and water and a water-ethanol mixture as liquids. The formation of the pellets and the interactions of the components were evaluated via mechanical, dissolution and morphological studies on the pellets. A factorial design was used to determine the effects of the evaluated factors. It was concluded that significant effects were exerted not only by the operational parameters, but also by the nature of the liquid. The breaking hardness and the dissolution revealed that the ethanol in the liquid caused changes in the wettability of the components and consequently in the matrix structure. This was explained by a comparison of the relative importance of the factors. The alterations induced by ethanol were preferable in the dissolution, because the possibility of the burst effect in the first phase of dissolution can then be avoided. However, it is not favourable as concerns the sphericity and the mechanical properties of the pellets.
\end{abstract}

(c) 2009 Elsevier B.V. All rights reserved.

\section{Introduction}

In recent years there has been a great effort to develop controlled drug release systems via which to achieve the optimum therapeutic effect of drugs; the drug concentration is maintained in the therapeutic window for a period of time, thereby ensuring sustained therapeutic action. In several diseases (such as bronchial asthma, hypertension, rheumatic disease and myocardial infarction) and for the control of body functions (blood pressure, and the levels of many hormones, e.g. aldosterone, renin and cortisol) influenced by circadian rhythms, delayed or pulsatile drug release could be the optimum approach [1-4].

Pellets offer biopharmaceutical advantages in terms of more even and predictable distribution and transportation in the gastrointestinal tract, which is fairly independent of the nutritional state [5]. The interest in pellets as a dosage form (filled into hard gelatin capsules) has increased continuously, for their multiparticulate nature offers important pharmacological and technological advantages over conventional single-unit solid dosage forms [6]. Particle morphology is a key determinant of the behaviour of bulk solids and multiparticulate systems: many of the physical and chemical properties of such systems depend on the particle shape and surface geometry [7]. Thus, the morphological characterization of particles is of great importance in pharmaceutical technology.

\footnotetext{
* Corresponding author. Tel.: +36 62545576; fax: +36 62545571.

E-mail address: klara.hodi@pharm.u-szeged.hu (K. Pintye-Hódi).
}

In the field of granulation and pelletization, this characterization is critical for production steps such as capsule filling, and especially the coating of pellets. The morphological characterization of a particle basically requires three different aspects to be taken into account [8]. First, the particle projection must be assigned to that geometric shape category (e.g. a circle) which best describes it. Second, the morphological analysis should include an assessment of the roundness of the particle, and the sharpness/roundness of the vertices, edges and sides of the particle. Third, the surface texture must be evaluated.

The extrusion and spheronization stages are mechanical processes that shape the wet heterogeneous medium, thereby modifying the organization of the different phases. Drying finalizes the textural characteristics of the product by densifying the medium through mechanical shrinkage induced by extraction of the liquid phase. Not all moistened powder mixtures can be successfully extruded. Newton defined the specific requirements for a wetted mass to be suitable for extrusion and spheronization [9]. For successful extrusion and spheronization, microcrystalline cellulose (MCC) is incorporated in most formulations that are to be processed in this way [10]. MCC may be regarded as the standard as a structure-forming material: it has good binding properties that provide cohesiveness to a wetted mass, thereby aiding extrusion-spheronization [11-15]. Moreover, in consequence of its large surface area and high internal porosity, it is able to absorb and retain a large quantity of water [16]. Various pharmaceutical excipients can be used to modify the liberation of an active agent from the pellets formulated by extrusion and spheronization. 
These components form a matrix system, which ensures appropriate liberation. Different types of polymers can be used to form soluble or insoluble systems. Their properties and the interactions between the components influence the dissolution of the active pharmaceutical ingredient. Ethylcellulose (EC) is a polymer that is highly suitable for film coating [17-19]. It has been widely used in oral pharmaceutical formulations for various purposes, including moisture protection, taste masking and controlled release [20]. It is non-toxic, non-allergenic and non-irritant, and has good filmforming properties $[21,22]$. It is not soluble in water and a special pretreatment is therefore necessary for the application of aqueous systems [23]. Another possibility via which to process this agent is the application of ethanol as its solvent. The film formation in the solid polymer matrix significantly influences the texture of the dosage form and hence the liberation of the active agent.

Atenolol (At) is a cardioselective $\beta$-adrenoreceptor blocking agent used for the treatment of hypertension, including hypertension in pregnancy [24,25]. The absorption of the drug following oral administration in humans and most laboratory animal species is rapid, but incomplete (50-60\%). When given intravenously, At is almost completely excreted in the urine. Upon oral administration, between $40 \%$ and $50 \%$ of the unchanged compound is recovered in the urine and $50 \%$ is recovered unchanged in the faeces due to incomplete intestinal absorption in the human and in most experimental species, except for the dog. There is no evidence that there is an alternative biliary route for excretion in either humans or experimental animals [26,27]. At is well absorbed at $\mathrm{pH}>7.5$ [28].

The normal $\mathrm{pH}$ of the human ileum is 7.2-7.6, and thus total absorption is impossible. Co-administration of this active agent with an alkalizing component in a multiparticulate system in order to ensure an appropriately alkaline micromilieu is therefore reasonable. The good water solubility of At means that a polymer is required to control the parallel release of At and the alkalizing component.

The aim of our present project was the formulation of a multiparticulate system containing At with a view to increasing its bioavailability. Pellets without a burst effect which undergo linear, but not too slow dissolution (80\% in the first hour) and with appropriate mechanical properties (because of the subsequent enteric soluble coating) are necessary for this purpose. We set out to investigate the effects of the parameters of spheronization on the properties of pellets containing At, MCC and EC without alkalizing components, and to determine the main factors which can influence the preparation of such pellets. A $2^{3}$ full factorial design was utilized to optimize the circumstances applied during pelletization. Previously, the effects of the parameters of spheronization on the shape of the pellets and on the dissolution were tested [29-31]. However, the extensive testing of breaking hardness was not performed. In the present study, this parameter was also involved and a newly developed apparatus was used for this test. The effects of the nature of the wetting liquid applied for pelletization on the parameters of the final pellets were also examined.

\section{Materials and methods}

\subsection{Materials}

At (Ariane Organochem Private Ltd., Mumbai, India) was applied as model drug, with Ethocel standard 10 premium EC (Colorcon Ltd., Dartford, England) and MCC type 103 (Vivapur 103, Rettenmaier\&Söhne GmbH, Rosenberg, Germany) as pharmaceutical excipients. Ethanol 96\% (Spectrum-3D, Debrecen, Hungary) and water served as wetting components.

\subsection{Preparation of pellets}

$150 \mathrm{~g}$ of powder mixture was prepared from $75 \mathrm{~g}$ of At, $45 \mathrm{~g}$ of MCC and $30 \mathrm{~g}$ of EC 10 . To obtain a uniform mixture, the powder was blended at $50 \mathrm{rpm}$ for $10 \mathrm{~min}$ with a Turbula mixer (W.A. Bachofen, Basel, Switzerland).

Wetting was performed in a high-shear mixer (ProCepT 4M8 granulator, ProCepT nv, Zelzate, Belgium) with $110 \mathrm{ml}$ of granulating liquid, which was either $110 \mathrm{ml}$ of water alone, or a combination of water and ethanol $(92 \mathrm{ml} / 18 \mathrm{ml}$, respectively). The amount of ethanol used was based on the results of previous studies of the effects of the wetting liquid on the properties of EC-containing pellets prepared by extrusion and spheronization, in which different grades of ECs and water alone or mixed with ethanol were used.

The kneading parameters were as follows:

\section{Impeller speed $1500 \mathrm{rpm}$ \\ Chopper speed $2000 \mathrm{rpm}$ \\ Dosing speed $5 \mathrm{ml} / \mathrm{min}$}

The wet mass obtained was extruded by a mini screw (Caleva Ltd., Sturminster Newton, Dorset, UK) equipped with an axial screen with dies $1 \mathrm{~mm}$ in diameter and $4 \mathrm{~mm}$ in length, operating at $90 \mathrm{rpm}$. The jacked barrel of the extruder was cooled by water at $25 \pm 2{ }^{\circ} \mathrm{C}$. Each extrudate was collected in a container before it was spheronized. About $40 \mathrm{~g}$ of extrudate was spheronized at a time, on a spheronizer $12 \mathrm{~cm}$ in diameter (Model120, G.B. Caleva Ltd., Sturminster Newton, Dorset, UK) fitted with a cross-hatch grooved plate, for 3 and $10 \mathrm{~min}$ at 750 and $1000 \mathrm{rpm}$. The pellets were dried under the same conditions at $40 \pm 2{ }^{\circ} \mathrm{C}$ for $24 \mathrm{~h}$.

\subsection{Morphological study}

The particle size and the shape of the pellet surface were studied by using a system consisting of a stereomicroscope (Zeiss Stemi 2000-C, Carl Zeiss GmbH, Vienna, Austria) and a ring light with a cold light source (Leica KL 1500, Cambridge, UK). A Quantimet 500 (Q500MC) image processing and analysis system (Leica Cambridge Ltd., Cambridge, UK) was used. The aspect ratio was utilized for the evaluation of the shape of the particles.

\subsection{Mechanical properties of the pellets}

The breaking hardness was tested for pellets with diameters measuring between 1000 and $1200 \mu \mathrm{m}$. The strength tester and the software were developed in our institute. The tester contains a special specimen holder and a stamp, and is connected to a computer via an interface. Thus, not only can the ultimate deformation force be measured, but the process (force-time and force-displacement curves) can also be followed. The specimen is located horizontally on a special plate and the stamp moves vertically. If the measured plot (force-time) is parallel to the $x$ axis, the deformation is viscoelastic; if the plot rises linearly, the deformation is elastic. Twenty parallel measurements were performed.

The measurement range was $0-200 \mathrm{~N}$, the speed of the stamp was $20 \mathrm{~mm} / \mathrm{min}$, and the output was $0-5 \mathrm{~V}$. The sensor was a Unicell force measuring instrument, calibrated with the C9B $20 \mathrm{kN}$ cell.

Mechanical properties of pellets can be influenced by their residual moisture content. This was therefore determined with a moisture analyser (HR73 Halogen Moisture Analyser, MettlerToledo GmbH, Greifensee, Switzerland). A temperature of $105^{\circ} \mathrm{C}$ was applied until constant weight was attained. 
Table 1

Values of factors.

\begin{tabular}{lcc}
\hline Factor & Low level $(-)$ & High level $(+)$ \\
\hline Amount of ethanol $(X 1)$ & $0 \%$ & $16.3 \%$ \\
Speed of spheronization $(X 2)$ & $750 \mathrm{rpm}$ & $1000 \mathrm{rpm}$ \\
Duration of spheronization $(X 3)$ & $3 \mathrm{~min}$ & $10 \mathrm{~min}$ \\
\hline
\end{tabular}

Table 2

Designation of samples.

\begin{tabular}{lccc}
\hline Sample & $X 1(\%)$ & $X 2(\mathrm{rpm})$ & $X 3(\mathrm{~min})$ \\
\hline S1 & 16.3 & 750 & 10 \\
S2 & 16.3 & 750 & 3 \\
S3 & 16.3 & 1000 & 3 \\
S4 & 16.3 & 7000 & 10 \\
S5 & 0 & 750 & 10 \\
S6 & 0 & 1000 & 3 \\
S7 & 0 & 1000 & 3 \\
S8 & 0 & & 10
\end{tabular}

\subsection{Dissolution tests}

Pellets $(100 \mathrm{mg})$ were filled into gelatin capsules, which were placed into the basket of a dissolution tester (Erweka DT 700, Heusenstamm, Germany). The dissolution medium consisted of $900 \mathrm{ml}$ of phosphate buffer ( $\mathrm{pH} 6.8$ ) kept at $37.0 \pm 0.5^{\circ} \mathrm{C}$. The rotational speed of the baskets was set at $100 \mathrm{rpm}$. The dissolution system was combined with an automatic sampling station. Samples of $5 \mathrm{ml}$ were withdrawn at 10,20,30, 40, 50 and $60 \mathrm{~min}$. At was measured spectrophotometrically (Unicam Heגios Alpha, Spectronic Unicam, Cambridge, UK) at $\lambda_{\max }=224 \mathrm{~nm}$. Three parallel tests of dissolution were performed.

\subsection{Evaluation of liquid uptake of powder mixture}

The Enslin number is a simple semiquantitative measure of the liquid uptake of a powder and is equal to the amount of fluid absorbed by $1 \mathrm{~g}$ of powder $(\mathrm{ml} / \mathrm{g}$ ). An Enslin apparatus with a glass filter and a pipette with $0.01 \mathrm{ml}$ accuracy were used for these experiments [32]. A monolayer of particles took up the maximum quantity of liquid possible through a filter paper under these conditions. The uptake of the applied wetting liquid by $0.5 \mathrm{~g}$ of powder was determined; three parallel experiments were performed.

\subsection{Factorial design}

The factorial design is a method often used to accelerate the solution of problems. This method has been utilized in various branches of science and industry, e.g. food [33], environmental management [34], chemistry [35], and pharmaceutical technology [36-39]. The mathematically determined effects of different factors are compared by means of this technique, this information being very useful for the application of process analytical technology at the heart of which is the acquisition of a deep understanding of the manufacturing process [40].

A $2^{3}$ full factorial design was applied to choose the relevant factors (granulation liquid, water alone or with ethanol, spheronization speed and duration of spheronization) influencing three operational parameters: the dissolution, the breaking strength of the pellets and the shape of the pellets (sphericity)). The levels of the factors are to be found in Table 1 . The experiments were performed in randomized sequence. The samples are designated in Table 2 . The following approach, containing the interactions of the factors, was used to determine the response surface and the relative effects of the factors $(b)$ :

$y=b_{0}+b_{1} X_{1}+b_{2} X_{2}+b_{3} X_{3}+b_{12} x_{1} x_{2}+b_{13} x_{1} x_{3}+b_{23} x_{2} x_{3}$
Table 3

Liquid uptakes of different samples, as Enslin numbers ( $\mathrm{ml} / \mathrm{g}$ ).

\begin{tabular}{llll}
\hline & EC & MCC & At \\
\hline Water & $0.07 \pm 0.02$ & $2.76 \pm 0.04$ & $0.07 \pm 0.02$ \\
Water + ethanol & $0.23 \pm 0.01$ & $2.71 \pm 0.02$ & $0.64 \pm 0.14$ \\
\hline
\end{tabular}

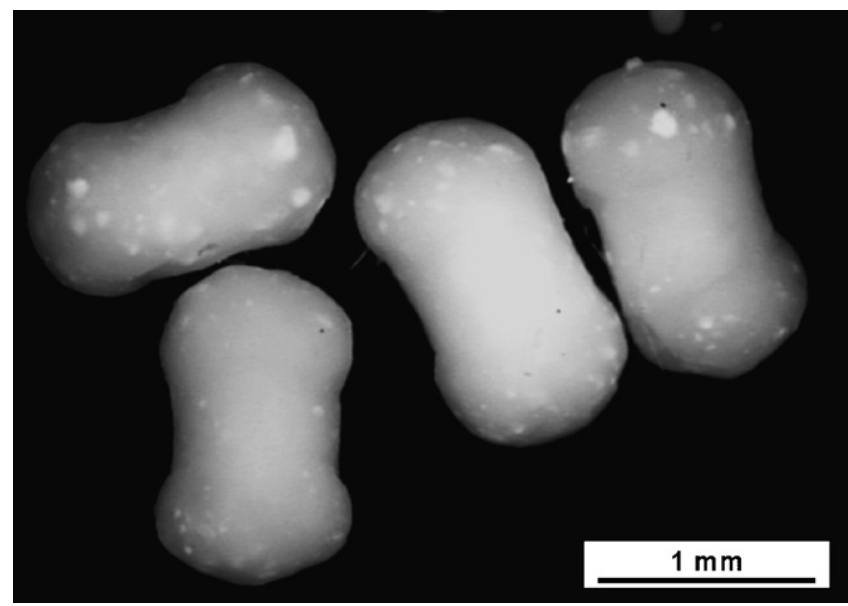

Fig. 1. S2 particles.

Statistica for Windows 8.1 AGA software (StatSoft, Inc. Tulsa, USA) was used for the calculations. 500 pellets of each sample were checked. During the mathematical evaluations, the confidence interval was $95 \%$, i.e. the differences were significant if $p<0.05$.

\section{Results and discussion}

\subsection{Wetting of powders}

Before the wetting of a powder mixture, it is important to know the liquid uptake of the powder mixture. This test was performed for both wetting liquids applied in this study. The highest sensitivity to the nature of the liquid was detected for At (Table 3). EC is soluble in ethanol, and the uptake for the liquid containing ethanol was also higher for EC. The lowest alteration was detected for MCC. It can be seen that the wettability of powders with water was negligible for the components other than MCC. In this case, the component mainly responsible for the structure-forming of the pellet must therefore be MCC. For the mixture of ethanol and water, not only the wetting of this component must be considered. In this case, the effects of other components (swelling of EC, recrystallization of At, etc.) can change the structure formed by MCC.

\subsection{Parameters of pellets}

The shapes of the pellets were very different for the different samples (Table 4 and Figs. 1 and 2). Better results (lower values of the aspect ratio), i.e. nearly spherical products, were observed for the particles prepared with water.

The situation was similar for the breaking hardness of the samples (Table 4). The evaluation of the process of pellet deformation involved not only determination of the breaking hardness, but also study of the deformation curve. The shapes of the breaking curves of the pellets were very similar for the various samples (Fig. 3). They mainly comprised three phases: a short elastic part, followed by a plastoelastic phase, and finally an elastic section peaking at the breaking point. The effect of the residual solvent on the mechanical properties of the spheres was neglected, because it was less than $0.5 \%$ for every sample. 
Table 4

Parameters of different pellets.

\begin{tabular}{|c|c|c|c|c|c|c|}
\hline Sample & Aspect ratio & Breaking hardness $(\mathrm{N})$ & Dissolution at $30 \mathrm{~min}(\%)$ & Breadth $(\mu \mathrm{m})$ & Length $(\mu \mathrm{m})$ & Water content (\%) \\
\hline S1 & $1.54 \pm 0.28$ & $12.00 \pm 1.80$ & $37.55 \pm 2.54$ & $1491.4 \pm 142.6$ & $2303.6 \pm 484.1$ & 0.23 \\
\hline $\mathrm{S} 2$ & $1.75 \pm 0.40$ & $13.84 \pm 2.00$ & $31.46 \pm 5.30$ & $1430.3 \pm 118.2$ & $2519.1 \pm 643.3$ & 0.27 \\
\hline S3 & $1.61 \pm 0.32$ & $12.20 \pm 1.76$ & $36.91 \pm 3.72$ & $1439.6 \pm 93.0$ & $2326.9 \pm 502.1$ & 0.30 \\
\hline S4 & $1.38 \pm 0.19$ & $11.70 \pm 1.89$ & $51.06 \pm 2.43$ & $1478.8 \pm 69.2$ & $2035.8 \pm 281.9$ & 0.23 \\
\hline S5 & $1.33 \pm 0.16$ & $12.68 \pm 1.19$ & $66.70 \pm 5.64$ & $1450.7 \pm 64.7$ & $1928.3 \pm 274.8$ & 0.27 \\
\hline S6 & $1.55 \pm 0.27$ & $15.53 \pm 1.71$ & $67.97 \pm 2.50$ & $1430.3 \pm 102.5$ & $2235.3 \pm 465.1$ & 0.40 \\
\hline S7 & $1.49 \pm 0.25$ & $13.55 \pm 1.69$ & $62.27 \pm 6.81$ & $1429.3 \pm 88.7$ & $2138.1 \pm 339.8$ & 0.23 \\
\hline S8 & $1.17 \pm 0.09$ & $12.45 \pm 1.38$ & $54.13 \pm 5.66$ & $1455.7 \pm 50.2$ & $1705.3 \pm 155.9$ & 0.23 \\
\hline
\end{tabular}

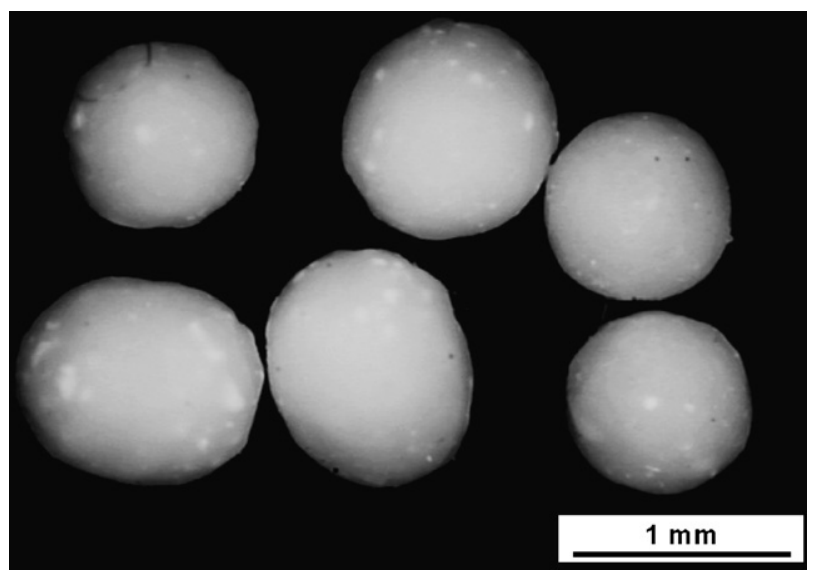

Fig. 2. S8 particles.

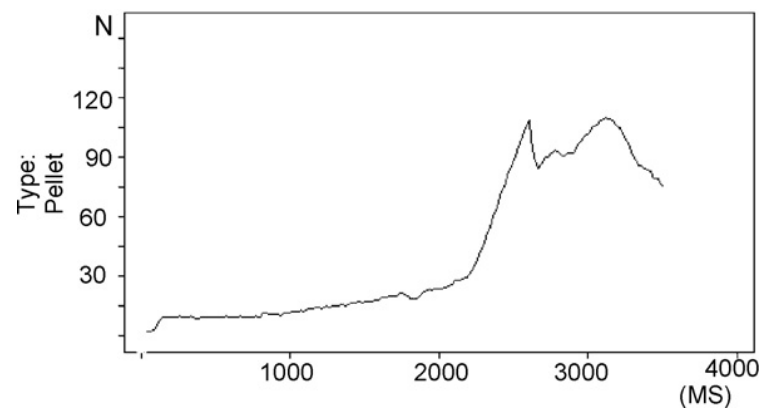

Fig. 3. Curve of pellet deformation.

The release of the active agent was sensitive to the nature of the kneading liquid. It was slower for the samples prepared with the mixture than for the samples prepared only with water (Table 4). The curves were nearly linear, which is suitable for our aim, but a few samples exhibited a step in the first phase (burst effect) which must be avoided (Fig. 4).

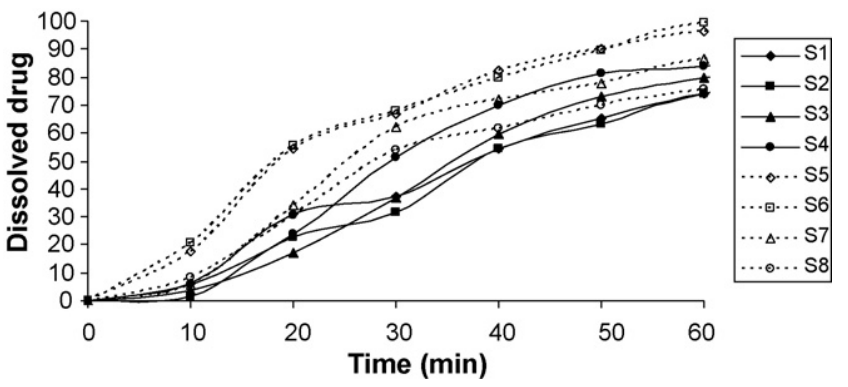

Fig. 4. Curves of pellet dissolution.

\subsection{Evaluation of the effects of factors}

A linear approach was applied for the fitting, and the correlation coefficient $\left(R^{2}\right)$ was very good in every case (Table 5). The duration of spheronization was found to be a significant factor as concerns the shape of the pellets. Its negative value indicated that increase of the duration enhanced the shape of the particles. The presence of ethanol in the wetting liquid destroyed the efficiency of the spheronization process.

The duration of spheronization was also significant for the breaking hardness of the spheres. The two other factors were similarly important. Increase of the value of each of the factors caused a decrease in this parameter. It is known that the shape can modify the breaking hardness of different systems (mainly for tablets) [41,42]. In the present case, there was no obvious connection between the relevance of the factors for shape and breaking. Modification of the internal structure of the spheres must therefore be responsible for this phenomenon. Formation of a mass with plasticity appropriate for spheronization was not possible with the liquid containing ethanol. Suitable wetting of MCC could not be achieved with the liquid with this composition. The squeezing of this liquid during spheronization can also be quicker, and thus the formation of an adequate structure, which is formed by MCC, was also disturbed. This process was enhanced by high speed and the duration of spheronization, which can be the explanation of the negative sign of these factors.

The presence of ethanol was most important for the dissolution at $30 \mathrm{~min}$. The effects of the other two factors were then negligible. In this case, the change in the shape cannot be the sole explanation of this alteration. This observation can again be explained by the changes induced in the internal structure by the different wetting liquids. The wetting of EC was different, so its different matrix-forming behaviour was induced by the ethanol. The presence of this type of matrix generated a greater alteration in the dissolution than in the breaking hardness. The explanation of this phenomenon was that the relevance of MCC in the modification of dissolution from an inert matrix is less than that in the mechanical properties of spheres. The effect of EC can therefore predominate.

Table 5

Values of coefficients.

\begin{tabular}{lccc}
\hline Factor & $\begin{array}{l}\text { Coefficient for } \\
\text { aspect ratio }\end{array}$ & $\begin{array}{l}\text { Coefficient for } \\
\text { breaking hardness }\end{array}$ & $\begin{array}{c}\text { Coefficient for } \\
\text { dissolution }\end{array}$ \\
\hline Intercept & 1.4763 & 12.9913 & 50.6838 \\
(1) Ethanol & 0.0913 & -0.5588 & -11.4413 \\
(2) Speed & -0.0663 & -0.5188 & -0.2363 \\
(3) Duration & $-0.1238^{*}$ & $-0.7888^{*}$ & 1.0313 \\
1 by 2 & -0.0113 & 0.0363 & 4.9738 \\
1 by 3 & 0.0113 & 0.2013 & 4.0263 \\
2 by 3 & -0.0163 & 0.3863 & -0.1738 \\
$R^{2}$ & 0.9973 & 0.9983 & 0.9731 \\
\hline
\end{tabular}

Significant $(p<0.05)$ 


\section{Conclusions}

It can be concluded that extrusion and spheronization comprised an appropriate method for the production of pellets from an EC and MCC mixture, but significant effects were exerted not only by the operational parameters, but also by the nature of the wetting liquid. Besides the shape, the internal structure of the pellets was changed by the optimum combination of factors, as indicated by the mechanical properties of the spheres and the dissolution of the active agent. The presence of ethanol in the liquid caused different degrees of wetting in the different components of the powder mixture. This appears can change the swelling of EC, and hence the formation of the matrix can vary. This alteration appears preferable in the dissolution, because the possibility of the burst effect in the first phase of dissolution can then be avoided. However it is not favourable as regards the shape and the mechanical properties of the pellets. This step of our work helped in the design of pellets containing an alkalizing component for increase of the bioavailability of the active agent. Application of ethanol is necessary, because of its favourable influence on the avoidance of a burst effect. However, it has negative effects on the shape and hardness. They can be enhanced by the use of an intensive spheronization process. Comparative studies of the wetting and the breaking hardness of pellets can facilitate the understanding of the process of pelletization optimization with the factorial design.

\section{Acknowledgements}

The authors wish to express their gratitude to Colorcon Ltd., Dartford, England, for the supply of ethylcellulose samples.

\section{References}

[1] T. Bussemer, I. Otto, R. Bodmeier, Pulsatile drug-delivery systems, Crit. Rev. Ther. Drug Carrier Sys. 18 (2001) 433-458.

[2] T. Bussemer, R. Bodmeier, Review of pulsatile drug delivery, Am. Pharm. Rev. 4 (2001) 24.

[3] B. Lemmer, Chronopharmacokinetics: implications for drug treatment, J. Pharm. Pharmacol. 51 (1999) 887-890.

[4] W.A. Ritschel, H. Forusz, Chronopharmacology: a review of drugs studies, Meth. Find. Exp. Clin. Pharmacol. 16 (1994) 57-75.

[5] J. Krämer, H. Blume, Biopharmaceutical aspects of multiparticulate, in: I. Ghebre-Selassie (Ed.), Multiparticulate Oral Drug Delivery, Marcel Dekker, New York, 1994, pp. 307-332.

[6] H. Bechgaard, N.G. Hagermann, Controlled-release multi-units and single unit doses. A literature review, Drug. Dev. Ind. Pharm. 4 (1978) 53-67.

[7] M. Bergeron, P. Laurin, R. Tawashi, Effect of particle morphology in selecting pharmaceutical excipients, Drug Dev. Ind. Pharm. 12 (1986) 915-926.

[8] P.J. Barret, The shape of rock particles, a critical review, Sedimentology 27 (1980) 291-303.

[9] J.M Newton, Extrusion and Extruders, in: J. Swarbrick, J.C. Boylan (Eds.), Encyclopedia of Pharmaceutical Technology, Marcel Dekker Inc., New York and Basel, 2002, pp. 1220-1236.

[10] R.D. Shah, M. Kabadi, D.G. Pope, L.L. Augsburger, Physicomechanical characterization of the extrusion-spheronization process. 2. Rheological determinants for successful extrusion and spheronization, Pharm. Res. 12 (1995) 496-507.

[11] J. Berggren, G. Alderborn, Effect of drying rate on porosity and tableting behaviour of cellulose pellets, Int. J. Pharm. 227 (2001) 81-96.

[12] Y.S. Habib, R.F. Shangraw, Effect of different drying techniques on the physicomechanical properties of beads containing microcrystalline cellulose produced by extrusion and spheronization, Pharm. Res. 14 (1997) S14.

[13] P. Kleinebudde, Shrinking and swelling properties of pellets containing MCC and low substituted hydroxy propyl cellulose. Part I. Shrinking properties, Int. J. Pharm. 109 (1994) 209-219.

[14] A.M. Dyer, K.A. Khan, M.E. Aulton, Effects of the drying method on mechanical and release properties of pellets prepared by extrusion-spheronization, Drug Dev. Ind. Pharm. 20 (1994) 3045-3068.
[15] B. Bataille, K. Ligarski, M. Jacob, C. Thomas, C. Duru, Study of the influence of spheronisation and drying conditions on the physico-chemical properties of neutral spheroids containing Avicel PH101 and lactose, Drug Dev. Ind. Pharm. 19 (1993) 653-671.

[16] D. Sonaglio, B. Bataille, C. Ortigosa, M. Jacob, Factorial design in the feasibility of producing Microcel MC 101 pellets by extrusion/spheronisation, Int. J. Pharm. 115 (1995) 53-60.

[17] Z.-W. Ye, P. Rombout, J.P. Remon, C. Vervaet, G. van den Mooter, Correlation between the permeability of metoprolol tartrate through plasticized isolated ethylcellulose/hydroxypropylmethylcellulose films and drug release from reservoir pellets, Eur. J. Pharm. Biopharm. 67 (2007) 485-490.

[18] J.W. Wallace, Cellulose derivatives and natural products utilized in pharmaceutics, in: J. Swarbrick, J.C. Boylan (Eds.), Encyclopedia of Pharmaceutical Technology, vol. 2, Marcel Dekker, New York and Basel, 2002.

[19] R.M. Iyer, L.L. Augsburger, D.M. Parikh, Evaluation of drug layering and coating: effect of process mode and binder level, Drug Dev. Ind. Pharm. 19 (1993) 981-998.

[20] C.C. DeMerlis, D.R. Schoneker, J.F. Borzelleca, A subchronic toxicity study in rats and genotoxicity tests with an aqueous ethylcellulose dispersion, Food Chem. Toxicol. 43 (2005) 1355-1364

[21] K. Naelapää, P. Veski, H.G. Kristensen, P. Bertelsen, Coating of potassium chloride with ethylcellulose aqueous dispersion-effects of processing parameters on film formation and film properties, Eur. J. Pharm. Sci. 32 (2007) S20.

[22] R.C. Rowe, P.J. Sheskey, P.I. Weller, Handbook of Pharmaceutical Excipients, fourth ed., Pharmaceutical Press, London, 2003.

[23] K.H. Bauer, K. Lehmann, H.P. Osterwald, G. Rothang, Coated Pharmaceutical Dosage Forms, Medpharm GmbH Scientific Publishers, Stuttgart, 1988.

[24] G.G. Briggs, R.K. Freeman, S.J. Yaffe, Drugs in Pregnancy and Lactation, Fourth edition, Williams and Wilkins, Baltimore, 1994.

[25] J.R. Niebyl, Teratology, Drugs in Pregnancy and Lactation, in: T. Scott (Ed.), Danforth's Obstetrics and Gynecology, 6th ed., Lipincott, Philadelphia, 1990.

[26] J.D. Fitzgerald, The biological and clinical effects of atenolol (Tenormin) a cardioselective-antagonist, in: M.E. Goldberg (Ed.), Pharmacological and Biochemical Properties of Drug Substances, vol. II, American Pharmaceutical Association Press, Washington DC, 1979.

[27] F.D. Thompson, Review of clinical pharmacology of atenolol, Proc. R. Soc. Med. 70 (Suppl. 5) (1977) 5-8

[28] S.A.Tabacova, C.A. Kimmel, Atenolol: pharmacokinetic/dynamic aspects of comparative developmental toxicity, Reprod. Toxicol. 16 (2002) 1-7.

[29] K. Karin, H. Jyrki, V. Johanna, M. Martti, A. Osmo, Y. Jouko, Extrusion-spheronization of $\mathrm{pH}$-sensitive polymeric matrix pellets for possible colonic drug delivery, Int. J. Pharm. 199 (2000) 187-194.

[30] A.M. Ketan, M.S. Kislalioglu, W. Phuapradit, A.W. Malick, N.H. Shah, Effect of formulation and process variables on porosity parameters and release rates from a multi unit erosion matrix of a poorly soluble drug. J. Control. Release 63 (2000) 201-211.

[31] G. Tomer, F. Podczeck, J.M. Newton, The influence of model drugs on the preparation of pellets by extrusion/spheronization: II spheronization parameters, Int J. Pharm. 231 (2002) 107-119.

[32] E. Nürnberg, P. Surmann (Eds.), Hagers Handbuch der pharmazeutischen Praxis, fifth edition, Springer Verlag, Bd. 2 Methoden, Berlin, New York, 1991, p. 60

[33] S.A. de Assis, B.S. Ferreira, P. Fernandes, D.G. Guaglianoni, J.M.S. Cabral, M.F. Oliveira, Gelatin-immobilized pectinmethylesterase for production of low methoxyl pectin, Food Chem. 86 (2004) 333-337.

[34] J. Todorovic, H. Ecke, A. Lagerkvis, Solidification with water as a treatment method for air pollution control residues, Waste Manage. 23 (2003) 621-629.

[35] M.M.C. Forte, F.O.V. Cunha, J.H.Z. Santos, J.J. Zacca, Ethylene and 1-butene copolymerization catalyzed by a Ziegler-Natta/metallocene hybrid catalyst through a 23 factorial experimental design, Polymer 44 (2003) 1377-1384.

[36] J. Bajdik, G. Baki, A. Kelemen, K. Pintye-Hódi, The effect of wetting of a powder mixture on the preparation of hydrophilic matrix granules with a high-shear granulator, Chem Eng. Res. Des. 86 (2008) 1-7.

[37] M.C. Gohel, M.M. Patel, A.F. Amin, Development of modified release diltiazem $\mathrm{HCl}$ tablets using composite index to identify optimal formulation, Drug Dev. Ind. Pharm. 29 (2003) 565-574.

[38] G. Sertsou, J. Butler, A. Scott, J. Hempenstall, T. Rades, Factors affecting incorporation of drug into solid solution with HPMC during solvent change co-precipitation, Int. J. Pharm. 245 (2002) 99-108.

[39] K.H. Khanvilkar, Ye Huang, A.D. Moore, Influence of hydroxypropyl methylcellulose mixture, apparent viscosity, and tablet hardness on drug release using $2^{3}$ full factorial design, Drug Dev. Ind. Pharm. 28 (2002) 601-608.

[40] B. Davies, S. Ellis, Process analytical technology: the route to process understanding and control, Pharma. Technol. Eur. 17 (2005) 17-23.

[41] P.N. Davies, H.E.C. Worthington, F. Podczeck, J.M. Newton, The determination of the mechanical strength of tablets of different shapes, Eur. J. Pharm. Biopharm. 67 (2007) 268-276.

[42] E. van Santen, D.M. Barends, H.W. Frijlink, Breaking of scored tablets: a review, Eur. J. Pharm. Biopharm. 53 (2002) 139-145. 


\section{III.}




\title{
Study of the influence of alkalizing components on matrix pellets prepared by extrusion/spheronization
}

\author{
Elnazeer I. Hamedelniel, János Bajdik, Péter Kása Jr, and Klára Pintye-Hódi \\ Department of Pharmaceutical Technology, University of Szeged, Szeged, Hungary
}

\begin{abstract}
The aim of this study was to investigate the effects of alkalizing components and the nature of the wetting liquid on the properties of matrix pellets prepared by extrusion and spheronization. Atenolol was used as an active pharmaceutical ingredient, ethylcellulose as a matrix former, microcrystalline cellulose as a filler and disodium phosphate anhydrous and trisodium phosphate dodecahydrate as alkalizing materials. Water and a water-ethanol mixture served as granulation liquids. Pellet formation was evaluated via mechanical, dissolution and morphological studies. In order to enhance the dissolution of Atenolol from the pellets, alkalizing components were used and the influence of these components on the $\mathrm{pH}$ was tested. Investigations of the breaking hardness, the morphology and the dissolution revealed that the pellets containing trisodium phosphate dodecahydrate and prepared with a higher amount of water as binding liquid displayed the best physico-chemical parameters and uniform dissolution. In in vitro experiments, the dissolution release complied with the texture of the pellets and the effect of $\mathrm{pH}$. The pellets have suitable shape and very good hardness for the coating process and are appropriate for subsequent in vivo experiments.
\end{abstract}

Keywords: Matrix pellets, alkalizing components, extrusion, spheronization

\section{Introduction}

The pelletization process consists of the agglomeration of fine powders of active ingredient(s) and excipients into small spherical units. Pellets are commonly prepared by applying the extrusion/spheronization pelletization process. This comprises five unit operations: Blending, wet massing, extrusion, spheronization and drying. The extrusion of a wet powder mass leads to an intermediate spaghetti-like product, which is promptly spheronized to yield a final spherical product. ${ }^{[1,2]}$ Since these phases are strongly related to each other, ${ }^{[3]}$ the quality of the endproduct (pellets) is also strongly dependent on the process factors. ${ }^{[4]}$ The physical characteristics of the pellets, which are directly affected by the process and formulation variables, ${ }^{[-8-8]}$ highly influence the further manufacturing processes, such as film coating, capsule filling or tabletting, and consequently the properties of the final dosage form and its biological performance.

Atenolol (At) is a potent cardioselective $\beta$-adrenoreceptor blocking agent ${ }^{[9]}$ used for the treatment of hypertension, including hypertension in pregnancy. ${ }^{[10,11]}$ Following oral administration, the absorption of the drug in humans and most laboratory animal species is rapid, but incomplete (50-60\%). It has been shown that Atenolol is well absorbed at $\mathrm{pH}>7.5 .^{[12]}$ The normal $\mathrm{pH}$ of the human ileum is 7.2-7.6, and thus absorption is not total. When adminsterd intravenously, Atenolol is excreted almost completely in the urine. Following oral administration, between $40 \%$ and $50 \%$ of the unchanged compound is recovered in the urine, and $50 \%$ is recovered unchanged in the faeces, due to incomplete intestinal absorption in the human and in most experimental species, except for the dog, in which the $\mathrm{pH}$ of the ileum is $>8$, and in which absorption from its gut is therefore almost complete: $98 \%$ of the total dose. ${ }^{[13]}$ This striking difference in intestinal absorption has been attributed to the interspecies differences in intestinal luminal $\mathrm{pH}$. There is no evidence of an alternative biliary route for excretion in either humans or experimental animals. ${ }^{[1,15]}$ As Atenolol is well absorbed at $\mathrm{pH}>7.5{ }^{[12]}$ 
it must be administrered with a component that ensures a suitable local $\mathrm{pH}$. Preparation of a multiparticulate matrix system with an alkalizing component is a reasonable way to ensure an appropriately alkaline micromilieu. A matrix former polymer is required to control the parallel release of Atenolol and the alkalizing component utilized to produce sustained-release dosage forms with good bioavailability.

Atenolol may be incorporated into an insoluble matrix. The penetration of fluid into this system must precede the drug release from such a matrix. Fluid penetration is followed by dissolution of the drug particles and diffusion through fluid-filled pores. The excipients used in the preparation of insoluble matrices include inert hydrophobic polymers, such as ethylcellulose (EC), polyvinyl acetate, acrylic derivatives, etc.

EC has frequently been used as a coating material for tablets and pellets ${ }^{[16-18]}$ and as a binder in the preparation of microcapsules and microspheres, but it is also used as monolithic matrix former for formulations in which modified drug release is ensured by diffusion. ${ }^{[19-21]}$

As mentioned above, extrusion/spheronization is nowadays a frequently used method for the preparation of pellets. For successful extrusion and spheronization, Shah et al. ${ }^{[22]}$ incorporated microcrystalline cellulose (MCC) in many formulations, since it provides the wetted mass with the appropriate rheological properties. ${ }^{[23]}$ In order to obtain a very good mass for this process, it is necessary to use MCC and the matrix former together.

During the processes, it is very important to ensure the best rheological properties for the wet mass. Water is generally used as wetting liquid. Since EC dissolves in ethanol, it was interesting to study the effects of ethanol or an ethanol-water mixture on the rheological properties of the wet mass and the final product. For this reason, the aim of the present study was to investigate the influence of the alkalizing components and the wetting liquid on the formulation of pellets containing Atenolol, prepared via extrusion/spheronization, and on their dissolution.

\section{Materials and methods}

\section{Materials}

Atenolol (At) (Ariane Organochem Private Ltd, Mumbai, India) was applied as model drug, ethyl cellulose (EC)
(Ethocel standard 10 premium, Colorcon Ltd, Dartford, Kent, UK) as matrix former and microcrystalline cellulose (MCC) (Vivapur 103, Rettenmaier \& Söhne GmbH, Rosenberg, Germany) as spheronization enhancer. Trisodium phosphate dodecahydrate $\left(\mathrm{Na}_{3} \mathrm{PO}_{4} * 12 \mathrm{H}_{2} \mathrm{O}\right)$ (VWR International, Belgium) and disodium phosphate anhydrous $\left(\mathrm{Na}_{2} \mathrm{HPO}_{4}\right)$ (Spektrum 3DKF Debrecen, Hungary) were used as alkalizing agents. Ethanol $96 \%$ (Spectrum-3D, Debrecen, Hungary) as a $16.3 \% \mathrm{v} / \mathrm{v}$ mixture with water or water alone served as wetting components.

\section{Preparation of pellets}

$150 \mathrm{~g}$ of powder mixture was prepared from $37.5 \mathrm{~g}$ of Atenolol, $37.5 \mathrm{~g}$ of alkalizing component (either disodium hydrogenphosphate or trisodium phosphate), $45 \mathrm{~g}$ of MCC and $30 \mathrm{~g}$ of EC. To obtain a uniform mixture, the powder was blended at $50 \mathrm{rpm}$ for $10 \mathrm{~min}$ with a Turbula mixer (W.A. Bachofen, Basel, Switzerland) (Table 1).

Wetting was performed in a high-shear mixer (ProCepT 4M8 granulator, ProCepT nv, Zelzate, Belgium). Kneading was carried out with the aid of a granulating liquid $(85 \mathrm{~mL}$ or $75 \mathrm{~mL}$ ), consisting of water alone or a water-ethanol mixture (16.3\% v/v ethanol in water). Eight samples of pellets were prepared by extrusion/spheronization methods. The amount of ethanol used and the parameters of the preparation were based on the results of previous work. ${ }^{[24]}$

The kneading parameters were as follows: Impeller speed $1500 \mathrm{rpm}$, chopper speed $2000 \mathrm{rpm}$ and dosing speed $5 \mathrm{~mL} / \mathrm{min}$. The kneading process was followed by the software incorporated in the apparatus. The curve on the monitor reveals the most important parameters (temperature and the torque of the impeller) for the best mass.

The wet mass obtained was extruded by a mini-screw (Caleva Ltd, Sturminster Newton, Dorset, UK) equipped with an axial screen with dies $1 \mathrm{~mm}$ in diameter and $4 \mathrm{~mm}$ in length, operating at $90 \mathrm{rpm}$. The jacked barrel of the extruder was cooled by water at $25 \pm 2^{\circ} \mathrm{C}$. Each extrudate was spheronized on a spheronizer $12 \mathrm{~cm}$ in diameter (Model-120, G.B. Caleva Ltd, Sturminster Newton, Dorset, UK) fitted with a cross-hatch grooved plate, for $10 \mathrm{~min}$ at $1000 \mathrm{rpm}$. The pellets were dried under the same conditions, at $40 \pm 2^{\circ} \mathrm{C}$ for $24 \mathrm{~h}^{[24]}$

Table 1. Compositions of samples.

\begin{tabular}{|c|c|c|c|c|c|c|c|c|}
\hline \multirow[b]{2}{*}{ Samples } & \multicolumn{5}{|c|}{ Powder } & \multicolumn{3}{|c|}{ Binding liquid } \\
\hline & At $\%$ & MCC \% & EC \% & $\mathrm{Na}_{2} \mathrm{HPO}_{4} \%$ & $\mathrm{Na}_{3} \mathrm{PO}_{4} \%$ & Water \% & Ethanol \% & Total $\mathrm{mL}$ \\
\hline$\overline{\mathrm{P} 1}$ & 25 & 30 & 20 & 25 & - & 100 & - & 85 \\
\hline $\mathrm{P} 2$ & 25 & 30 & 20 & - & 25 & 100 & - & 85 \\
\hline P3 & 25 & 30 & 20 & 25 & - & 83.7 & 16.3 & 85 \\
\hline P4 & 25 & 30 & 20 & - & 25 & 83.7 & 16.3 & 85 \\
\hline P5 & 25 & 30 & 20 & 25 & - & 100 & - & 75 \\
\hline P6 & 25 & 30 & 20 & - & 25 & 100 & - & 75 \\
\hline $\mathrm{P} 7$ & 25 & 30 & 20 & 25 & - & 83.7 & 16.3 & 75 \\
\hline P8 & 25 & 30 & 20 & - & 25 & 83.7 & 16.3 & 75 \\
\hline
\end{tabular}




\section{Morphological study}

The particle size and the shape of the pellet surface were studied by using a system consisting of a stereomicroscope (Zeiss Stemi 2000-C, Carl Zeiss GmbH, Vienna, Austria) and a ring light with a cold light source (Leica KL 1500, Cambridge, UK). A Quantimet 500 (Q500MC) image processing and analysis system (Leica Cambridge Ltd, Cambridge, UK) was used. The aspect ratio was utilized for the evaluation of the shape of the particles.

\section{Mechanical properties of the pellets}

The breaking hardess tester and the software were developed in our institute. The tester contains a specimen holder and a jowl, and is connected to a computer via an interface. The specimen is located horizontally on a special plate and the jowl moves vertically. Twenty parallel measurements were performed for each pellet sample, independently of the aspect ratio. The measurement range was $0-200 \mathrm{~N}$, the speed of the stamp was $20 \mathrm{~mm} /$ min, and the output was $0-5 \mathrm{~V}$. The sensor was a Unicell force-measuring instrument, calibrated with the $\mathrm{C} 9 \mathrm{~B}$ $20 \mathrm{kN}$ cell.

\section{$\mathrm{pH}$ measurement}

$0.1 \mathrm{~g}$ of pellets was weighed and placed in a beaker containing $50 \mathrm{~mL}$ of distilled water or buffer $(\mathrm{pH}=6.8)$, which was agitated magnetically. A small amount of liquid was used for the testing to state the effect on the micromilieu. After each $10 \mathrm{~min}$ of stirring $(120 \mathrm{rpm})$, the pellets were filtered off and the $\mathrm{pH}$ of the solution was measured. To measure the changes in $\mathrm{pH}$ with time, the filtered pellets were again placed in $50 \mathrm{~mL}$ of distilled water or buffer. This process was repeated until the $\mathrm{pH}$ remained unchanged. Each test was repeated three times.

\section{Dissolution tests}

Pellets $(100 \mathrm{mg})$ were filled into gelatin capsules, which were placed in the basket of a dissolution tester (Erweka DT 700, Heusenstamm, Germany). The dissolution medium consisted of $900 \mathrm{~mL}$ of phosphate buffer $(\mathrm{pH}$ 6.8) maintained at $37.0 \pm 0.5^{\circ} \mathrm{C}$. The rotation speed of the baskets was set at $100 \mathrm{rpm}$. The dissolution system was combined with an automatic sampling station. Samples of $5 \mathrm{~mL}$ were withdrawn at $10,20,30,40,50$ and $60 \mathrm{~min}$ through the filter, which has pores $10 \mu \mathrm{m}$ in diameter. The dissolved Atenolol was measured spectrophotometrically (Unicam Helios Alpha, Spectronic Unicam, Cambridge, UK) at $\lambda_{\text {max }}=224 \mathrm{~nm}$

\section{SEM testing of pellets}

The surfaces of various samples and the internal structures of the pellets were tested with a scanning electron microscope (SEM) (Hitachi S4700, Hitachi Scientific Instruments Ltd, Tokyo, Japan). A SEM sputter coating unit (Polaron E5100, VG Microtech, UK) was used to make the surfaces conductive for the SEM measurements. The air pressure during the analyses was 1.3-13 $\mathrm{mPa}$. The accelerating voltage was in the range $7-15 \mathrm{kV}$.
The actual value and the magnification are detailed in the Figures.

\section{Results and discussion}

Table 2 reveals that the shapes of the different pellets differed considerably Sample P2 had the best aspect ratio (close to 1), which means that this sample, prepared with the higher amount of water and containing trisodium phosphate, had the best spherical form. For the pellets containing disodium phosphate anhydrous, the shape was not round and the surface was very rough. The aspect ratio was a little better when the higher amount of wetting liquid was used. Figure 1 demonstrates the non-spherical form and the roughness of the surface of such a sample (P1), and the higher magnification shows the sponge-like texture with numerous pores (Figure 2).

The formation and hence the shape and surface of the pellets depend on the consistency of the mass, which can influence the solubility of the components. Atenolol dissolves only sparingly in water, but is soluble in alcohol. According to the literature, the water solubilities of disodium phosphate anhydrous and trisodium phosphate dodecahydrate are very good, the latter dissolving better. Furthermore, these components are insoluble in alcohol. It is well known that the solubility of EC in alcohol is very good, and the dissolved EC would form films after drying. However, this is limited by the amount of granulating liquid and the ratio of the water-ethanol.

For the pellet containing MCC, the binder forces can be assigned to its 'crystallite-gel model. ${ }^{\text {[25] }}$ MCC particles are broken down into smaller units and even partly into single crystals of colloidal size during granulation and extrusion in the presence of water. The resulting crystallites and porous particles form a coherent gel-like network (with a high fraction of an insoluble solid phase) and immobilize the liquid. Over a particular range of water, which relates to acceptable gel strength, extrusion and spheronization become possible.

In the samples which contained trisodium phosphate and in which the wetting liquid was water alone in higher amount, the alkalizing component could take up the water from the powder mixture. This effect improves the plasticity of the wetted mass and enhances the spheronization, and is therefore a pellet structure-forming parameter which can determine not only the shape, but also the

Table 2. Shape parameters of pellets.

\begin{tabular}{lccc}
\hline Sample & Breadth $(\mathrm{mm})$ & Length $(\mathrm{mm})$ & Aspect ratio \\
\hline P1 & $1.24 \pm 0.19$ & $1.44 \pm 0.42$ & $1.32 \pm 0.54$ \\
P2 & $1.72 \pm 0.15$ & $1.88 \pm 0.16$ & $1.14 \pm 0.04$ \\
P3 & $1.55 \pm 0.16$ & $1.95 \pm 0.37$ & $1.22 \pm 0.36$ \\
P4 & $1.54 \pm 0.14$ & $1.68 \pm 0.22$ & $1.20 \pm 0.25$ \\
P5 & $1.59 \pm 0.22$ & $2.73 \pm 0.70$ & $1.43 \pm 0.36$ \\
P6 & $1.62 \pm 0.16$ & $1.99 \pm 0.33$ & $1.19 \pm 0.24$ \\
P7 & $1.67 \pm 0.26$ & $3.40 \pm 0.94$ & $1.61 \pm 0.34$ \\
P8 & $1.54 \pm 0.96$ & $1.92 \pm 0.24$ & $1.18 \pm 0.14$ \\
\hline
\end{tabular}


mechanical properties. During drying, rather fast recrystallization of the trisodium phosphate occurred, resulting in strong solid bridges with the other particles, e.g. MCC. The pellets were spherical and the surface was smooth, with some narrow or round pores, formed during drying (P2, Figure 3). It can be seen at higher magnification that the texture of the pellet was very compact (Figure 4), and the hardness of these pellets was very high. On reduction of the water amount, the degree of recrystallization also decreased, but in spite of this the hardness was very good (Table 3).

The SEM investigation allowed the effect of ethanol on the texture of the pellets to be followed. Partial dissolution and fast recrystallization of the Atenolol occurred in the mass. Some of the EC also partially dissolved in the ethanol and formed film pieces, which can be seen at higher magnification (Figure 5). On reduction of the wetting liquid amount (including that of the ethanol in it), only smaller films could form and the recrystallization resulted very little crystal aggregation (Figure 6). The solid bonds in the texture were therefore weakened somewhat. More pores could be seen in the texture and the hardness was a little less than that of P2 (Figure 3).

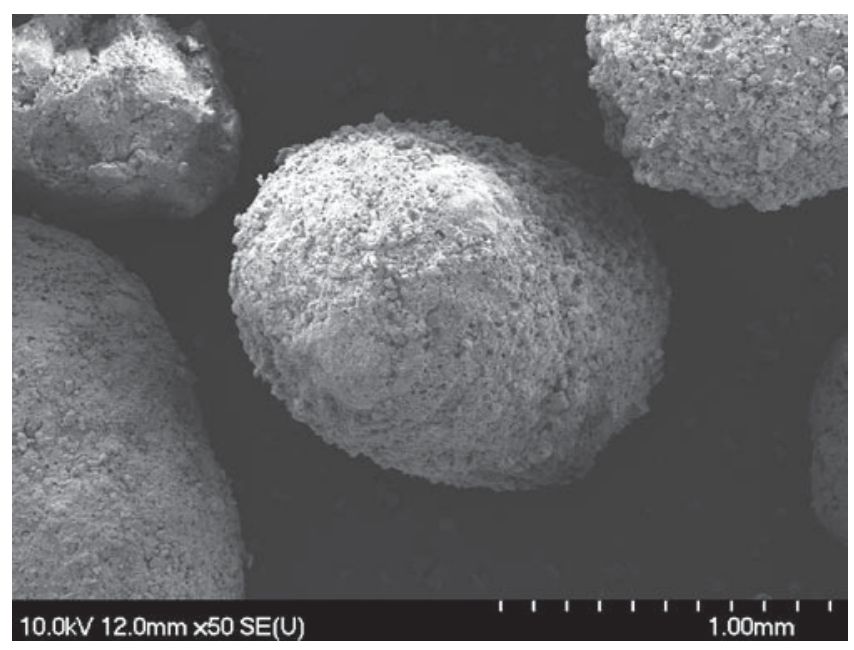

Figure 1. Shape of P1 sample.

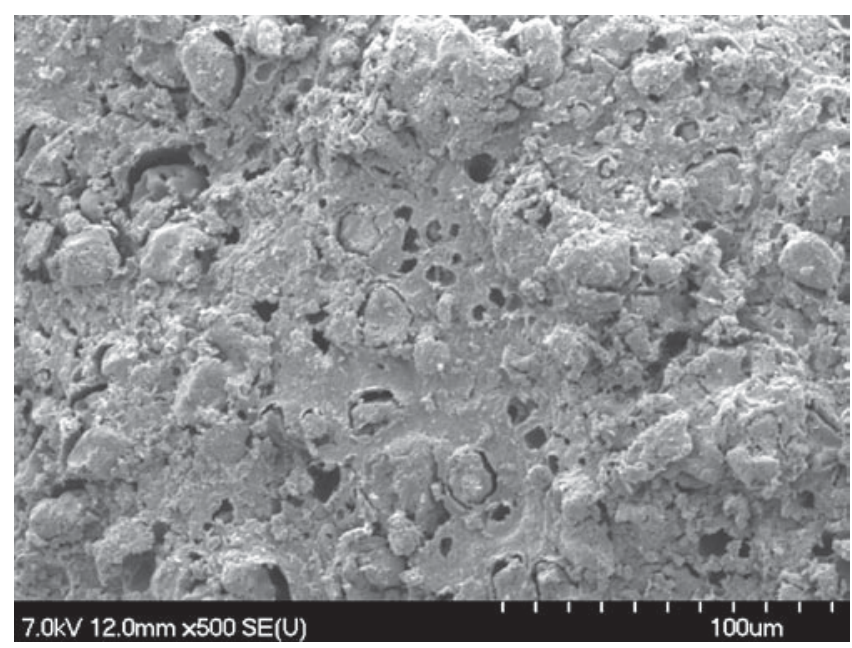

Figure 2. Detail of the surface of P1 sample.
However, it can be seen from the data in Table 3 that the pellets containing trisodium phosphate had very high hardness. Decrease of the amount of water or use of the water-ethanol mixture as wetting liquid reduced the hardness, but only slightly (P4, P6 and P8).

The texture of the pellets containing disodium phosphate anhydrous was generally looser. It can be seen in Figure 1 that the pellets prepared with water alone were non-spherical and very rough (P1). Higher magnification showed that the partial dissolution and fast recrystallization resulted in a sponge-like texture with many pores (Figure 2), and therefore a low breaking hardness. In the case of the smaller amount of water, the degree of recrystallization decreased and the texture revealed numerous bonds between the small crystals and particles (P5, Figure 7).

In the presence of ethanol, Atenolol and EC dissolved, resulting in recrystallized Atenolol aggregates and variously-sized pieces of EC film, as can be seen in the texture of P3 (Figure 8) in comparison with the SEM photo of the bulk Atenolol substance (Figure 9), which demonstrates the crystal habit. A large number of small crystals can be seen among the larger irregular-shaped

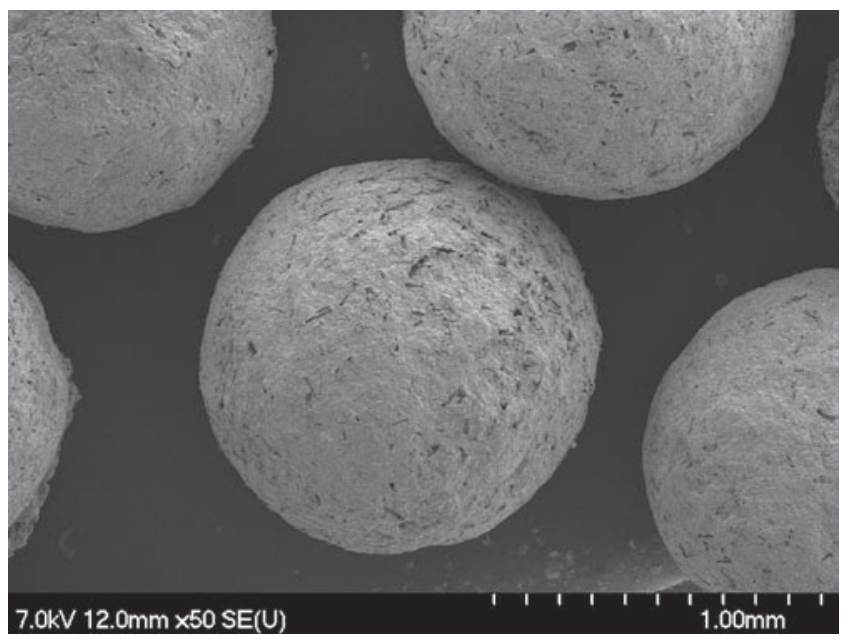

Figure 3. Shape of P2 pellets.

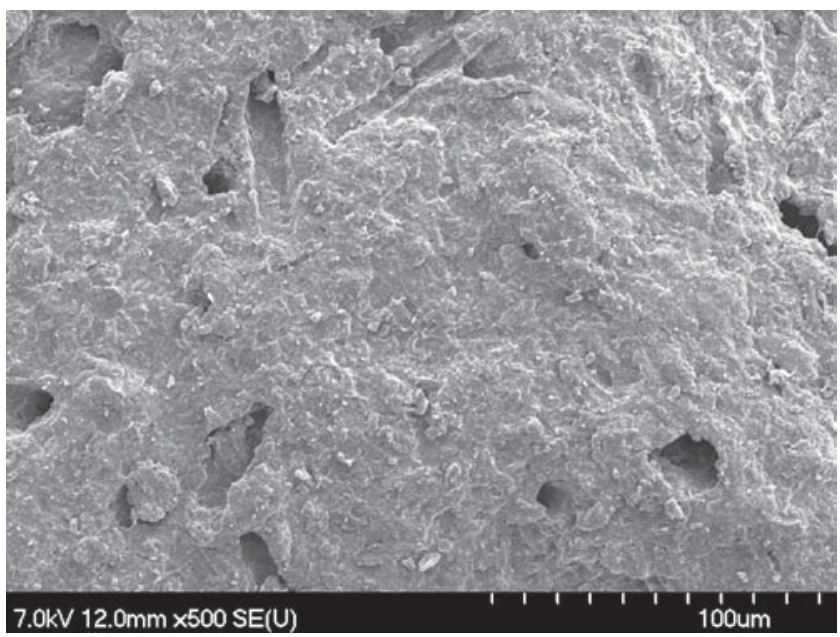

Figure 4. Detail of the surface P2 sample. 
Table 3. Breaking hardness of pellets.

\begin{tabular}{lcccccccc}
\hline Sample & P1 & P2 & P3 & P4 & P5 & P6 & P7 & P8 \\
\hline Breaking hardness (N) & $2.61 \pm 0.61$ & $12.28 \pm 2.03$ & $1.16 \pm 0.32$ & $9.84 \pm 1.82$ & $2.75 \pm 1.32$ & $9.09 \pm 1.78$ & $1.89 \pm 0.54$ & $9.14 \pm 3.09$ \\
\hline
\end{tabular}

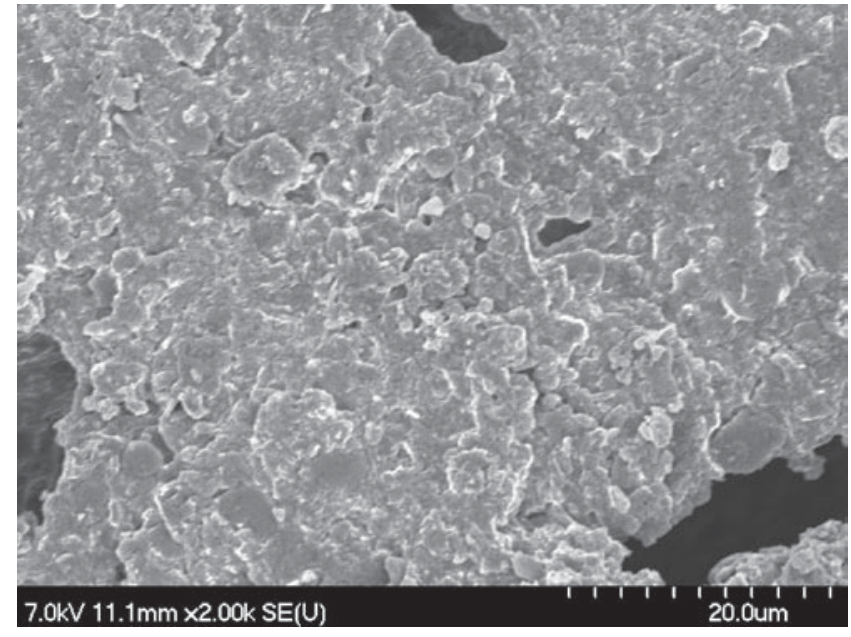

Figure 5. Detail of the surface P4 sample.

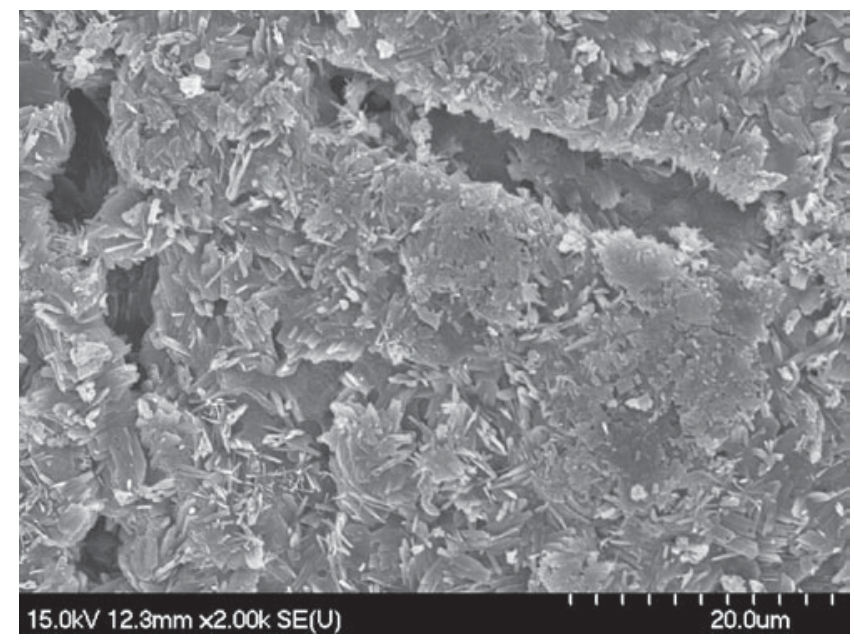

Figure 6. Detail of the surface of P8 sample.

crystals. This habit is in agreement with the habit of the small recrystallized particles in Figure 10. On decrease of the amount of the wetting liquid, the sponge-like matrix predominated, formed by the EC film with small Atenolol crystals spread on its surface (P7, Figure 10). As regards the pellets containing disodium phosphate anhydrous, it can be stated that the hardness of these pellets was practically independent of the wetting liquid (P1, P3, P5 and P7).

It was mentioned above that, from the aspects of suitable dissolution and bioavailability, the role of the $\mathrm{pH}$ in the intestine is very important. For this reason, the effect of the alkalizing component on the $\mathrm{pH}$ in the micromilieu was studied.

Tables 4 ( $a$ and $b$ ) illustrate the effects of the dissolved alkalizing components on the $\mathrm{pH}$ change. It can be seen that in water the $\mathrm{pH}$ in the micromilieu increased very rapidly, and this effect lasted $1 \mathrm{~h}$. After $10 \mathrm{~min}$, the $\mathrm{pH}$ was $>10$ in every case, independently

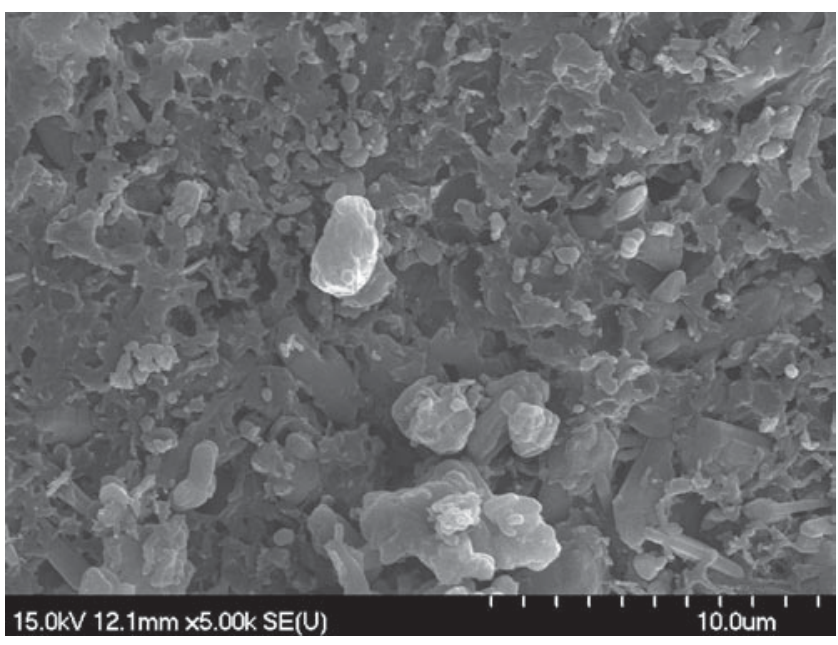

Figure 7. Detail of the surface of P5 sample.

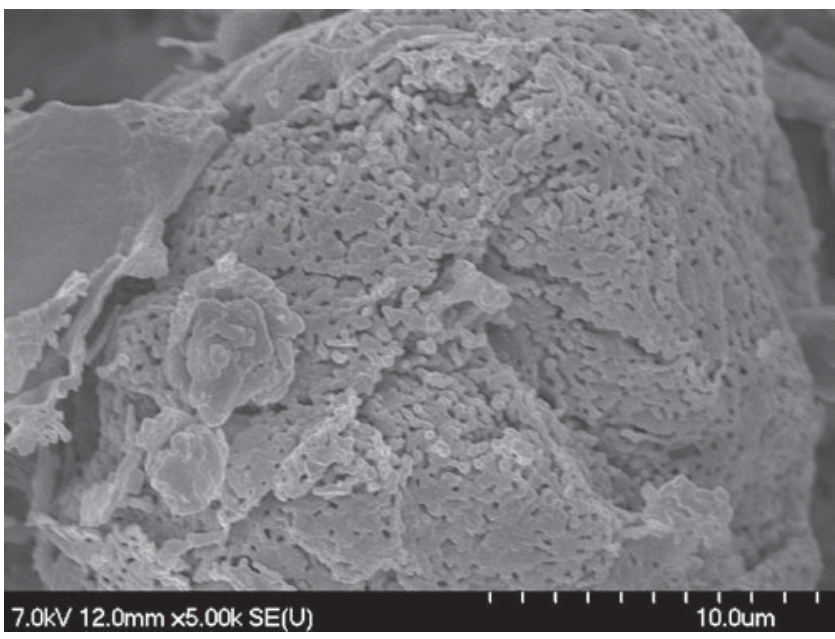

Figure 8. Detail of the surface P3 sample.

of the type of the pellets. After $20 \mathrm{~min}$, some difference could be seen, but it can generally be stated that, in the case of the trisodium phosphate-containing pellets, the $\mathrm{pH}$ exceeded 10 and decreased only gradually during time. The duration of the alkaline $\mathrm{pH}$ value was best for the $\mathrm{P} 2$ pellets.

The effects of the dissolved alkalizing components can be seen in buffer solution too, but naturally to a smaller degree (Table $4 \mathrm{~b}$ ). After $10 \mathrm{~min}$, the $\mathrm{pH}$ in the micromilieu had increased from 6.8 to 7.5-7.8. The duration of this effect was best in the case of P1 and P2, where the granulating liquid was water alone. These results suggest the better dissolution of Atenolol.

The in vitro dissolution test generally revealed a tendency to sustained-release and the alkalizing micromilieu enhanced the dissolution of Atenolol from the matrix. The dissolution profiles displayed a sigmoid shape, which is characteristic of sustained release preparations. 
However the dissolution from the samples containing disodium phosphate anhydrous was very uneven (P1, P3, P5 and P7) and the deviation was high (sometimes more than 20\%). The reason for this lies in the very loose texture with large high pores and the too weak bonds. Accordingly, these pellets were omitted from the further experiments.

The process of dissolution from the pellets containing trisodium phosphate (P2, P4, P6 and P8) was better, and the deviation (SE) was smaller (Figure 11). The best deviation was observed for the P2 and P4 pellets, when the wetting liquid was used in higher amount. For pellets P6 and P8, where the amount of wetting liquid was lower, the deviation was higher. These results are in accordance with the texture of the pellets. The most uniform dissolution was observed for sample P2, where

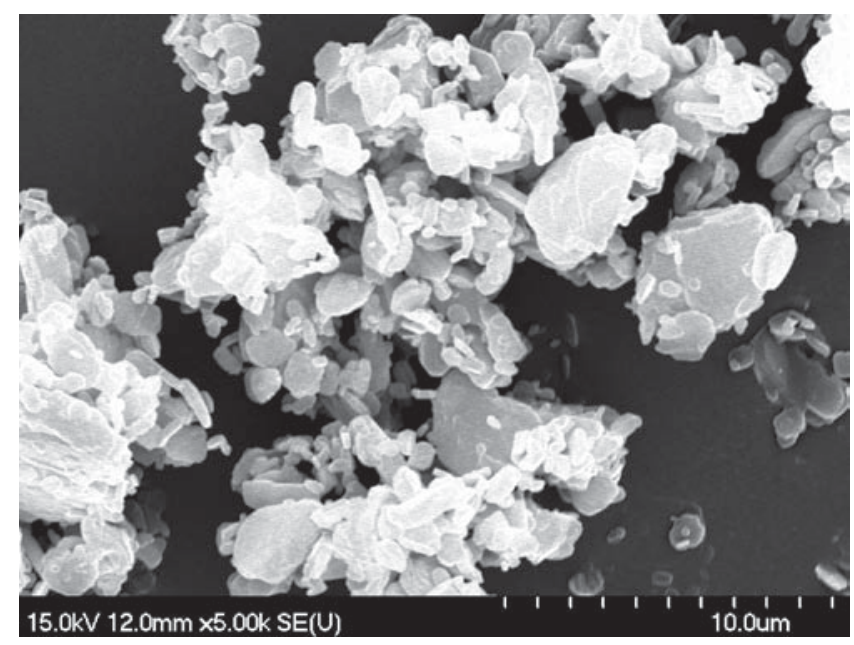

Figure 9. Atenolol crystals. the alkalizing agent was trisodium phosphate and the binding liquid was the higher amount of water. The texture of this pellet was very compact, and it had very high hardness.

In the final dosage form, the pellets containing Atenolol should be coated with intestine-soluble polymer. The dissolution results, in accordance with the morphological observations and the hardness of the pellets, indicate that the $\mathrm{P} 2$ pellets have the best composition, and are suitable for the development of the final dosage form.

\section{Conclusions}

In order to enhance the bioavailability of Atenolol from pellets, alkalizing components (disodium phosphate

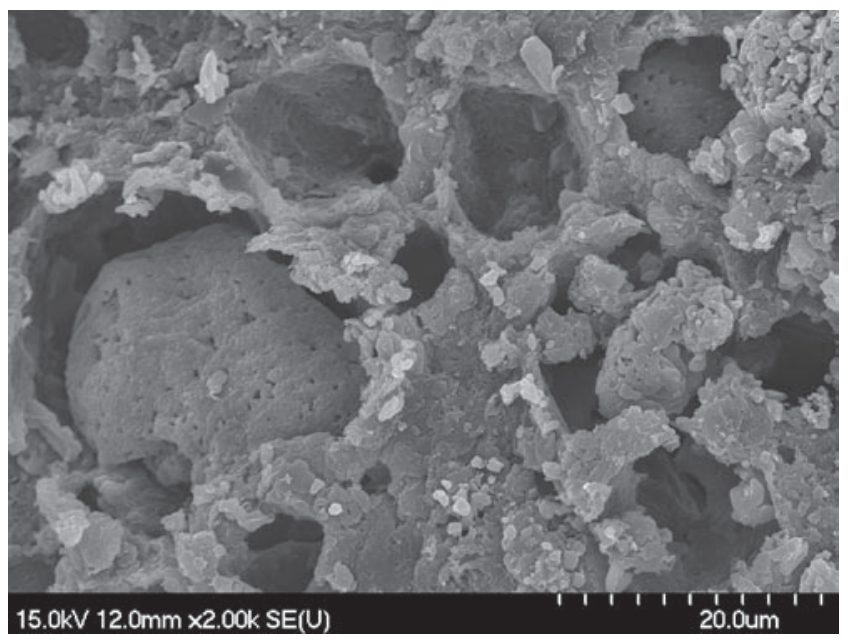

Figure 10. Detail of the surface P7 sample.

Table 4a. Influence of pellets on the $\mathrm{pH}$ of solution.

\begin{tabular}{|c|c|c|c|c|c|c|c|c|}
\hline \multirow[b]{2}{*}{ Sample } & \multicolumn{8}{|c|}{$\mathrm{pH} /$ time $(\mathrm{min})$ in water } \\
\hline & 0 & 10 & 20 & 30 & 40 & 50 & 60 & 70 \\
\hline$\overline{\mathrm{P} 1}$ & 5.81 & 10.60 & 10.05 & 7.31 & 6.25 & 6.05 & 5.98 & 5.84 \\
\hline $\mathrm{P} 2$ & 5.82 & 10.67 & 10.36 & 10.08 & 9.60 & 8.60 & 6.50 & 5.87 \\
\hline P3 & 5.58 & 10.65 & 8.92 & 6.80 & 6.64 & 6.01 & 5.90 & 5.56 \\
\hline $\mathrm{P} 4$ & 5.67 & 10.65 & 10.40 & 8.70 & 6.34 & 6.05 & 6.32 & 5.84 \\
\hline P5 & 5.81 & 10.70 & 9.80 & 9.40 & 9.00 & 7.90 & 6.50 & 5.90 \\
\hline P6 & 5.77 & 10.64 & 10.31 & 9.68 & 8.25 & 7.80 & 6.80 & 6.02 \\
\hline P7 & 5.81 & 10.70 & 9.80 & 9.40 & 9.00 & 7.90 & 6.50 & 5.90 \\
\hline P8 & 5.77 & 10.64 & 10.31 & 9.68 & 8.25 & 7.80 & 6.80 & 6.02 \\
\hline
\end{tabular}

Table 4b. Influence of pellets on the $\mathrm{pH}$ of solution.

\begin{tabular}{|c|c|c|c|c|c|c|c|c|}
\hline \multirow[b]{2}{*}{ Sample } & \multicolumn{8}{|c|}{$\mathrm{pH} /$ time $(\mathrm{min})$ in buffer $(\mathrm{pH}=6.8)$} \\
\hline & 0 & 10 & 20 & 30 & 40 & 50 & 60 & 70 \\
\hline $\mathrm{P} 1$ & 6.8 & 7.83 & 7.81 & 7.76 & 7.74 & 7.71 & 6.95 & 6.83 \\
\hline P2 & 6.8 & 7.50 & 7.32 & 7.13 & 7.01 & 6.92 & 6.89 & 6.83 \\
\hline P3 & 6.8 & 7.80 & 6.93 & 6.85 & 6.84 & 6.83 & 6.81 & 6.81 \\
\hline $\mathrm{P} 4$ & 6.8 & 7.59 & 7.22 & 6.93 & 6.90 & 6.86 & 6.84 & 6.81 \\
\hline P5 & 6.8 & 7.70 & 7.52 & 7.41 & 7.21 & 7.00 & 6.86 & 6.83 \\
\hline P6 & 6.8 & 7.71 & 7.58 & 7.45 & 7.21 & 7.02 & 6.90 & 6.86 \\
\hline P7 & 6.8 & 7.65 & 7.18 & 7.03 & 7.00 & 6.97 & 6.90 & 6.86 \\
\hline P8 & 6.8 & 7.68 & 7.41 & 7.32 & 7.20 & 7.00 & 6.87 & 6.85 \\
\hline
\end{tabular}




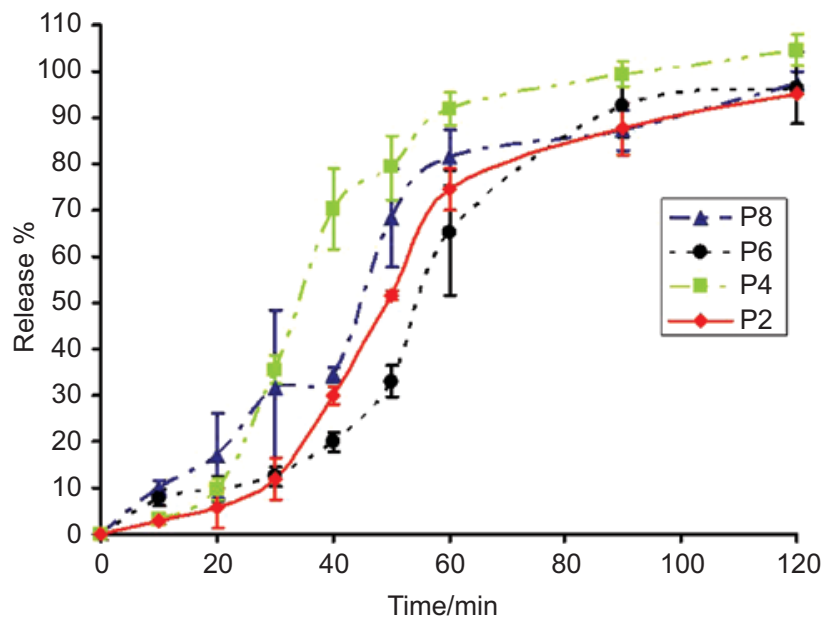

Figure 11. Dissolution profiles of the pellets containing trisodium phosphate. (See colour version of this figure online at www. informahealthcare.com/phd)

anhydrous and trisodium phosphate dodecahydrate) were used. The aim of the present study was to investigate the influence of these alkalizing components and the quantity and the composition of the wetting liquid on the formulation and dissolution of the pellets. It can be concluded that the shape parameters of the pellets were best when trisodium phosphate was the alkalizing component. As concerns the wetting liquid, the higher amount of water alone resulted in pellets with the best compact texture, with a smooth surface and considerable hardness. MCC formed a coherent gel-like network and the water-soluble trisodium phosphate underwent rapid recrystallization, resulting in strong solid bridges with the other particles. It is clear that the alkalizing component is able to increase the $\mathrm{pH}$ in the micromilieu. This effect is presumably manifested in the intestine too. In the in vitro experiments, the dissolution release complied with the texture of the pellets and the effect of $\mathrm{pH}$. The most uniform and total release of Atenolol was observed for sample P2.

In view of the good bioavailability, in vivo experiments will be carried out. The final dosage form, suitable for in vivo experiments, should be a preparation coated with a gastric-resistant polymer. The present results indicate that the final dosage form should involve pellet sample P2. This has suitable shape and very good hardness for the coating process, and the dissolution of Atenolol was appropriate.

\section{Declaration of interest}

The authors report no conflicts of interest. The authors alone are responsible for the content and writing of the paper.

\section{References}

1. Ghebre-Sellassie I. Pharmaceutical pelletisation technology. New York: Marcel Dekker Inc; 1989.
2. Otsuka M, Gao J, Matsuda Y. Effect of amount of added water during extrusion-spheronization process on pharmaceutical properties of granules. Drug Dev Ind Pharm 1994;20:2977-2992.

3. Newton JM. Extrusion/spheronization. In: Chulia D, Deleuil M, Pourcelot Y, editors. Powder technology and pharmaceutical processes. Amsterdam: Elsevier; 1994.

4. Noche C, Barochez BH, Brossard C, Horvath S, Cuiné A. Optimising the manufacturing process for controlled release pellets. Pharm Techn Eur 1994;4:39-46.

5. Fekete R. Effect of the formulation parameters on the characteristics of pellets. Drug Dev Ind Pharm 1998;24:1073-1076.

6. Santos H, Veiga F, Pina M, Podczeck F, Sousa J. Physical properties of chitosan pellets produced by extrusion/ spheronization: Influence of formulation variables. Int J Pharm 2002;246:153-169.

7. De Brabander C, Vervaet C, Remon JP. Development and evaluation of sustained release mini-matrices prepared via hot melt extrusion. J Control Release 2003;89:235-247.

8. De Brabander C, Vervaet C, Van Bortel L, Remon JP. Bioavailability of ibuprofen from hot-melt extruded mini-matrices. Int J Pharm 2004;271:77-84.

9. Nikam AP, Bodhankar SL, Piplani P, Bansal J, Thakurdesai PA. Betaadrenoreceptor blocking and antihypertensive activity of pp-24 a newly synthesized aryloxypropanolamie derivative. J Pharm Pharmcol 2008;60:1501-1506.

10. Niebyl JR. Teratology and drugs in pregnancy and lactation. In: Scott T, editor. Danforth's obstetrics and gynecology. 6th ed. Philadelphia: Lipincott; 1990.

11. Briggs GG, Freeman RK, Yaffe SJ. Drugs in pregnancy and lactation. 4th ed. Baltimore: Williams and Wilkins; 1994.

12. Tabacova SA, Kimmel CA. Atenolol: Pharmacokinetic/dynamic aspects of comparative developmental toxicity. Reprod Toxicol 2002;16:1-7.

13. Reeves PR, et al. Disposition and metabolism of atenolol in animals. Xenobiotica 1978;8:305-311.

14. Fitzgerald JD. The biological and clinical effects of atenolol (Tenormin), a cardioselective-antagonist. In: Goldberg ME, editor. Pharmacological and biochemical properties of drug substances. Vol. II. Washington DC: American Pharmaceutical Association Press; 1979.

15. Thompson FD. Review of clinical pharmacology of atenolol. Proc Royal Soc Med (Suppl. 5):1977;70:5-8.

16. Wallace JW. Cellulose derivatives and natural products utilized in pharmaceutics. In: Swarbrick J, Boylan JC, editors. Encyclopedia of pharmaceutical technology. Vol. 2. New York: Marcel Dekker; 1990:319-339.

17. Iyer RM, Augsburger LL, Parikh DM. Evaluation of drug layering and coating: Effect of process mode and binder level. Drug Dev Ind Pharm 1993;19:981-998.

18. Ye Z-W, Rombout P, Remon JP, Vervaet C, Van Den Mooter G. Correlationbetween the permeability of metoprololtartratethrough plasticized isolated ethylcellulose/hydroxypropylmethylcellulose films and drug release from reservoir pellets. Eur J Pharm Biopharm 2007;67:485-490.

19. Fan TY, Wei SL, Yan WW, Chen DB, Li J. An investigation of pulsatile release tablets with ethylcellulose and Eudragit L as film coating materials and cross-linked polyvinylpyrrolidone in the core tablets. J Control Release 2001;77:245-251.

20. Huang J, Wigent RJ, Bentzley CM, Schwartz JB. Nifedipine solid dispersion in microparticles of ammonio methacrylate copolymer and ethylcellulose binary blend for controlled drug delivery. Effect of drug loading on release kinetics. Int J Pharm 2006;319:44-54.

21. Corti G, Cirri M, Maestrelli F, Mennini N, Mura P. Sustainedrelease matrix tablets of metformin hydrochloride in combination with triacetyl- $\beta$-cyclodextrin. Eur J Pharm Biopharm 2008;68:303-309.

22. Shah RD, Kabadi M, Pope DG, Augsburger LL. Physicomechanical characterization of the extrusion-spheronization process. 


\section{E. I. Hamedelniel et al.}

2. Rheological determinants for successful extrusion and spheronisation. Pharm Res 1995;12:496-507.

23. Newton JM. Extrusion and extruders. In: Swarbrick J, Boylan JC, editors. Encyclopedia of pharmaceutical technology. New York and Basel: Marcel Dekker Inc; 2002: 1220-1236.
24. Hamedelniel EI, Bajdik J, Pintye-Hódi, K. Optimization of preparation of matrix pellets containing ethylcellulose. Chem Eng Process 2010;49:120-124.

25. Nürnberg E, Surmann P, editors. Hagers Handbuch der pharmazeutischen Praxis. 5th ed. Methoden, Berlin, New York: Springer Verlag, Bd. 2; 1991. p 60. 
IV. 


\title{
Delayed release matrix pellet preparation containing an alkalizing pore-former agent
}

\author{
Elnazeer I. Hamedelniel, János Bajdik, Tamás Sovány, Klára Pintye-Hódi* \\ Department of Pharmaceutical Technology, University of Szeged, Eötvös u. 6, H-6720 Szeged, Hungary
}

\begin{abstract}
A B S T R A C T
The aim of this study was to develop a delayed-release matrix pellet containing atenolol as active pharmaceutical ingredient. The matrix additionally contained trisodium phosphate dodecahydrate as alkalizing pore-former agent to enhance the dissolution of the atenolol at pH 6.8. The delayed release was ensured by coating with a gastroresistant polymer. For this purpose, an acryl EZE MP aqueous dispersion was used, which is suggested in the literature for pellet coating. Before this functional film coating, a protective polymer layer was developed, to prevent direct contact between the alkalizing layer and the acryl EZE. The results of in vitro dissolution tests demonstrated that the double-coated pellet preparation is a delayed-release solid dosage form.
\end{abstract}

( 2010 The Institution of Chemical Engineers. Published by Elsevier B.V. All rights reserved.

Keywords: Matrix pellet; Pore-former agent; Coating; Dissolution test; Delayed release

\section{Introduction}

Pellets are frequently coated in order to achieve sustained drug release or to deliver a drug to the specific absorption site in the gastrointestinal tract (e.g. enteric-coated or colon-targeted drug delivery) (Ghebre-Sellassie, 1989).

Enteric-coated pellets as dosage forms are especially suited for the administration of drugs which are not stable in the gastric fluids or which can cause irritation of the gastric mucosa and which are absorbed in the duodenum or upper intestine (Erkoboni, 1997).

The incorporation of microcrystalline cellulose (MCC) is necessary for successful extrusion and spheronization, (Shah et al., 1995) in many formulations, since it provides the wetted mass with the appropriate rheological properties (Newton, 2002). In order to obtain a very good mass for this process, it is necessary to use MCC and the matrix former together. Ethylcellulose (EC) has frequently been used as a coating material for tablets and pellets (Wallace, 1990; Iyer et al., 1993; Ye et al., 2007) and as a binder in the preparation of microcapsules and microspheres, but it is also used as a monolithic matrix former for formulations in which modified drug release is ensured by diffusion (Corti et al., 2008; Huang et al., 2006; Fan et al., 2001). Several commercially available polymers are suitable for the coating of pharmaceutical dosage forms (McGinity,
1997), and some can be used to control the drug release kinetics. However, it is often difficult to adjust a particular release profile to the pharmacokinetic characteristics of the drug. Different formulation and processing parameters can be varied in order to optimize the drug release patterns, e.g. coating level, type polymers, etc., but these variations are often restricted because reasonable film properties must be provided and production on a large scale must be feasible. To overcome these restrictions, polymer blends can be used as coating materials controlling drug release (Dashevsky et al., 2004; Lecomte et al., 2004; Khan et al., 1999).

The composition of the film coating and the nature of the coating technique (the use of aqueous dispersions or organic solutions) can affect the properties of the resulting polymeric membranes, including their permeability for water and drug, mechanical resistance and dissolution behaviour (Garcia-Arieta et al., 1996; Lorck et al., 1997; Wesseling and Bodmeier, 1999). Atenolol (At) was used as active pharmaceutical ingredient. It is a potent cardioselective $\beta$-adrenoreceptor blocking agent (Nikam et al., 2008) applied for the treatment of hypertension, including hypertension in pregnancy (Niebyl, 1990; Briggs et al., 1994). Following oral administration, the absorption of the drug in humans and most laboratory animal species is rapid, but incomplete (50-60\%). At is well absorbed at $\mathrm{pH}>7.5$ (Tabacova and Kimmel, 2002), especially in the dog,

\footnotetext{
* Corresponding author. Tel.: +36 62545576; fax: +36 62545571.

E-mail address: klara.hodi@pharm.u-szeged.hu (K. Pintye-Hódi).

Received 22 July 2010; Received in revised form 16 November 2010; Accepted 1 December 2010

0263-8762/\$ - see front matter @ 2010 The Institution of Chemical Engineers. Published by Elsevier B.V. All rights reserved.

doi:10.1016/j.cherd.2010.12.003
} 
in which the $\mathrm{pH}$ of the ileum is $>8$, and in which absorption from the gut is therefore almost complete: $98 \%$ of the total dose (Reeves et al., 1978). This striking difference in intestinal absorption has been attributed to interspecies differences in intestinal luminal $\mathrm{pH}$. There is no evidence of an alternative biliary route for excretion in either humans or experimental animals (Fitzgerald, 1979; Thompson, 1977). As At is well absorbed at pH $>7.5$ (Tabacova and Kimmel, 2002), it must be administered with a component that ensures a suitable local $\mathrm{pH}$. Preparation of a multiparticulate matrix system with an alkalizing component is a reasonable way to ensure an appropriately alkaline micromilieu (Hamedelniel et al., 2010a). Trisodium phosphate dodecahydrate was incorporated as alkalizing and pore-former substance; our previous work had revealed that a matrix-former polymer is required to control the parallel release of At and the alkalizing component utilized (Hamedelniel et al., 2010b) and to ensure that the dosage form reaches the intestine in intact form. Thus, it is necessary to prepare a delayed-release product. For this purpose, various polymers, are available, such as the generally accepted enteric polymer Eudragit L100-5 $5^{\circledR}$, which is soluble from $\mathrm{pH} 6.0$ due to hydration of the ionized carboxylate groups (Moustafine et al., 2005). There are organic solvent-based systems and aqueous dispersions. The aqueous coating systems have numerous advantages over the organic solvent-based systems, for example with respect to ecological, toxicological and manufacturing safety concerns. However, the major limitation of many aqueous enteric coating formulations is the risk of premature drug release (permeation) through the enteric coat in the stomach. This can be due to an increased permeability of aqueous film coatings (Guo et al., 2002; Chang, 1990) or to a high water-solubility of the drug (Bianchini et al., 1991). Subcoating materials have been widely used in combination with enteric polymers to promote adhesion of the functional polymer (Obara et al., 1999), to function as a moisture barrier (Felton et al., 1995), and to prevent interactions between an active pharmaceutical ingredient and an enteric coating (Bozdag et al., 1999). Other researchers have described an increased gastric resistance of enteric-coated dosage forms in the presence of a polymeric subcoat (Crotts et al., 2001; Guo et al., 2002; Bruce et al., 2003). Our formula contained a high amount of a strong alkalizing agent, which influenced the dissolution of the At earlier than required. Hence, it was necessary to separate the core from the functional coating layer.

The aim of the present work was to study the delayed effects of matrix pellets made with a gastric acid-resistant polymer on the release of At from pellets containing an alkalizing pore-former agent.

\section{Materials and methods}

\subsection{Materials}

At (Ariane Organochem Private Ltd., Mumbai, India) was applied as model drug, and EC (Ethocel standard 10 premium, Colorcon Ltd. Dartford, England) and MCC (Vivapur 103, Rettenmaier \& Söhne $\mathrm{GmbH}$, Rosenberg, Germany) as pharmaceutical excipients. $\mathrm{Na}_{3} \mathrm{PO}_{4} \cdot 12 \mathrm{H}_{2} \mathrm{O}$ (VWR International, Belgium) was used as alkalizing agent and purified water served as wetting component. Opadry clear (hydroxyl propyl methylcellulose (HPMC)) and acryl EZE MP (Colorcon Ltd., Dartford, England) were utilized as coating dispersions. The latter contains methacrylic acid copolymer (Eudragit L100-55 ${ }^{\circledR}$ ) as enteric coated material and plasticizer, which are necessary during coating. Dimethicone (Silfar E 1049, Wacker Chemie AG, supplier: Brenntag Hungaria Kereskedelmi Kft, Hungary) was used as anti-foaming agent, and Ariavit sunset yellow CI 15985 (Sensient Food Colors Hungary Kft, Hungary) as dye.

\subsection{Preparation of pellets}

$150 \mathrm{~g}$ of powder mixture was prepared from $37.5 \mathrm{~g}$ of At, $37.5 \mathrm{~g}$ of sodium phosphate, $45 \mathrm{~g}$ of MCC and $30 \mathrm{~g}$ of EC 10 . To obtain a uniform mixture, the powder was blended at $50 \mathrm{rpm}$ for $10 \mathrm{~min}$ with a Turbula mixer (W.A. Bachofen, Basel, Switzerland).

Wetting was performed in a high-shear mixer (ProCepT 4M8 granulator, ProCepT nv, Zelzate, Belgium), for kneading by the addition of water as granulating liquid. The kneading parameters were as follows: impeller speed $1500 \mathrm{rpm}$, chopper speed $2000 \mathrm{rpm}$, and dosing speed $5 \mathrm{ml} / \mathrm{min}$.

The wet mass obtained was extruded by a mini screw extruder (Caleva Ltd., Sturminster Newton, Dorset, UK) equipped with an axial screen with dies $1 \mathrm{~mm}$ in diameter and $4 \mathrm{~mm}$ in length, operating at $90 \mathrm{rpm}$. The jacked barrel of the extruder was cooled by water at $25 \pm 2{ }^{\circ} \mathrm{C}$. Each extrudate was collected in a container before it was spheronized. About $40 \mathrm{~g}$ of extrudate was spheronized at a time, on a spheronizer $12 \mathrm{~cm}$ in diameter (Model-120, G.B. Caleva Ltd., Sturminster Newton, Dorset, UK) fitted with a cross-hatch grooved plate, for $10 \mathrm{~min}$ at $1000 \mathrm{rpm}$. The pellets were dried under the same conditions, at $40 \pm 2{ }^{\circ} \mathrm{C}$ for $24 \mathrm{~h}$.

\subsection{Morphological study}

The particle size and the shape of the pellet surface before and after coating were studied by using a system consisting of a stereomicroscope (Zeiss Stemi 2000-C, Carl Zeiss GmbH, Vienna, Austria) and a ring light with a cold light source (Leica KL 1500, Cambridge, UK). A Quantimet 500 (Q500MC) image processing and analysis system (Leica Cambridge Ltd., Cambridge, UK) was used. The aspect ratio was utilized for the evaluation of the shape of the particles.

The surfaces of various samples and the internal structures (cross-sections) of the pellets were tested with a scanning electron microscope (SEM) (Hitachi S4700, Hitachi Scientific Instruments Ltd., Tokyo, Japan). A SEM sputter coating unit (Polaron E5100, VG Microtech, UK) was used to charge the surfaces for the SEM measurements. The air pressure was 1.3-13 $\mathrm{mPa}$.

\subsection{Mechanical properties of the pellets}

The breaking strength was tested for pellets with size fractions of $1500-2000 \mu \mathrm{m}$ in diameter. The strength tester and the software were developed in our institute. The tester contains a special specimen holder and a jowl, and is connected to a computer via an interface. Thus, the ultimate deformation force can be measured, and the process (force-time and force-displacement curves) can also be followed. The specimen is located horizontally on a special plate and the jowl moves vertically. Twenty parallel measurements were performed.

The measurement range of the force was $0-200 \mathrm{~N}$, the speed of the jowl was $20 \mathrm{~mm} / \mathrm{min}$, and the output was $0-5 \mathrm{~V}$. The sensor was a Unicell force measuring instrument, calibrated with the C9B $200 \mathrm{~N}$ cell. 


\subsection{Coating of pellets}

\subsubsection{Preparation of coating fluids}

2.5.1.1. Opadry solution for subcoating. $600 \mathrm{ml}$ of distilled water was stirred at $400 \mathrm{rpm}$ for $5 \mathrm{~min}$, during which $5 \%$ of Opadry clear was added in portions, and the resulting mixture was shaken gently and stirred at $200 \mathrm{rpm}$ for $1 \mathrm{~h}$, and then filtered.

2.5.1.2. Acryl EZE MP dispersion liquid for coating. $120 \mathrm{~g}$ of acryl EZE MP and $1 \mathrm{~g}$ of Dimethicone were weighed and added in portions to $479 \mathrm{~g}$ of distilled water in a beaker during stirring at $400 \mathrm{rpm}$ for at least $2 \mathrm{~h}$, and the dispersed liquid was then passed through a $0.25 \mathrm{~mm}$ sieve to obtain a dispersion system with uniform particle size.

\subsubsection{Coating process}

$200 \mathrm{~g}$ of pellet cores was coated in a fluidized bed coater equipped with a Wurster column (Strea 1; Aeromatic-Fielder, Bubendorf, Switzerland) The process parameters were as follows: inlet temperature $40 \pm 2{ }^{\circ} \mathrm{C}$, product temperature $50 \pm 2^{\circ} \mathrm{C}$, spray rate $1.5-3 \mathrm{~g} / \mathrm{min}$, atomization pressure 2 bar, nozzle diameter $1 \mathrm{~mm}$, and air volume $95 \mathrm{~m}^{3} / \mathrm{h}$. After coating, the beads were further fluidized for $10 \mathrm{~min}$ and subsequently cured in an oven for $24 \mathrm{~h}$ at $40^{\circ} \mathrm{C}$.

\subsection{Dissolution tests}

$100 \mathrm{mg}$ of pellets was filled into HPMC (hydroxyl propyl methylcellusose) capsules, which were placed into the basket of a dissolution tester (Erweka DT 700, Heusenstamm, Germany). The rotational speed of the baskets was set at $100 \mathrm{rpm}$. The drug release from the pellets was analysed at $37.0 \pm 0.5^{\circ} \mathrm{C}$. The dissolution system was combined with an automatic sampling station. Dissolution studies $(n=3)$ were carried out in $900 \mathrm{ml}$ of $\mathrm{HCl} / \mathrm{NaCl}$ solution ( $\mathrm{pH}$ 1.2). After $2 \mathrm{~h}$, the $\mathrm{pH}$ of the medium was changed from 1.2 to 6.8 to simulate the gastric transition, according to the European Pharmacopoeia (2008). Samples of $5 \mathrm{ml}$ were withdrawn at 10 , $30,60,90$ and $120 \mathrm{~min}$ from the $\mathrm{HCl}$ solution at $\mathrm{pH} 1.2$ and at 10 , $20,30,40,50,60,90,120$ and $180 \mathrm{~min}$ from the phosphate buffer at $\mathrm{pH}$ 6.8. The content of At was measured spectrophotometrically (Unicam He(ios Alpha, Spectronic Unicam, Cambridge, $\mathrm{UK}$ ) at $\lambda_{\max }=202 \mathrm{~nm}(\mathrm{HCl} / \mathrm{NaCl} \mathrm{pH} \mathrm{1.2)} \mathrm{and} 224 \mathrm{~nm}$ (phosphate buffer $\mathrm{pH}$ 6.8).

\section{Results and discussion}

The hardness of the pellet was found to be $12.29 \pm 2.03 \mathrm{~N}$, which is very good for coating. The alkalizing component could promote the wettability of the powder mixture. This effect improved the plasticity of the wetted mass, enhanced the spheronization, and determined not only the mechanical properties, but also the shape of the pellets. During drying, rather fast recrystallization of the trisodium phosphate

\section{Table 1 - Shape parameters of the pellet.}

\begin{tabular}{llll} 
Sample & $\begin{array}{l}\text { Breadth } \\
(\mu \mathrm{m})\end{array}$ & $\begin{array}{l}\text { Length } \\
(\mu \mathrm{m})\end{array}$ & Aspect ratio \\
\hline Before coating & $1776 \pm 139$ & $1954 \pm 148$ & $1.16 \pm 0.102$ \\
After subcoating & $1806 \pm 111$ & $1990 \pm 139$ & $1.09 \pm 0.07$ \\
After double coating & $1876 \pm 54$ & $2065 \pm 58$ & $1.08 \pm 0.06$ \\
\hline
\end{tabular}

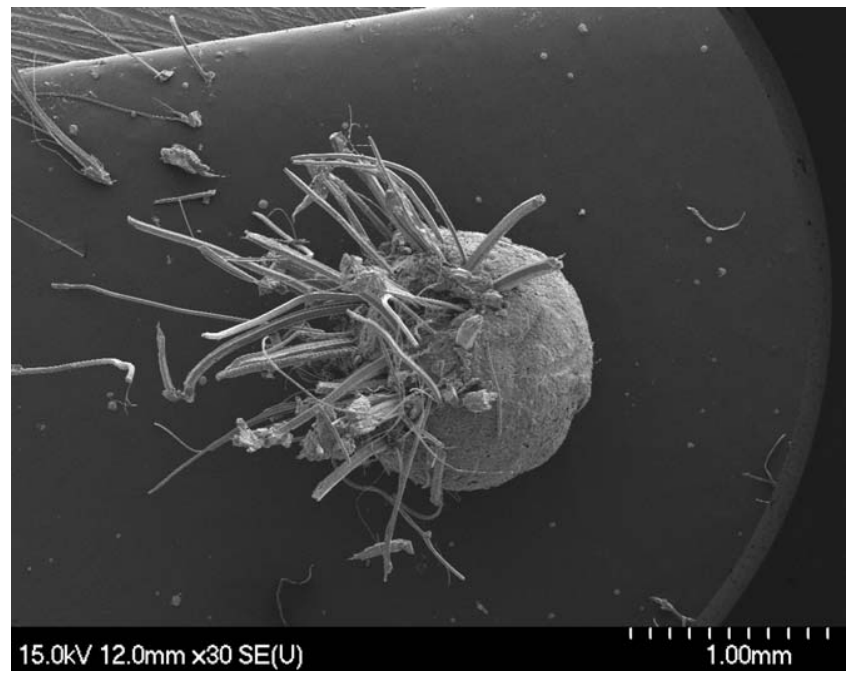

Fig. 1 - Trisodium phosphate crystals on the surface of the uncoated pellet.

occurred, resulting in strong solid bridges with the MCC particles. Table 1 reveals that the pellets before coating were close to spherical, with an aspect ratio close to 1 . This means that this sample is very good for coating. It can be seen from the data that the shape of the pellets after coating was more rounded. This means that they were smoothed by the covering layer of the coating polymer.

On SEM investigation, some needle crystals was observed on the surface of the uncoated pellets (Fig. 1). These crystals developed through the recrystallization of trisodium phosphate during drying, which can break down easily from the surface and lead to pore formation on the surface. This is the reason for the higher dissolution (about $30 \%$ ) in gastric juice in spite of the gastric juice-resistant coating (Aryl EZE MP, mass $17 \%, w / w)$ (Fig. 2). The alkalizing component has very good water solubility and can dissolve in the water component of the coating dispersion, so that some of it can migrate into the polymer film. Accordingly, it was necessary to protect the core before the functional coating. The protective layer used was HPMC (Opadry clear) (mass 3\%,w/w). After drying of the protective layer (10 $\mathrm{min}$ ), the coating was continued with the functional, pH-dependent polymer dispersion (acryl EZE MP).

The dissolution test revealed that At could not dissolve from the double-coated pellets in gastric juice because the protective layer closed the pores of the core and did not allow the migration of any component in the outer layer. However, the total amount of drug could dissolve in the phosphate buffer $(\mathrm{pH}=6.8)$ during $3 \mathrm{~h}$ (Fig. 3). The dissolution exhibited a typi-

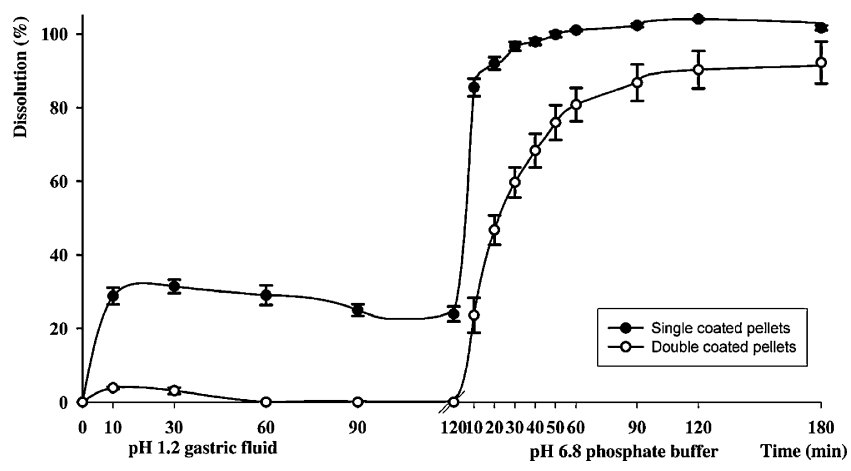

Fig. 2 - Dissolution profiles of atenolol from the single-coated and double-coated pellets. 


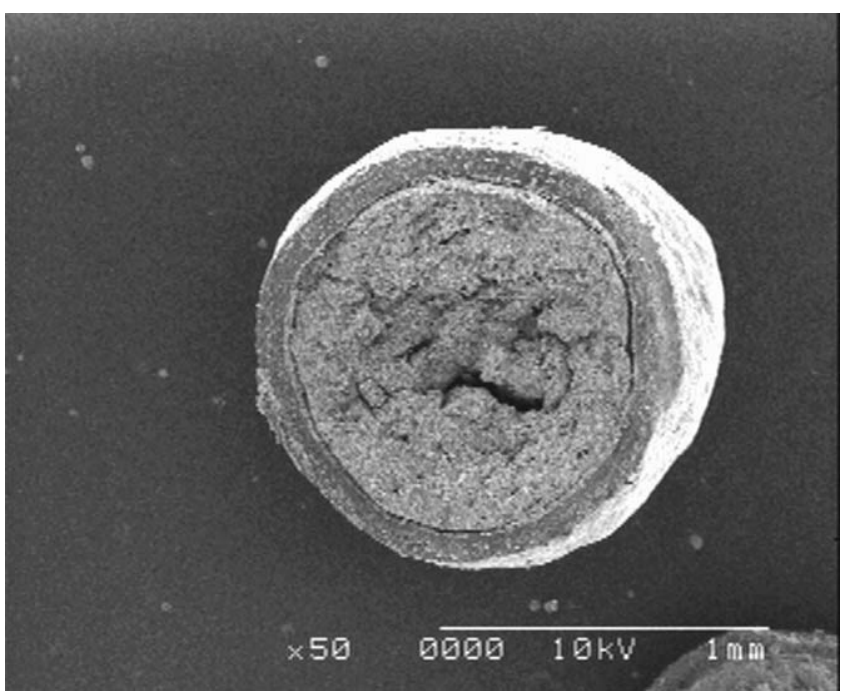

Fig. 3 - Cross-section of the double-coated pellet (SEM). Magn.: $50 x$.

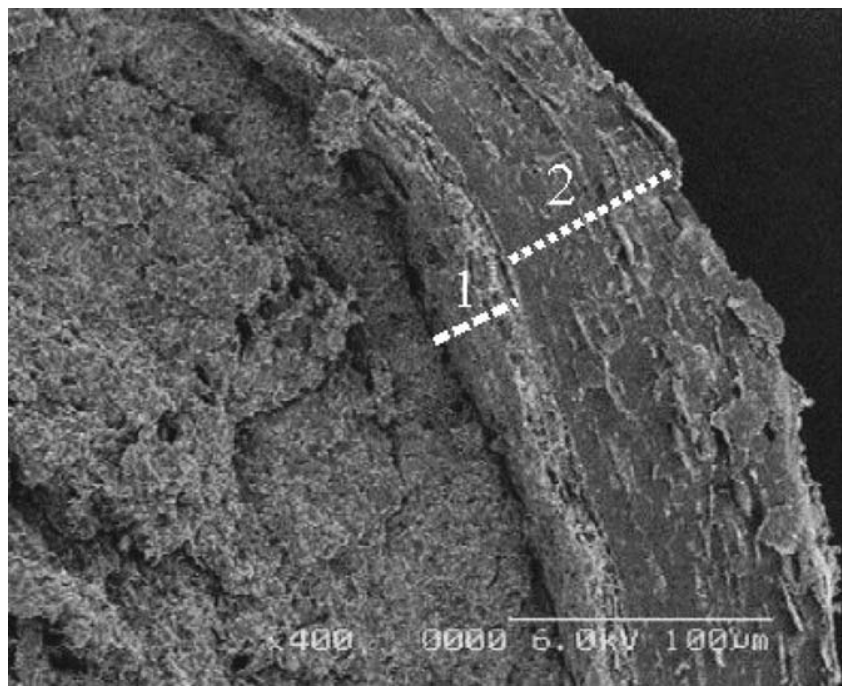

Fig. 4 - Cross-section of the double-coated pellet (SEM). Magn.: 400x. (1) Protective layer; (2) functional layer.

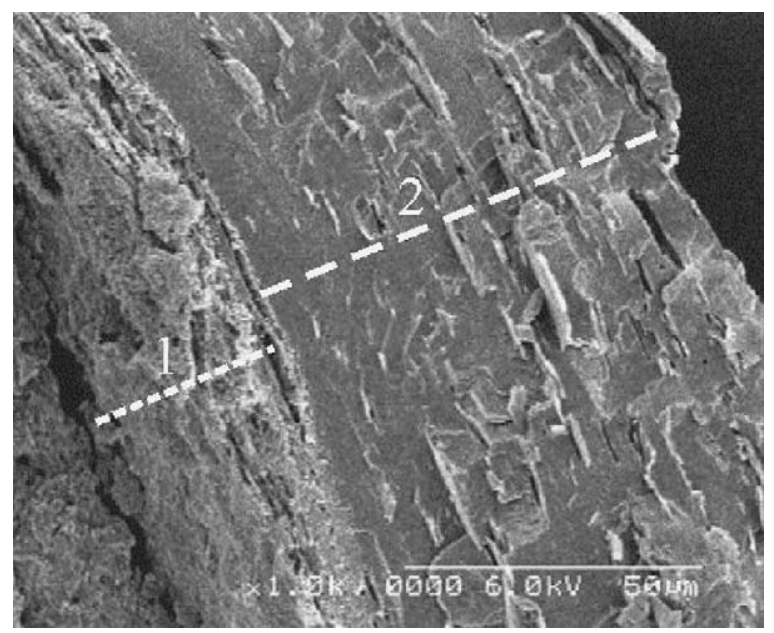

Fig. 5 - Cross-section of the double-coated pellet (SEM). Magn.: $1000 x$. (1) Protective layer; (2) functional layer. cal delayed-release profile: the dissolution of the drug started only after $2 \mathrm{~h}$. This means that the protecting layer on the surface of the core was working efficiently. The aqueous solution could not pass into the core and the alkalizing component could not migrate into the acryl EZE film. In the phosphate buffer, both coating layers and hence the alkalizing component could dissolve, and the alkalizing micromilieu enhanced the dissolution of At from the matrix pellet.

Figs. 3-5 illustrate the cross-section of the double-coated pellet. At low magnification (Fig. 3), the spherical form and a relative large pore in the middle of the pellet can be observed, but around the pore the matrix is rather compact. At higher magnification (Fig. 4), the coating layer is clearly visible and the two polymer layers are distinguishable (Fig. 5); it can be supposed that the adherence between the two kinds of polymer is very strong.

\section{Conclusions}

The results of the experiments revealed that the pellets prepared by extrusion and spheronization were spherical and had high strength. This product was suitable for coating. The in vitro dissolution tests demonstrated that the alkalizing component promoted the dissolution of the total amount of At from the pellets at $\mathrm{pH} 6.8$, but use of a protective polymer layer was necessary before the functional polymer coating. This double-coated pellet is an excellent product which is suitable for filling into capsules.

\section{Acknowledgement}

The authors wish to express their gratitude to Colorcon for the supply of polymers.

\section{References}

Bianchini, R., Resciniti, M., Vecchio, C., 1991. Technology evaluation of aqueous enteric coating systems with and without insoluble additives. Drug Dev. Ind. Pharm. 17, 1779-1794.

Bozdag, S., Calis, S., Sumnu, M., 1999. Formulation and stability evaluation of enteric coated omeprazole formulations. STP Pharma Sci. 9, 321-327.

Briggs, G.G., Freeman, R.K., Yaffe, S.J., 1994. Drugs in Pregnancy and Lactation, 4th ed. Williams and Wilkins, Baltimore.

Bruce, L.D., Koleng, J.J., McGinity, J.W., 2003. The influence of polymeric subcoats and pellet formulation on the release of chlorpheniramine maleate fromenteric coated pellets. Drug Dev. Ind. Pharm. 29, 909-924.

Chang, R.K., 1990. A comparison of rheological and enteric properties among organic solutions, ammonium salt aqueous solutions, and latex systems of some enteric polymers. Pharm. Technol. 10, 62-70.

Corti, G., Cirri, M., Maestrelli, F., Mennini, N., Mura, P., 2008. Sustained-release matrix tablets of metformin hydrochloride in combination with triacetyl- $\beta$-cyclodextrin. Eur. J. Pharm. Biopharm. 68, 303-309.

Crotts, G., Sheth, A., Twist, J., Ghebre-Sellassie, I., 2001. Development of an enteric coating formulation and process for tablets primarily composed of a highly water-soluble, organic acid. Eur. J. Pharm. Biopharm. 51, 71-76.

Dashevsky, A., Kolter, K., Bodmeier, R., 2004. pH-independent release of a basic drug from pellets coated with the extended release polymer dispersion Kollicoat SR $30 \mathrm{D}$ and the enteric polymer dispersion Kollicoat MAE 30 DP. Eur. J. Pharm. Biopharm. 58, 45-49. 
Erkoboni, K.A., 1997. Extrusion/spheronisation. In: Parikh, D. (Ed.), Handbook of Pharmaceutical Technology. Marcel Dekker Inc., New York.

European Pharmacopoeia 6.0, 2008. Dissolution test for solid dosage forms (Chapter 2.9.3). In: European Pharmacopoeia 6.0. Deutscher Apotheker Verlag, Stuttgart.

Fan, T.Y., Wei, S.L., Yan, W.W., Chen, D.B., Li, J., 2001. An investigation of pulsatile release tablets with ethylcellulose and Eudragit $\mathrm{L}$ as film coating materials and cross-linked polyvinylpyrrolidone in the core tablets. J. Control. Release 77, 245-251.

Felton, L.A., Haase, M.M., Shah, N.H., Zhang, G., Infeld, M.H., Malick, A.W., McGinity, J.W., 1995. Physical and enteric properties of soft gelatin capsules coated with Eudragit L 30 D-55. Int. J. Pharm. 113, 17-24.

Fitzgerald, J.D., 1979. The biological and clinical effects of atenolol (Tenormin), a cardioselective-antagonist. In: Goldberg, M.E. (Ed.), Pharmacological and Biochemical Properties of Drug Substances, Volume II. American Pharmaceutical Association Press, Washington, DC.

Garcia-Arieta, A., Torrado-Santiago, D., Torrado, J.J., 1996. Comparative study of aqueous and organic enteric coatings of chlorpheniramine maleate tablets. Drug Dev. Ind. Pharm. 22, 579-585.

Ghebre-Sellassie, I., 1989. Pellets: a general overview. In: Ghebre-Sellassie, I. (Ed.), Pharmaceutical Pelletization Technology. Marcel Dekker, New York, pp. 1-13.

Guo, H.X., Heina“ma“ki, J., Yliruusi, J., 2002. Amylopectin as a subcoating material improves the acidic resistance of enteric-coated pellets containing a freely soluble drug. Int. J. Pharm. 235, 79-86.

Hamedelniel, E.I., Bajdik, J., Pintye-Hódi, K., 2010a. Optimization of preparation of matrix pellets containing ethylcellulose. Chem. Eng. Process 49, 120-124.

Hamedelniel, E.I., Bajdik, J., Kása P., Pintye-Hódi, K., 2010b. Study of the influence of alkalizing components on matrix pellets prepared by extrusion/spheronization. Pharm. Dev. Technol., in press, doi:10.3109/10837450.2010.531734.

Huang, J., Wigent, R.J., Bentzley, C.M., Schwartz, J.B., 2006. Nifedipine solid dispersion in microparticles of ammonio methacrylate copolymer and ethylcellulose binary blend for controlled drug delivery, Effect of drug loading on release kinetics. Int. J. Pharm. 319, 44-54.

Iyer, R.M., Augsburger, L.L., Parikh, D.M., 1993. Evaluation of drug layering and coating: effect of process mode and binder level. Drug Dev. Ind. Pharm. 19, 981-998.

Khan, M.Z.I., Prebeg, Z., Kurjakovic, N., 1999. A pH-dependent colon targeted oral drug delivery system using methacrylic acid copolymers. I. Manipulation of drug release using Eudragit L100-55 and Eudragit S100 combinations. J. Control. Release 58, 215-222.

Lecomte, F., Siepmann, J., Walther, M., MacRae, R.J., Bodmeier, R., 2004. Polymer blends used for the coating of multiparticulates: comparison of aqueous and organic coating techniques. Pharm. Res. 21, 882-890.

Lorck, C.A., Grunenberg, P.C., Juenger, H., Laicher, A., 1997. Influence of process parameters on sustained-release theophylline pellets coated with aqueous polymer dispersions and organic solvent-based polymer solutions. Eur. J. Pharm. Biopharm. 43, 149-157.

McGinity, J.W., 1997. Aqueous Polymeric Coatings for Pharmaceutical Dosage Forms, 2nd ed. Marcel Dekker, New York.

Moustafine, R.I., Kabanova, T.V., Kemenova, V.A., Van den Mooter, G., 2005. Characteristics of interpolyelectrolyte complexes of Eudragit E100 with Eudragit L100. J. Control. Release 103, 191-198.

Newton, J.M., 2002. Extrusion and Extruders. In: Swarbrick, J., Boylan, J.C. (Eds.), Encyclopedia of Pharmaceutical Technology. Marcel Dekker, Inc., New York/Basel, pp. 1220-1236.

Niebyl, J.R., 1990. Teratology and drugs in pregnancy and lactation. In: Scott, T. (Ed.), Danforth's Obstetrics and Gynecology. , 6th ed. Lipincott, Philadelphia.

Nikam, A.P., Bodhankar, S.L., Piplani, P., Bansal, J., Thakurdesai, P.A., 2008. Beta-adrenoreceptor blocking and antihypertensive activity of pp-24 a newly synthesized aryloxypropanolamie derivative. J. Pharm. Pharmcol. 60, 1501-1506.

Obara, S., Maruyama, N., Nishiyama, Y., Kokubo, H., 1999. Dry coating: an innovative enteric coating method using a cellulose derivative. Eur. J. Pharm. Biopharm. 47, 51-59.

Reeves, P.R., Barnfield, D.J., Longshaw, S., et al., 1978. Disposition and metabolism of atenolol in animals. Xenobiotica 8, 305-311.

Shah, R.D., Kabadi, M., Pope, D.G., Augsburger, L.L., 1995. Physicomechanical characterization of the extrusion-spheronization process. 2. Rheological determinants for successful extrusion and spheronisation. Pharm. Res. 12, 496-507.

Tabacova, S.A., Kimmel, C.A., 2002. Atenolol: pharmacokinetic/dynamic aspects of comparative developmental toxicity. Reprod. Toxicol. 16, 1-7.

Thompson, F.D., 1977. Review of clinical pharmacology of atenolol. Proc. R. Soc. Med. 70 (Suppl. 5), 5-8.

Wallace, J.W., 1990. Cellulose derivatives and natural products utilized in pharmaceutics. In: Swarbrick, J., Boylan, J.C. (Eds.), Encyclopedia of Pharmaceutical Technology, vol. 2. Marcel Dekker, New York, pp. 319-339.

Wesseling, M., Bodmeier, R., 1999. Drug release from beads coated with an aqueous colloidal ethylcellulose dispersion, Aquacoat, or an organic ethylcellulose solution. Eur. J. Pharm. Biopharm. 47, 33-38.

Ye, Z.-W., Rombout, P., Remon, J.P., Vervaet, C., Van Den Mooter, G., 2007. Correlation between the permeability of metoprolol tartrate through plasticized isolated ethylcellulose/hydroxyl propyl methylcellulose films and drug release from reservoir pellets. Eur. J. Pharm. Biopharm. 67, 485-490. 